University of Louisville

ThinkIR: The University of Louisville's Institutional Repository

Electronic Theses and Dissertations

$12-2014$

\title{
Post-transcriptional regulation of the Type IIA sodium-phosphate cotransporter by parathyroid hormone.
}

Rebecca Dale Murray
University of Louisville

Follow this and additional works at: https://ir.library.louisville.edu/etd

Part of the Physiology Commons

\section{Recommended Citation}

Murray, Rebecca Dale, "Post-transcriptional regulation of the Type IIA sodium-phosphate cotransporter by parathyroid hormone." (2014). Electronic Theses and Dissertations. Paper 1749.

https://doi.org/10.18297/etd/1749

This Doctoral Dissertation is brought to you for free and open access by ThinkIR: The University of Louisville's Institutional Repository. It has been accepted for inclusion in Electronic Theses and Dissertations by an authorized administrator of ThinkIR: The University of Louisville's Institutional Repository. This title appears here courtesy of the author, who has retained all other copyrights. For more information, please contact thinkir@louisville.edu. 
POST-TRANSCRIPTIONAL REGULATION OF THE TYPE IIA SODIUMPHOSPHATE COTRANSPORTER BY PARATHYROID HORMONE

\author{
By \\ Rebecca Dale Murray \\ B.A., Northwestern University, 2009 \\ M.S., University of Louisville, 2013
}

\begin{abstract}
A Dissertation
Submitted to the Faculty of the School of Medicine of the University of Louisville in Partial Fulfillment of the Requirements for the Degree of
\end{abstract}

Doctor of Philosophy

Department of Physiology \& Biophysics

University of Louisville Louisville, KY

December 2014 
Copyright 2014 by Rebecca Dale Murray

All rights reserved 

POST-TRANSCRIPTIONAL REGULATION OF THE TYPE IIA SODIUMPHOSPHATE COTRANSPORTER BY PARATHYROID HORMONE

$$
\text { By }
$$

Rebecca Dale Murray

A Dissertation Approved on

November 24, 2014

by the following Dissertation Committee:

Eleanor D. Lederer, M.D.

Syed J. Khundmiri, Ph.D.

Barbara J. Clark, Ph.D.

Cynthia J. Miller, Ph.D.

Jeff C. Falcone, Ph.D.

Irving G. Joshua, Ph.D. 


\section{DEDICATION}

This dissertation is dedicated to my parents,

Paul and Meridith Murray,

for pancakes and applesauce at the Mariann. 


\section{ACKNOWLEDGEMENTS}

The work within this dissertation represents the culmination of several years of training, during which time I have been extremely fortunate to receive help from many talented and supportive individuals. I would like to thank Nina Lesousky, Sarah Salyer, and Francesca Kingery for helping me to find my feet in the lab. I would also like to thank my committee members for their support and sage advice, and in particular I would like to thank Dr. Clark for committing to a multi-year collaboration with a graduate student. I am very much indebted to Jalal for his constant guidance and support, and for putting up with a very sassy graduate student these past four years. Lastly I would like to thank Dr. Lederer, not only for agreeing to mentor a graduate student with no prior research experience, but for providing me with an outstanding predoctoral training full of

intellectual challenges, well-timed humor, and endless support of my current and future endeavors. 


\title{
ABSTRACT \\ POST-TRANSCRIPTION REGULATION OF THE TYPE IIA SODIUM-PHOSPHATE COTRANSPORTER BY PARATHYROID HORMONE
}

\author{
Rebecca D. Murray
}

November 24, 2014

The type IIa sodium-phosphate cotransporter (Npt2a) is expressed in the apical membrane of the renal proximal tubule and is responsible for the reabsorption of the majority of the filtered load of phosphate. Parathyroid hormone (PTH) is secreted by the parathyroid gland in response to a decrease in serum calcium or an increase in serum phosphorus, and acutely induces phosphaturia through the rapid stimulation of endocytosis of Npt2a and its subsequent lysosomal degradation. Chronic PTH stimulation leads to sustained phosphaturia, but the mechanisms for the chronic regulation of Npt2a by PTH remain unclear. We hypothesize that PTH decreases Npt2a mRNA levels as a mechanism for inducing chronic phosphaturia. We address this hypothesis within three specific aims. The first specific aim addresses the kinetics and mechanisms of the PTHstimulated decrease in Npt2a mRNA expression. The second aim details the signaling pathways involved in PTH-mediated downregulation of Npt2a mRNA. Lastly, the third aim characterizes the PTH-responsive phosphoproteome of the proximal tubule, and how changes in the expression and phosphorylation of RNA-binding proteins may affect Npt2a mRNA stability. 
In Aim 1, we found that PTH decreases Npt2a steady-state mRNA levels with a $50 \%$ loss in $2.2 \mathrm{~h}$ compared to $8.6 \mathrm{~h}$ in the absence of PTH. This effect is posttranscriptional, and is dependent on both transcription and translation. In Aim 2, we determined that PTH destabilizes Npt2a mRNA through both PKA- and PKC-dependent mechanisms. The rapid initial decline in Npt2a mRNA levels corresponds to and appears to be dependent on early PTH-stimulated PKA activation. In contrast, PTH-stimulated PKC activation occurs more gradually over several hours and likely contributes to the latter phase of Npt2a mRNA destabilization. Finally, in Aim 3, we found that PTH alters the phosphorylation status of almost 1200 proteins in the proximal tubule, including 68 RNA-binding proteins (RBPs). Two of those RBPs, KHSRP and Roquin-2, bind to Npt2a mRNA, and their expression varies inversely with Npt2a mRNA levels. From these studies, we conclude that PTH-mediated Npt2a mRNA destabilization likely occurs through PKA- and PKC-dependent modulation of RBP expression and activity, and that KHSRP and Roquin-2 are potential mediators of the PTH response. 
TABLE OF CONTENTS

PAGE

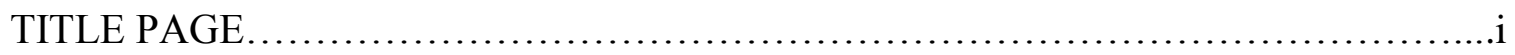

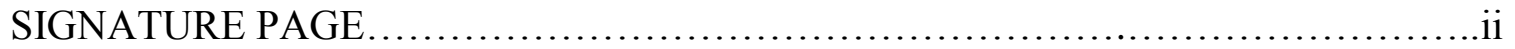

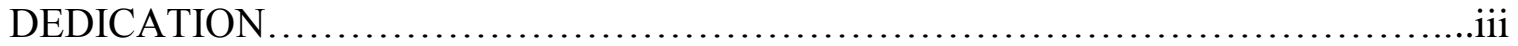

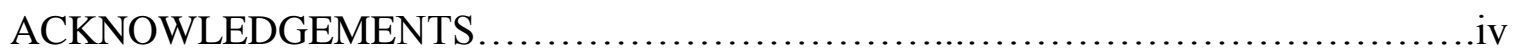

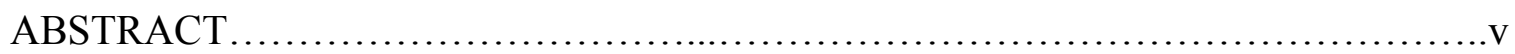

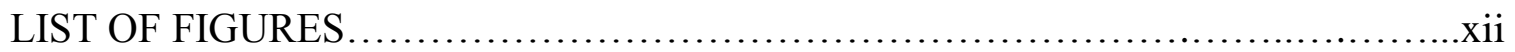

CHAPTER I: INTRODUCTION

I. "Where there's life there's phosphorus"...................................

Pathophysiology of phosphorus...........................................

Determinants of serum phosphorus........................................ 4

Contribution of phosphate transporters to renal phosphate reabsorption........6

Regulation of serum phosphorus...................................... 8

Effects of PTH on proximal tubule ion transport and metabolism............12

\section{CHAPTER II: PRELIMINARY DATA}

I. Npt2a expression in the proximal tubule...............................15

Discrepant effects of acute versus chronic PTH..........................16

\section{CHAPTER III: RESEARCH PROPOSAL}

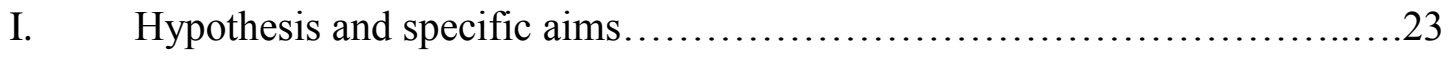

\section{CHAPTER IV: SPECIFIC AIM I}

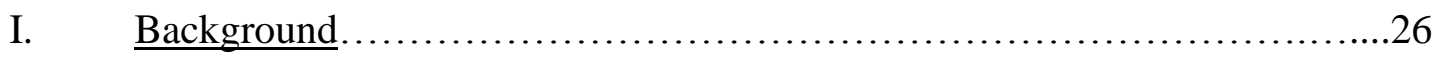

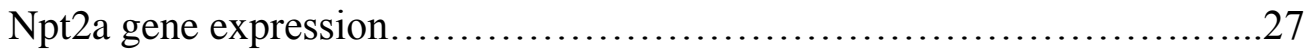


Post-transcriptional regulation of Npt2a.

Evidence for a role of PTH in the regulation of $\mathrm{Npt} 2 \mathrm{a}$ gene

expression................................................. 30

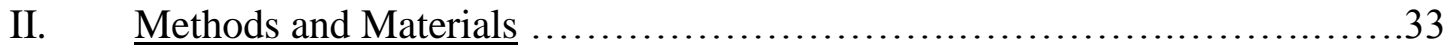

Materials.......................................................... 33

PTH-D1 mouse model.............................................33

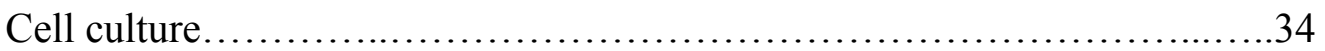

Immunohistochemistry ..........................................

Brush-border membrane and crude membrane isolation....................36

Protein determination................................................ 36

Western blot analysis............................................. 37

Isolation of total RNA ............................................... 37

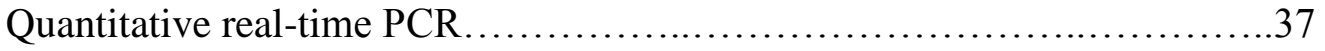

Luciferase reporter gene assays..................................... 38

Statistics...................................................... 39

Ethical aspects of the proposed research.............................39

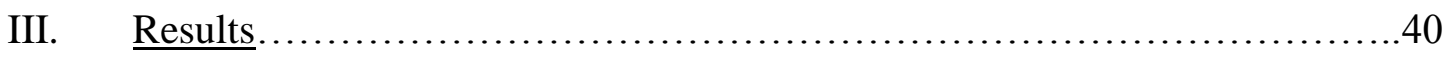

Part 1: Effect of chronic primary hyperparathyroidism on renal tubular proteins.....................................40

Part 2: Time-dependent effects of PTH on Npt2a mRNA..................43

Part 3: Roles of transcription and translation in Npt2a mRNA stability......44

Part 4: Effect of PTH on Npt2a promoter activity......................47

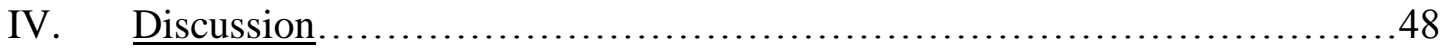


V. Conclusion.

\section{CHAPTER V: SPECIFIC AIM II}

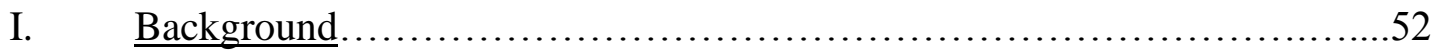

The PTH receptor signaling complex.................................52

PTH-induced PKA signaling ...........................................55

PTH-induced PKC signaling...........................................56

PTHR1 endocytosis and desensitization................................58

Pathways involved in Npt2a protein downregulation......................59

Modification of receptor coupling and signaling through PTH fragments.....62

PTH signaling and Npt2a mRNA regulation.............................63

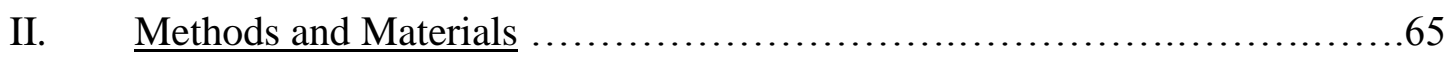

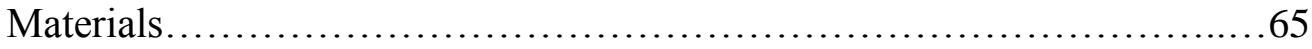

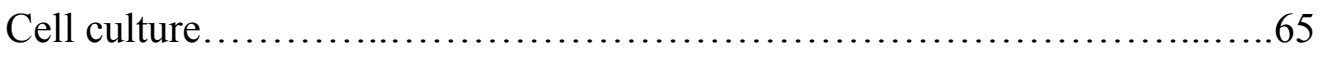

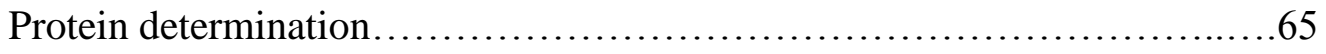

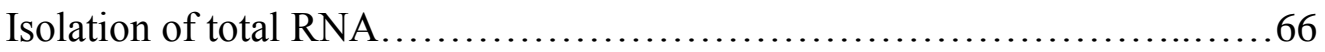

Quantitative real-time PCR ...........................................66

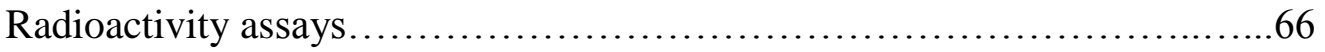

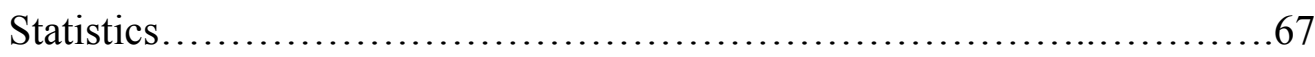

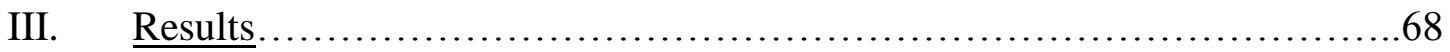

Part 1: Effects of PTH analogues on Npt2a mRNA expression ..............68

Part 2: Effects of direct activation of PKA vs PKC on Npt2a mRNA

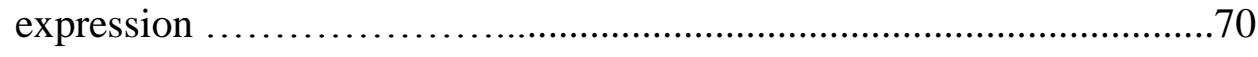

Part 3: Effects of select pathway inhibition on PTH-mediated 
Npt2a mRNA destabilization

Part 4: Effect of inhibition of endocytosis on PTH-mediated

Npt2a mRNA destabilization ....................................... 75

IV. Discussion......................................................... 76

V. Conclusion...................................................... 83

\section{CHAPTER VI: SPECIFIC AIM III}

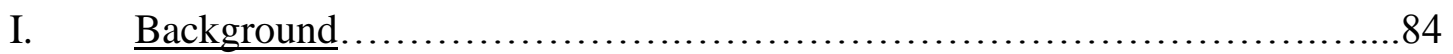

Regulation of mRNA expression....................................85

Protein-induced mRNA decay.......................................87

The 3' UTR of Npt2a mRNA.....................................87

The PTH-responsive phosphoproteome...............................88

II. Methods and Materials ............................................... 91

Materials........................................................91

PTH-D1 mouse model..........................................91

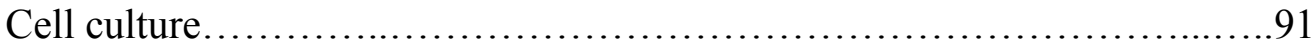

Protein determination..............................................91

Liquid chromatography-mass spectrometry ........................92

Gene identification and pathway analysis..........................99

Computational analysis of RNA-protein interaction prediction.............93

Isolation of crude membrane, crude nucleus, and cytosol..................93

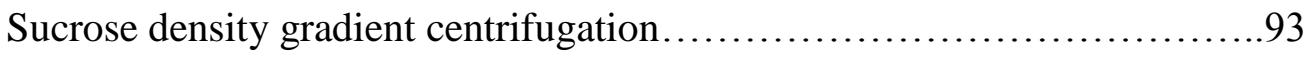

Western blot analysis.......................................... 94

RNA-binding protein immunoprecipitation (RIP) ........................94 
Isolation of RNA .................................................95

Reverse transcription and real-time PCR.............................95

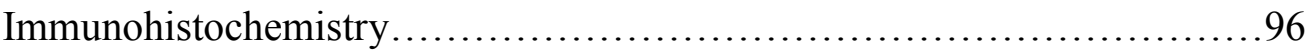

Statistics..........................................................96

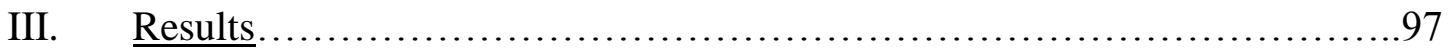

Part 1: The PTH-responsive phosphoproteome .......................97

Part 2: Regulation of KHSRP and Roquin-2 Expression by PTH...........105

Part 3: Interaction of KHSRP and Roquin-2 with Npt2a mRNA...........107

IV. Discussion........................................................ 111

V. Conclusion......................................................... 117

CHAPTER VII: DISCUSSION AND CONCLUSIONS $\ldots \ldots \ldots \ldots \ldots \ldots \ldots \ldots \ldots \ldots \ldots$

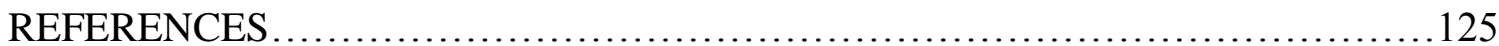

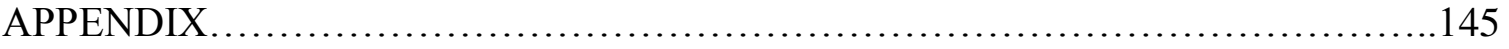

CURRICULUM VITA ......................................................... 148 


\section{LIST OF FIGURES}

FIGURE

PAGE

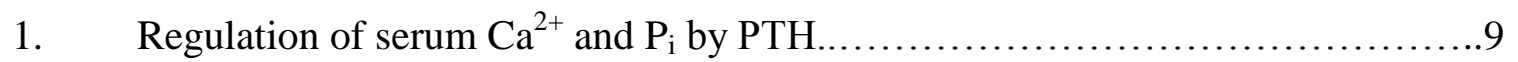

2. Effects of acute PTH on the proximal tubule ..................................13

3. Effect of acute and chronic PTH on proximal tubule proteins...................18

4. Effect of PTH on BBM and BLM protein expression in proximal tubule cells...19

5. Effect of PTH on Npt2a and NHE3 mRNA expression........................20

6. Proximal tubule protein expression in response to chronic PTH. ................41

7. Npt2a mRNA expression in PTH-D1 mice.................................42

8. Npt2a mRNA expression in response to PTH in OK cells.....................43

9. Role of transcription in Npt2a mRNA regulation.............................45

10. Role of translation in Npt2a mRNA regulation.............................46

11. Effect of PTH on Npt2a promoter function.................................47

12. PTH-receptor binding and intracellular signaling in the proximal tubule.........57

13. Effect of pathway-specific PTH analogues on Npt2a mRNA......................69

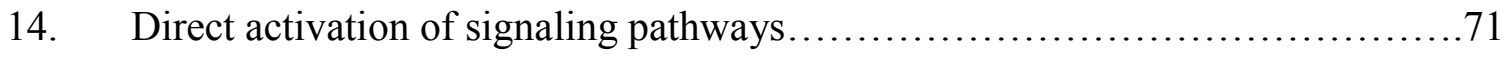

15. Effect of PTH on Npt2a mRNA during selective pathway inhibition.............73

16. Effect of endocytosis inhibition on PTH-mediated Npt2a mRNA

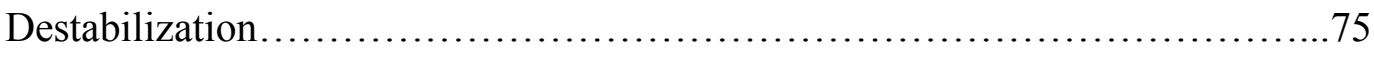

17. Summary of effects of selective PKA or PKC activation on Npt2a mRNA......77 
18: $\quad$ Predicted secondary structure of the 3' UTR of Npt2a mRNA..................89

19: $\quad$ PTH-responsive phosphoproteome........................................ 98

20: $\quad$ KHSRP expression in the proximal tubule.............................. 106

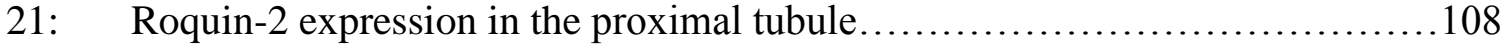

22: Interaction of KHSRP and Roquin-2 with Npt2a mRNA..................110

23: $\quad$ Proposed model of PTH-mediated Npt2a mRNA destabilization...............120 


\section{CHAPTER I}

\section{INTRODUCTION}

"Where there's life there's phosphorus"

Since the initial isolation of adenosine triphosphate from muscle extracts in 1929, phosphate has been at the heart of physiology. The aforementioned quote is known as Todd's Hypothesis, after the biochemist Alexander Todd (1907-1997) who was the first to chemically synthesize the ATP molecule in 1948, for which he later won the Nobel Prize in $1958^{1}$. The phosphate ester bond is a uniquely flexible chemical bond, with the ability to form extremely stable bonds capable of enduring millions of year (DNA) as well as extremely transient bonds that last mere milliseconds. In the years following the discovery of ATP, scientists have uncovered numerous biological processes in which phosphate is a critical element. At the underpinning of life as we know it, phosphate is essential in formation of deoxy- and ribonucleic acids ${ }^{2}$. Apart from being an integral part of the genetic code, the second major function of phosphate within the body is to provide whole organism structure through the development and maintenance of the skeleton. In the form of ATP, phosphate is also an essential component of the ubiquitous energy currency for cellular metabolism ${ }^{3-5}$. Intracellular signaling and secondary messenger cascades are heavily reliant on the use of phosphate as an on- or off-switch in the regulation of enzymatic activity and protein function ${ }^{6}$. 
Within the body, phosphate is a ubiquitous mineral, and is present as inorganic phosphate, the vast majority of which is located within bone and teeth (85\%), with the bulk of the remainder being found in soft tissues (14.3\%); interstitial fluid, plasma, and red blood cells each account for less than three-tenths of a percent of the total body phosphate content ${ }^{7}$. In addition to the major physiological functions previously mentioned, phosphate also plays several other roles within the body. Divalent phosphate $\left(\mathrm{HPO}_{4}{ }^{2-}\right)$ is an important physicochemical buffer, maintaining $\mathrm{pH}$ balance during states of acidosis. It provides structure not only at the macromolecular level in the form of bone mineralization and formation, but also at the cellular level, providing organization and compartmentalization through the stability and amphipathic nature of phospholipids.

Phosphate also aids in oxygen transport through the interaction of 2,3-diphosphoglycerate with hemoglobin ${ }^{7}$. As a crucial biological mineral, phosphate and the processes underlying its homeostasis remain significant areas of research.

\section{Pathophysiology of phosphorus}

The fact that total body phosphate homeostasis is crucial for cardiovascular health is one of the most significant findings of the last two decades ${ }^{8-10}$. Dysregulation of phosphate homeostasis, including both hyperphosphatemia and hypophosphatemia, can result in severe pathophysiological conditions. Normal clinical values for serum phosphorus have been defined as 3.4 to $4.5 \mathrm{mg} / \mathrm{dL}^{11}$. Even mild hyperphosphatemia with serum values of $5.5 \mathrm{mg} / \mathrm{dL}$ or greater correlate with a statistically significant increase in the risk of death from cardiovascular and renal diseases ${ }^{12}$. Increased serum phosphorus accompanying chronic kidney disease is associated with such cardiovascular complications as vascular calcification and toxicity ${ }^{13,14}$. In addition to elevated serum 
phosphorus leading to increased precipitation of calcium-phosphate deposits within the body, research indicates that the presence of excess phosphate itself can be detrimental to cell function, particularly in cardiovascular cells. Vascular smooth muscle cells cultured in a high phosphorus medium displayed upregulated expression of bone-promoting differentiation markers that facilitate extracellular matrix calcification ${ }^{15,16}$. Phosphate has been implicated as a pro-aging factor ${ }^{6}$, producing not only vascular pathologies such as ectopic calcification and arteriosclerosis, but also contributing to the development of COPD, chronic kidney disease, and hearing loss. Aside from cardiovascular complications, it is thought that diets chronically high in phosphorus strongly contribute to bone disorders in aging ${ }^{17}$. Accumulation of phosphate has even been implicated in the development of muscle fatigue during prolonged exercise ${ }^{18}$.

Just as high serum phosphorus produces increased cardiovascular morbidity and mortality, too little phosphorus can produce pathologies just as detrimental. Hypophosphatemia can have severe cardiovascular repercussions including vasodilation, hemolysis, and cardiac failure ${ }^{19}$. Additionally, many bone diseases are attributed to hypophosphatemia. Perhaps most notably, the hereditary skeletal disorder autosomal dominant hypophosphatemic rickets results from renal phosphate wasting, producing severe bone malformation ${ }^{20}$. Other bone diseases also resulting from hypophosphatemia include X-linked hypophosphatemia as well as hereditary hypophosphatemic rickets with hypercalciuria. Phosphate is a crucial component of the bone mineral hydroxyapatite, and hypophosphatemia not only causes decreased bone mineralization and fragility but also pain and growth retardation ${ }^{21}$. With so many roles in the body and so many potential pathologies from the dysregulation of phosphate, its maintenance and regulation thus 
represent a crucial component of cardiovascular and bone health, with serious implications for clinical morbidity and mortality.

\section{Determinants of serum phosphorus}

Total body phosphate homeostasis is tightly maintained through a complex axis involving the intestines, kidneys, and bones. While the minimum daily dietary requirements for phosphorus are approximately $20 \mathrm{mmol} / \mathrm{day}^{7}$, the Western diet typically contains a much higher amount than stipulated by metabolic needs. In adults, serum phosphorus levels result from a combination of dietary intake, bone modeling, and kidney excretion rates, the last of which is the most crucial determinant of serum phosphorus. Dietary phosphate can be obtained from all natural foods, with particular abundance in protein-rich food as well as cereal grains ${ }^{2}$. Phosphorus obtained from animal sources is more bioavailable and more readily absorbed than phosphate obtained from plant sources $^{22}$. In more recent years, dietary loads of phosphate have increased through the heavy reliance of the food industry on phosphate-rich food additives to preserve food shelf life $\mathrm{e}^{22}$. In the steady state, however, the amount of phosphate absorbed from the diet is balanced by the amount of phosphate excreted by the kidney.

In the gut, absorption of dietary phosphate into the circulation occurs through paracellular pathways and through transcellular pathways mediated primarily by the type IIb sodium-phosphate cotransporter (Npt2b), expressed in the apical membrane of the duodenum and early jejunum ${ }^{17}$. While Npt2b expression is known to be regulated by intestinal phosphate content, the specific mechanisms governing regulation of intestinal phosphate transport remains poorly understood. In addition to the contribution of the gut to phosphate homeostasis, bone also plays a major role in phosphate homeostasis, as the 
hydroxyapatite matrix serves as a critical pool of phosphorus and calcium reserves. Adult bones undergo constitutive remodeling, with phosphate and calcium cycling into and out of the bones and circulation as osteoclastic bone resorption is countered by osteoblastic bone formation ${ }^{23}$. The flux of phosphate into and out of bone is normally balanced in the adult, and thus serum phosphorus levels are mostly determined by the rate of renal excretion.

In the kidney, approximately one-fifth of renal plasma flow is filtered into Bowman's space. This renal ultrafiltrate then enters the nephron, wherein water and solutes will either be reclaimed back into the circulation, or pass through to be excreted in the final urine. A small solute, phosphate is freely filtered at the glomerulus, and its reabsorption within the nephron occurs almost exclusively in the proximal tubule ${ }^{24}$. Phosphate does not undergo secretion within any segment of the nephron, and thus rates of renal phosphate excretion are dependent on the rate of phosphate reabsorption from the ultrafiltrate of the glomerulus. The proximal tubular transport maximum for phosphate reabsorption is directly related to the abundance of phosphate transporters expressed within the apical membrane. Once the transport maximum is reached, any excess filtered phosphate will end up in the final urine. Phosphate is reclaimed from the ultrafiltrate through secondary-active transport coupled to the movement of sodium down its concentration gradient, which allows for entry of phosphate from the tubule lumen into the tubular epithelial cell. Reabsorption of phosphate back into the bloodstream is achieved by phosphate exiting the cell across the basolateral membrane through unknown mechanisms. 


\section{Contribution of phosphate transporters to renal phosphate reabsorption}

Mammalian phosphate transport is accomplished by two families of secondaryactive sodium-coupled phosphate transporters: SLC20 and SLC34. The SLC20 family is comprised of PiT-1 (SLC20A1) and PiT-2 (SLC20A2). PiT-1 and PiT-2 mRNAs are ubiquitously expressed in both murine and human tissue, and they are thought to serve a housekeeping function of providing phosphate for intracellular metabolic needs ${ }^{25,26}$. Within the kidney, although expression of both PiT-1 and PiT-2 has been found, only PiT-2 has been identified in the apical membrane of the proximal tubule ${ }^{27}$.

The SLC34 family is comprised of Npt2a (SLC34A1) and Npt2c (SLC34A3). Both Npt2a and Npt2c are expressed in the apical membrane of the proximal tubule, with Npt2a expression seen throughout the proximal tubule, while Npt2c expression exhibits heavier expression in the early segments of juxtaglomerular proximal tubules. Npt2a is an electrogenic transporter, cotransporting three sodium ions and one divalent phosphate ion into the cell $\left(3 \mathrm{Na}^{+}: 1 \mathrm{HPO}_{4}{ }^{2-}\right)$, whereas $\mathrm{Npt} 2 \mathrm{c}$ is electroneutral, coupling the entry of two sodium ions and one divalent phosphate ion $\left(2 \mathrm{Na}^{+}: 1 \mathrm{HPO}_{4}{ }^{2-}\right)$. Both $\mathrm{Npt} 2 \mathrm{a}$ and $\mathrm{Npt} 2 \mathrm{c}$ are the primary mediators of proximal tubule reclamation of filtered phosphate with PiT-2 contributing only a small fraction to overall phosphate reabsorption ${ }^{20,28,29}$.

In rodents, the type IIa sodium-phosphate cotransporter (Npt2a) has been identified as the transporter responsible for the majority of phosphate reabsorption, accounting for up to $80 \%$ of brush-border phosphate transport ${ }^{30-32}$. Studies in mice support a preeminent role for $\mathrm{Npt} 2 \mathrm{a}$ in contributing to overall phosphate homeostasis, as Npt2a knockout mice not only display significant phosphate wasting, but they also develop hypercalciuria with tubular and interstitial calcium-phosphate deposits, and 
display developmental skeletal abnormalities ${ }^{33-35}$. Ablation of the Npt2c gene in mice does not elicit a hypophosphatemic phenotype, with no apparent disturbances in calcium or phosphate homeostasis ${ }^{36}$. Studies of protein dynamics in the proximal tubule show that in response to $\mathrm{PTH}, \mathrm{Npt} 2 \mathrm{a}$ is endocytosed and sent to the lysosomes for degradation, whereas Npt2c is endocytosed but escapes lysosomal degradation, which suggests that control of Npt2a expression may be more important for rapid and sustained changes in proximal tubule phosphate reabsorption ${ }^{37}$.

The contribution of the renal phosphate transporters to overall phosphate homeostasis may be species-specific, however. Clinical syndromes associated with Npt2a and $\mathrm{Npt} 2 \mathrm{c}$ gene mutations suggest that $\mathrm{Npt} 2 \mathrm{c}$ expression and function may be more important for total phosphate homeostasis than Npt2a in humans. Loss-of-function mutations in the human $\mathrm{Npt} 2 \mathrm{c}$ gene have been identified as the cause of hereditary hypophosphatemic rickets with hypercalciuria and accompanying nephrolithiasis renal phosphate wasting $^{6,38}$. In contrast, human Npt2a mutations were found to be associated with a considerably milder phenotype of osteoporosis and nephrolithiasis ${ }^{39,40}$. However, recent clinical reports suggest a more significant role for $\mathrm{Npt} 2 \mathrm{a}$ expression and function in human phosphate homeostasis than previously thought. Magen et al. ${ }^{41}$ reported a clinical case of autosomal recessive hypophosphatemic rickets with renal Fanconi's Syndrome secondary to an Npt2a loss-of-function genetic mutation. Likewise, Rajagopal et al. ${ }^{42}$ and Kenny et al. ${ }^{43}$ both reported case studies of mutations in the Npt2a gene that resulted in phenotypes of renal phosphate wasting with resultant hypercalcemia, hypercalciuria, and hypophosphatemia. While these clinical reports suggest that the contribution of specific renal phosphate transporters to overall phosphate homeostasis 
may be more nuanced than previously recognized, they provide evidence and support for Npt2a as an important determinant of serum phosphorus, as well as a critical target of regulators of renal phosphate reabsorption.

\section{$\underline{\text { Regulation of serum phosphorus }}$}

The maintenance of serum phosphate is dependent on the rate of its reabsorption in the kidney, which is subject to strict regulation by a host of factors, including diet and hormones. Npt2a expression and function are determined by physiologic regulators of serum phosphorus, including parathyroid hormone (PTH), one of the major phosphaturic hormones ${ }^{34}$. The amount of phosphate in the diet regulates phosphate reabsorption in the kidney, independent of changes in serum phosphorus, as low-phosphate diets upregulate proximal tubular phosphate reabsorption prior to any changes in serum phosphorus ${ }^{44}$. Apart from dietary regulation, phosphate reabsorption is also modulated by a host of hormonal factors. Several factors that increase renal phosphate reabsorption include growth hormone, insulin-like growth factor 1, insulin, thyroid hormone, and stanniocalcin. These factors, however, play a minor role in phosphate homeostasis as compared to the major hormonal regulators of renal phosphate reabsorption - PTH, fibroblast growth factor 23 (FGF23), and vitamin $\mathrm{D}^{6,45}$.

PTH is an 84-amino acid peptide hormone that is released from the parathyroid glands in response to either decreased serum $\mathrm{Ca}^{2+}$ or increased serum $\mathrm{P}_{\mathrm{i}}^{46}$. In bone, PTH stimulates osteoclastic activity indirectly through its effect on osteoblast secretion of paracrine factors $^{2,47}$. As shown in Figure 1, bone resorption as a result of PTH stimulation releases $\mathrm{Ca}^{2+}$ and $\mathrm{P}_{\mathrm{i}}$ into the circulation. In the proximal tubule, PTH upregulates $1 \alpha-$ 
hydroxylase expression, which enhances production of 1,25-dihydroxy-vitamin D,

promoting increased calcium absorption in the gut ${ }^{48}$. In the process of releasing calcium from bone, phosphate stores also become liberated. To excrete the excess phosphate and preserve the newly restored serum calcium level, PTH exerts a phosphaturic effect in the

\section{FIGURE 1}

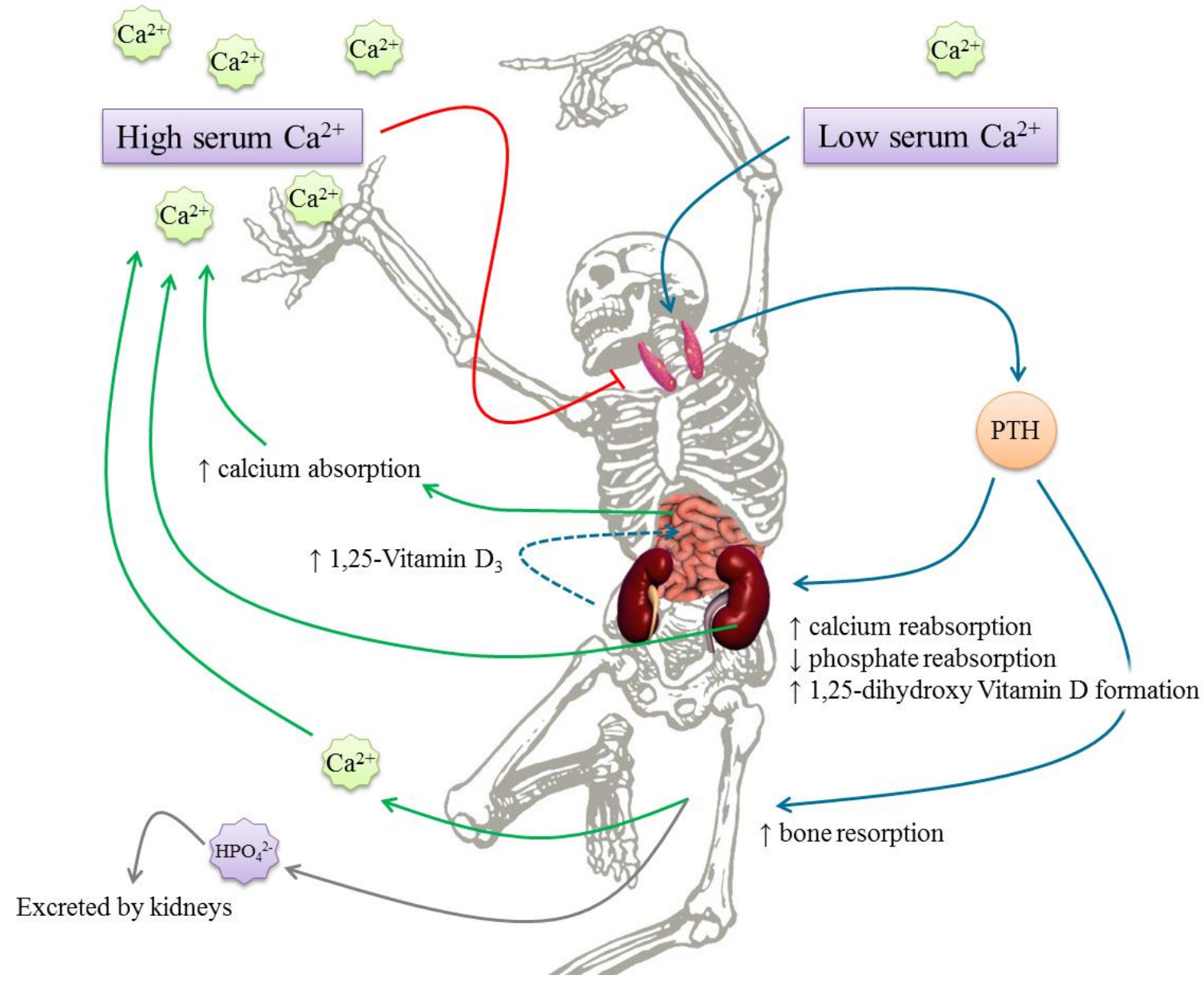

Figure 1: Regulation of serum $\mathrm{Ca}^{2+}$ and $\mathbf{P}_{\mathbf{i}}$ by PTH. Low serum $\mathrm{Ca}^{2+}$ stimulates the release of PTH from the parathyroid gland. PTH acts on the bone to increase bone resorption, releasing $\mathrm{Ca}^{2+}$ and $\mathrm{HPO}_{4}{ }^{2-}$ into the bloodstream. At the kidney, PTH increases $\mathrm{Ca}^{2+}$ reabsorption and decreases $\mathrm{HPO}_{4}{ }^{2-}$ reabsorption, maintaining the elevated serum $\mathrm{Ca}^{2+}$ from the resorption of bone. Vitamin $\mathrm{D}$ becomes activated in the kidney by $1 \alpha-$ hydroxylase, leading to increased $\mathrm{Ca}^{2+}$ absorption from the gut. The restored serum $\mathrm{Ca}^{2+}$ provides a negative feedback signal to the parathyroid glands, discontinuing the release of PTH. 
kidney to decrease the reabsorption of phosphate from the plasma ultrafiltrate, while simultaneously increasing calcium reabsorption in the distal tubule to sustain the elevation in serum calcium ${ }^{7,49}$. As serum PTH levels increase, the renal transport maximum for phosphate decreases ${ }^{50}$, which is accomplished through the downregulation of both of the major renal phosphate transporters, Npt2a and Npt $2 c^{51}$.

FGF23 is secreted from the bone and is another potent phosphaturic hormone that in conjunction with Klotho decreases the renal transport maximum for phosphate by decreasing the abundance of Npt2a and Npt2c, as well as PiT-2, in the BBM of the proximal tubule ${ }^{52}$. Klotho is the co-receptor for FGF23, and also circulates as an endocrine factor to promote anti-aging systemic effects ${ }^{54}$. FGF23 exerts its hypophosphatemic effects independent of and synergistically with $\mathrm{PTH}^{53}$, and plays a prominent role throughout the development of chronic kidney disease (CKD), as FGF23 expression increases exponentially with the progressive decline of kidney function and resultant increase in serum phosphate ${ }^{54}$. Consistent with these effects, FGF23overexpressing mice display hypophosphatemia with decreased transcript and protein expression of $\mathrm{Npt}_{2} \mathrm{a}^{55}$. Conversely, mice with FGF23 gene ablation display increased serum phosphate, increased apical expression of $\mathrm{Npt} 2 \mathrm{a}$, and an accompanying increase in the renal phosphate transport maximum ${ }^{53}$. Distal-tubule-specific deletion of Klotho also produces a phenotype of hyperphosphatemia with abundant Npt2a BBM expression ${ }^{56}$. However, the actions of FGF23 on Npt2a expression are distinct from the actions of PTH, as PTH-induced phosphaturia is elicited through PKA- and PKC-dependent pathways, whereas FGF23-mediated phosphaturia occurs via MAP kinase activation ${ }^{57,58}$. 
Additionally, PTH appears to be a calcium-centric hormone, with secondary effects on phosphate homeostasis, whereas FGF23 is more phosphate-centric.

While the actions of PTH and FGF23 on proximal tubular phosphate reabsorption are direct and relatively straight-forward, the actions of vitamin D on phosphate reabsorption, and Npt2a in particular, are complex. Acutely, vitamin D appears to stimulate phosphate reabsorption by increasing expression of Npt2a through upregulation of Npt2a promoter activity in a cell-specific manner, although this action appears to have a weak direct influence on phosphate transport ${ }^{59}$. Rather, vitamin D appears to play a more important role in overall phosphate homeostasis by modulating the FGF23/Klotho/PTH bone-endocrine axis ${ }^{6}$. PTH stimulates the formation of active vitamin D, which, in a negative feedback loop, suppresses further PTH release from the parathyroid glands ${ }^{60,61}$. Vitamin D also increases FGF23 expression, which decreases renal $1 \alpha$-hydroxylase expression in addition to promoting phosphaturia ${ }^{62}$. Despite vitamin D's direct and indirect actions in the proximal tubule to increase apical membrane phosphate transport, chronic exposure to vitamin D has actually been shown to decrease Npt2a expression synergistically with PTH, likely in response to increased phosphate release from increased bone turnover ${ }^{63}$.

PTH and FGF23 are the most prominent regulators of serum phosphorus, although other important physiological factors have also been shown to play a more minor role in phosphate homeostasis. Secreted frizzled-related protein 4 (Sfrp4) has been implicated as an acute downregulator of Npt2a expression in the proximal tubule, but genetic ablation studies were unable to show a chronic physiologically-relevant role of this protein in overall phosphate homeostasis ${ }^{64}$. Estrogen has phosphaturic actions that 
induces phosphate wasting through the downregulation of Npt2a mRNA and protein expression, but produces no effect on the expression of either Npt2c or PiT $-2^{5}$. Other regulators of serum phosphate with less dramatic effects on renal phosphate transport include dopamine, PTH-related-peptide, calcitonin, atrial natriuretic peptide, epidermal growth factor, and glucocorticoids ${ }^{53,65}$. While these and other factors regulate renal phosphate transport, PTH remains a critical determinant of phosphate reabsorption within the proximal tubule, and serves as an important hormonal regulator of proximal tubule function.

\section{Effects of PTH on proximal tubule ion transport and metabolism}

The effects of PTH on the proximal tubule are not limited to phosphate transport alone. As shown in Figure 2, PTH regulates several ion transporters and metabolic pathways. PTH stimulates gluconeogenesis through the actions of adenylyl cyclase ${ }^{66,67}$, as well as ammoniagenesis through the increased catabolism of glutamate ${ }^{68}$. In the kidney, reabsorption of filtered bicarbonate is dependent on sodium-hydrogen exchange across the apical membrane of the proximal tubule, a process that is also decreased by PTH through the decreased expression and activity of the sodium/hydrogen exchanger isoform $3(\mathrm{NHE} 3)^{69,70}$. Coupled with the downregulation of the sodium/potassiumATPase $\left(\mathrm{Na}^{+} / \mathrm{K}^{+}\right)$, PTH decreases the transcellular transport of sodium within the proximal tubule, an effect that over time would tend to produce volume depletion and hypotension $^{49,71}$. 
Due to its systemic effects on the kidney, bone, and gut, PTH plays a crucial role in the maintenance of total body calcium and phosphate homeostasis. Imbalances in phosphate and calcium occur in the case of primary hyperparathyroidism, wherein

\section{FIGURE 2}

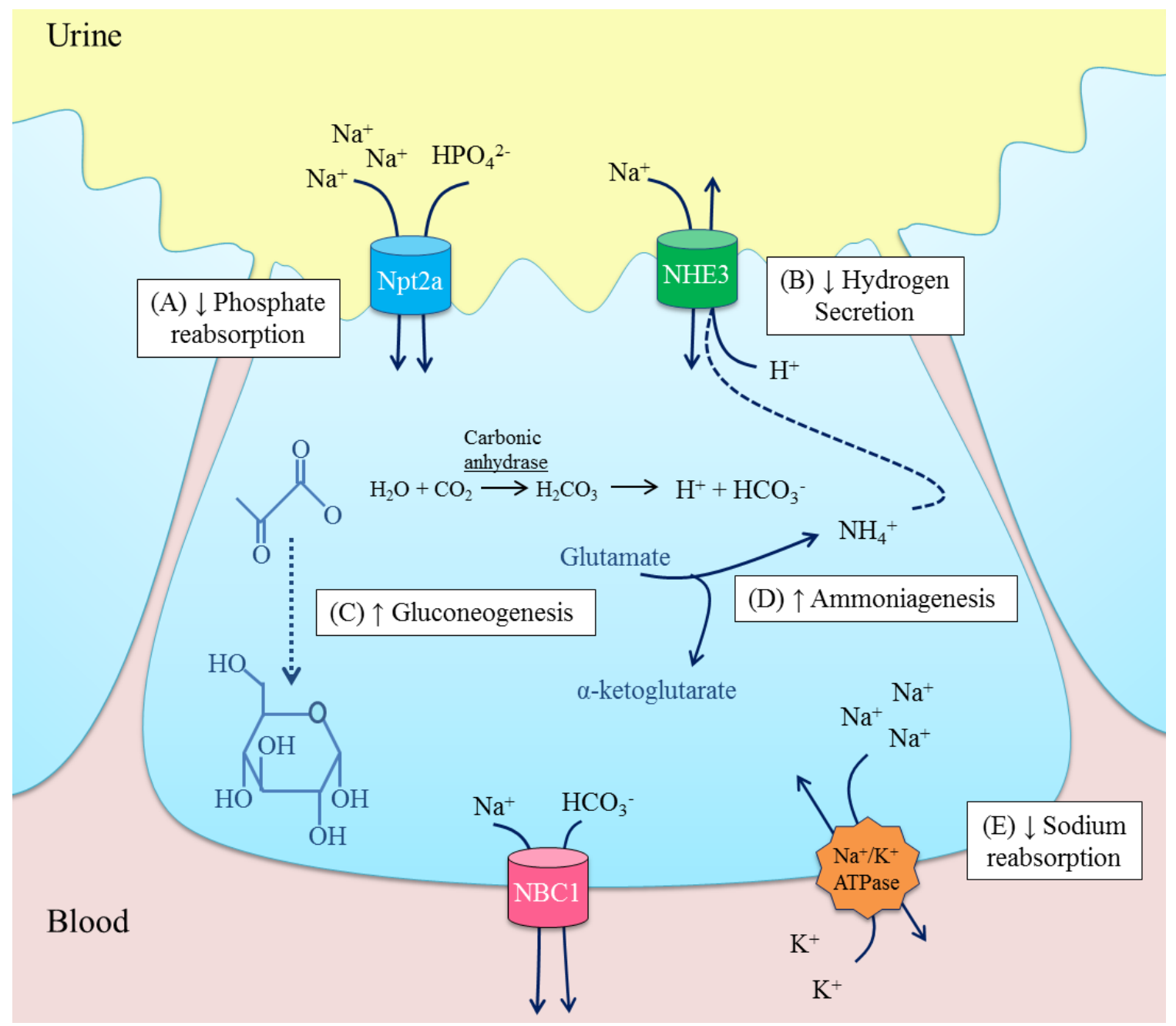

Figure 2: Effects of acute PTH on the proximal tubule. Acute stimulation of the proximal tubule by PTH results in (A) decreased hydrogen secretion through downregulation of NHE3, (B) decreased phosphate reabsorption through down-regulation of NpT2a, (C) increased gluconeogenesis, (D) increased ammoniagenesis, and (E) decreased sodium reabsorption through down-regulation of $\mathrm{Na}^{+} / \mathrm{K}^{+}$-ATPase. 
chronic elevated serum PTH causes increased serum calcium and decreased serum phosphorus levels. Accordingly, patients with untreated primary hyperparathyroidism display higher incidences of mineral disorders, including aortic valve calcification, hypertension, and kidney stones ${ }^{72,73}$. Acute stimulation of the proximal tubule by PTH results in downregulation of bicarbonate, sodium, and phosphate reabsorption, but clinical observations suggest that the chronic effects of PTH differ from the acute effects. Patients with primary hyperparathyroidism do not consistently display volume depletion or metabolic acidosis ${ }^{74,75}$, which would be expected from sustained downregulation of NHE3 and $\mathrm{Na}^{+} / \mathrm{K}^{+}$. Whereas many PTH responses in the proximal tubule seemingly desensitize over time, downregulation of phosphate reabsorption persists. The studies presented within this dissertation seek to clarify the mechanism for the chronic downregulation of Npt2a by PTH. 


\section{CHAPTER II}

\section{PRELIMINARY DATA}

\section{Npt2a expression in the proximal tubule}

The rate of phosphate reabsorption in the proximal tubule is directly related to the relative abundance and stability of Npt2a in the apical membrane. The expression of $\mathrm{Npt} 2 \mathrm{a}$ in the brush border membrane is heavily reliant on its interaction with the scaffolding protein NHERF-1 (sodium-hydrogen exchanger regulatory factor 1). As its name implies, NHERF-1 was originally identified for its role in the regulation of NHE3, but has since been identified as a protein with more global function than just regulation of sodium-hydrogen exchange $\mathrm{e}^{45,76}$. Of the population of Npt2a present in the apical membrane of the proximal tubule, approximately $35-50 \%$ is bound to NHERF-1, and this interaction stabilizes $\mathrm{Npt} 2 \mathrm{a}$ in the membrane and prolongs its retention there ${ }^{77}$. NHERF-1 contains two PDZ domains - a conserved motif among scaffolding proteins that is crucial for its interaction with other proteins. Mutation analyses have shown the PDZ-1 domain of NHERF-1 to be critical not only for NHERF1-Npt2a associations, but also for regulated membrane expression ${ }^{50}$. As an indication of its biological importance, the NHERF-1 PDZ domain is highly conserved across species ${ }^{50}$, and serves as a regulatory target for several hormones, including PTH. NHERF-1 assembles a complex of proteins involved in PTH receptor (PTHR1) signaling, including PTHR1, PLC $\beta$, AKAP79/150, 
and $\operatorname{actin}^{78}$, and stabilizes the apical expression of those complexes through its interaction with the cytoskeleton ${ }^{79}$. Accordingly, NHERF-1 is also required for maximal PTHresponsive inhibition of phosphate uptake ${ }^{80}$. In addition to the assembly of the PTH receptor complex, however, NHERF-1 also augments phosphate uptake inhibition by 8Br-cAMP, PMA, and forskolin (activating PKA, PKC, and adenylyl cyclase, respectively), indicating a role for NHERF-1 in PTH receptor signaling beyond the assembly of the PTHR1 complex ${ }^{80}$.

PTH-stimulated endocytosis of Npt2a occurs through the phosphorylation of a serine residue in the PDZ domain of NHERF- $1^{77}$, which induces the rapid dissociation of Npt2a from NHERF-1. Maximal disassociation occurs 30 to 40 minutes following PTH stimulation $^{32}$. Whereas Npt2a undergoes rapid endocytosis in response to PTH, NHERF1 remains present in the membrane, as an important regulator for other ion transporters and protein complexes not involved in phosphate transport ${ }^{32}$. Following endocytosis to the endosomal compartment, Npt $2 \mathrm{a}$ is targeted to lysosomes for degradation ${ }^{81,82}$. Reinsertion of Npt2a back into the apical membrane following removal of the PTH stimulus requires de novo synthesis, and thus takes several hours before new Npt2a begins to repopulate the apical membrane ${ }^{83}$.

\section{Discrepant effects of acute versus chronic PTH}

We examined through immunohistochemistry the expression of Npt2a and the $\alpha$ subunit of $\mathrm{Na}^{+} / \mathrm{K}^{+}$in acutely versus chronically PTH-treated Sprague-Dawley rats. To mimic the conditions of hyperparathyroidism, the rats were administered exogenous PTH ( $5 \mu \mathrm{g}$ PTH/kg body weight) for either 6 hours (acute exposure) or 4 days (chronic 
exposure, injected once daily). In comparison to saline-treated rats, both acutely and chronically treated rats displayed significantly decreased expression of Npt2a (Figure 3A, next page), as opposed to the alpha subunit of the sodium pump, which showed no significant change in expression between control and PTH-treated kidneys (Figure 3B). In addition to studying PTH-regulated proteins in the Sprague Dawley rat kidney, we also explored the chronic regulation of membrane proteins in a cell culture model of the proximal tubule, the opossum kidney (OK) cell, as described in Chapter IV Methods. OK cells were treated with either $2 \mathrm{~h}$ PTH to mimic acute PTH, or 3d PTH (media replaced daily) to mimic chronic PTH stimulation. Brush-border membranes (BBM) and basolateral membranes (BLM) were isolated following PTH treatment, and protein expression was quantified by Western blot. As shown in Figure 4A (page 19), treatment of cells with PTH produced a rapid and significant reduction in Npt2a BBM expression, and this effect was sustained with chronic PTH. Expression of NHE3 did not change with acute PTH, and actually displayed a significant increase with chronic PTH. Neither NHERF-1 (Figure 4A) nor PTH receptor (PTHR1, Figure 4B) BBM expression changed with either acute or chronic PTH stimulation. Isolation of basolateral membranes from PTH-treated cells showed that expression of the $\alpha$-subunit of the $\mathrm{Na}^{+} / \mathrm{K}^{+}$-ATPase also did not significantly change with PTH treatment (Figure 4C). 


\section{FIGURE 3}

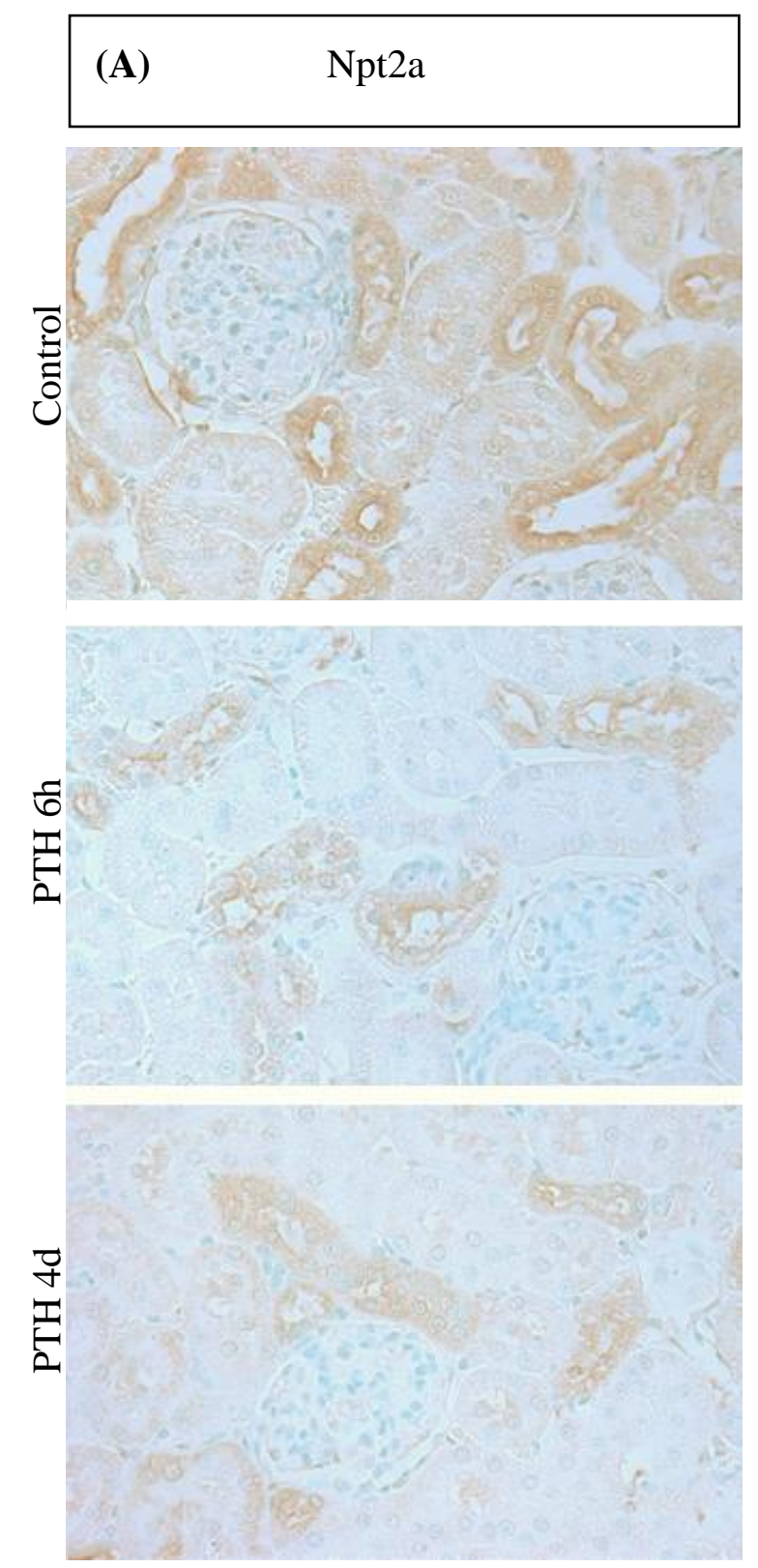

(B) $\quad \mathrm{Na}^{+} / \mathrm{K}^{+}$-ATPase $\alpha$-subunit
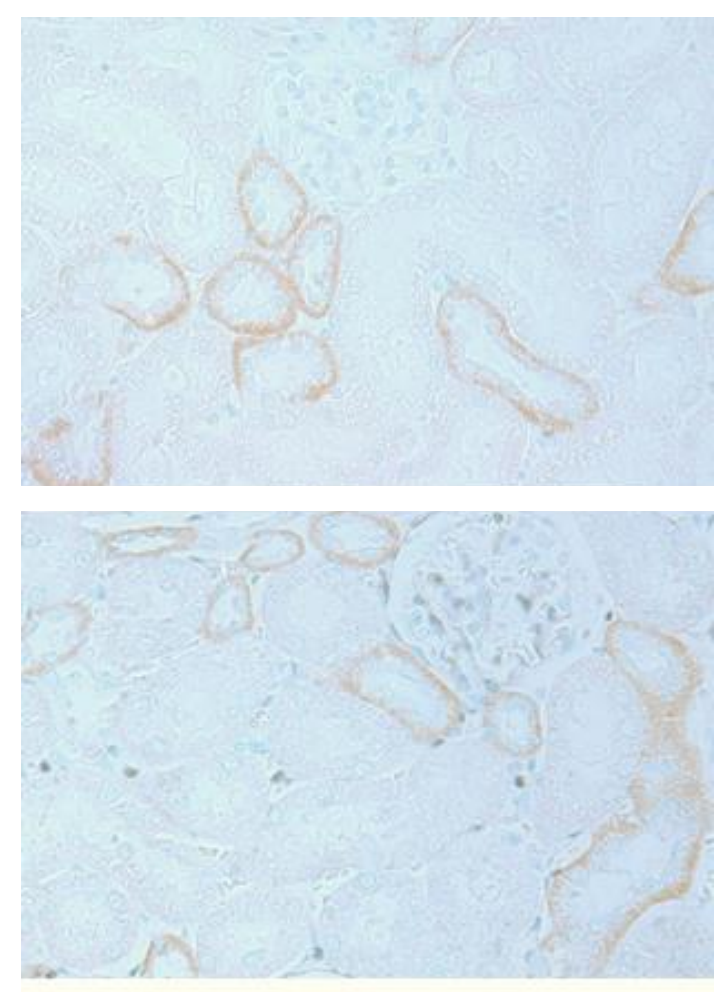

Figure 3: Effect of acute and chronic PTH on proximal tubule proteins. Fourmicrometer-thick sections of Sprague Dawley rat kidneys were prepared as described in Chapter IV Methods. Rats were treated with either vehicle, $6 \mathrm{~h} \mathrm{PTH}(5 \mu \mathrm{g} / \mathrm{kg}$ body weight), or $4 \mathrm{~d}$ PTH ( $5 \mu \mathrm{g} / \mathrm{kg}$ body weight, administered daily). Expression of (A) Npt2a and (B) the $\mathrm{Na}^{+} / \mathrm{K}^{+}$-ATPase $\alpha$-subunit was determined by immunohistochemistry, as described in Chapter IV Methods. Representative picture of control or PTH-treated kidney is shown $(\mathrm{n}=3)$. 


\section{FIGURE 4}
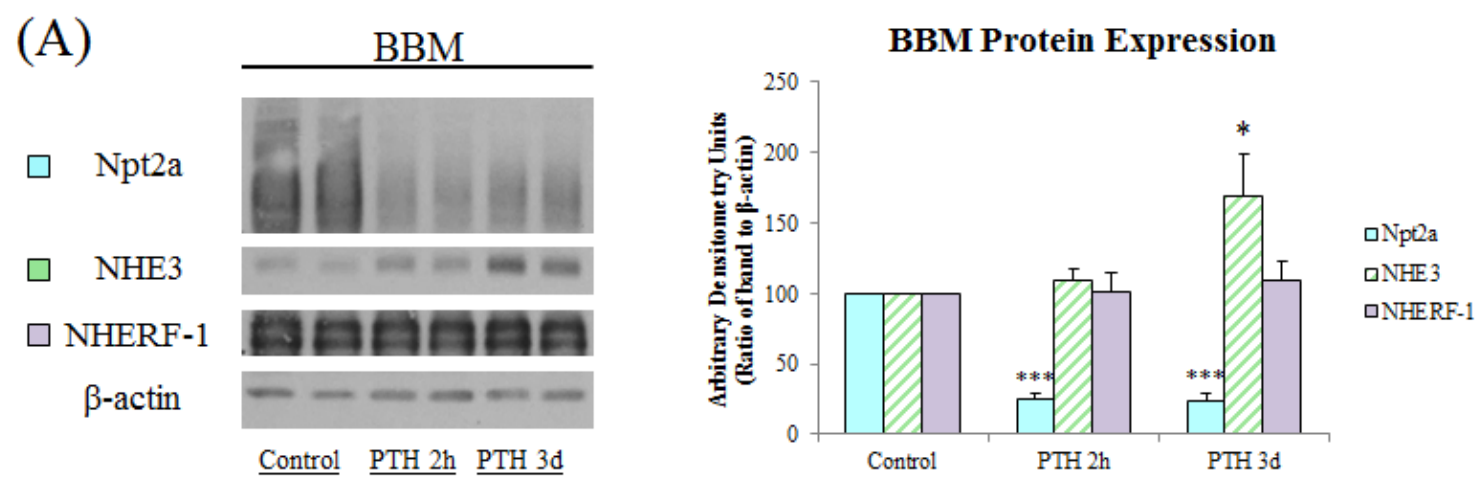

(B)

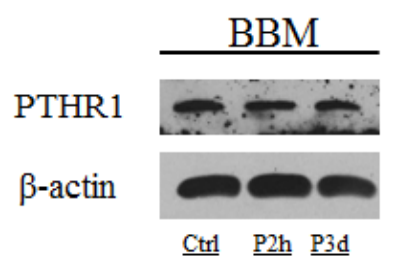

(C)

$\mathrm{Na}^{+} / \mathrm{K}^{+}$-ATPase $\alpha$-subunit
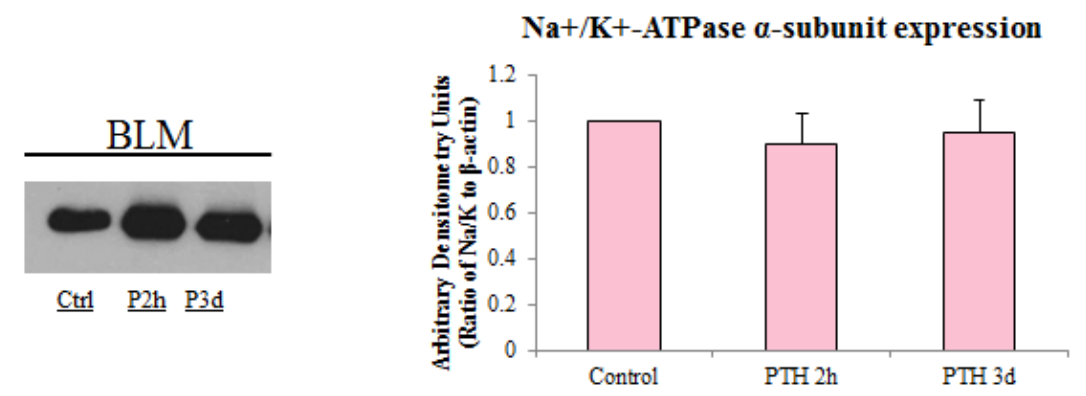

Figure 4: Effect of PTH on BBM and BLM protein expression in proximal tubule cells. (A) OK WT cells were treated with 100nM PTH(1-34) and brush-border membranes (BBM) were isolated as described in Chapter IV Methods. Proteins were separated by SDS-PAGE and visualized through chemiluminescence on X-ray film. Bands for Npt2a, NHE3, and NHERF-1 were normalized as a ratio of $\beta$-actin band. * and *** are indicative of $P<0.05$ and $P<0.001$, respectively, versus control, as determined by one-way ANOVA $(n=4)$. (B) Western blot showing expression of the PTHR1 in BBM of OK cells in response to PTH (n=3). (C) Western blot of $\mathrm{Na}^{+} / \mathrm{K}^{+}$-ATPase $\alpha$-subunit expression in OK cell BLM in response to PTH $(n=4)$. 
To address the potential mechanism of the chronic down-regulation of Npt2a by PTH, we next examined the effect of PTH on the mRNA expression of both Npt2a and NHE3. As shown in Figure 5A, NHE3 mRNA expression is unaffected by $6 \mathrm{~h}$ PTH, whereas Npt2a mRNA expression (Figure 5B) displayed a significant reduction at both the $6 \mathrm{~h}$ (acute) and $4 \mathrm{~d}$ (chronic) time points.

\section{FIGURE 5}

(A) NHE3 mRNA expression

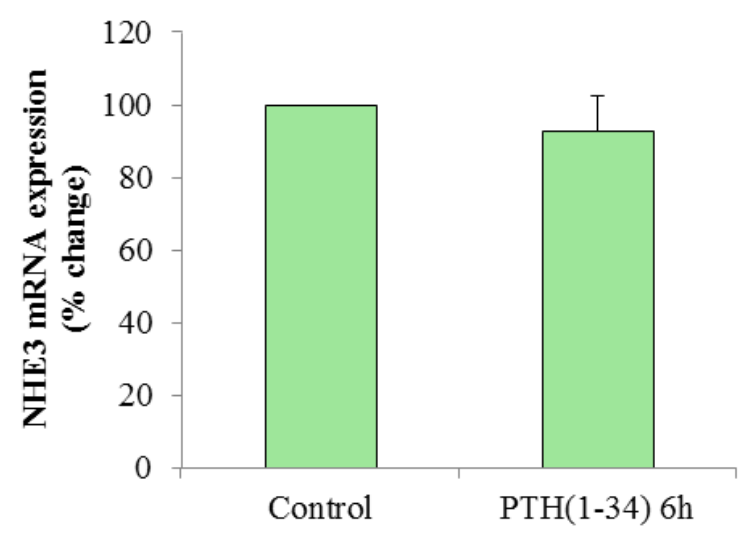

(B)

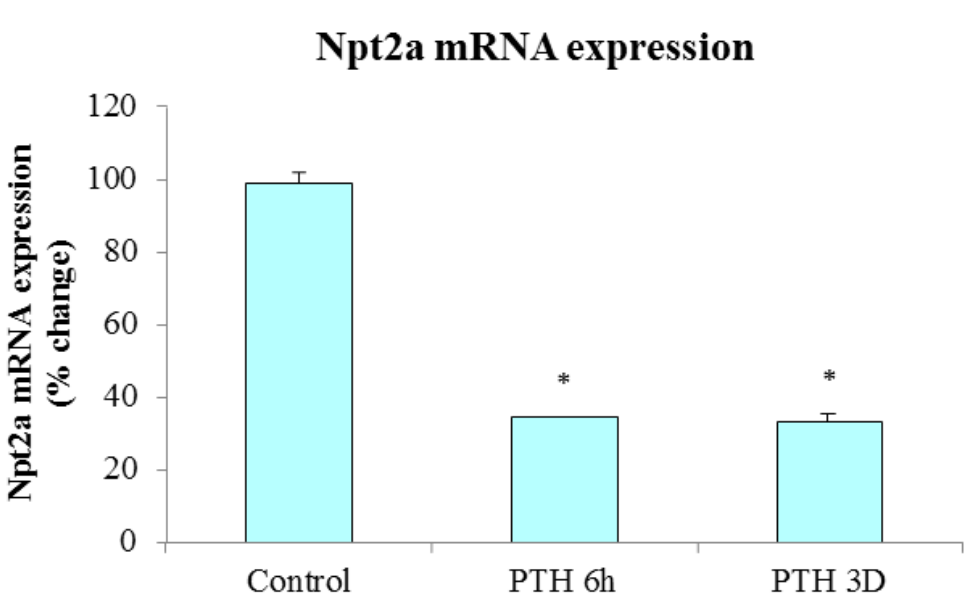

Figure 5: Effect of PTH on Npt2a and NHE3 mRNA expression. OK cells were treated for $6 \mathrm{~h}$ or $3 \mathrm{~d}$ with $100 \mathrm{nM}$ PTH. As described in Chapter IV Methods, RT-qPCR was performed to quantitate (A) NHE3 mRNA expression in response to PTH ( $\mathrm{n}=3$ ), or (B) Npt2a mRNA expression in response to PTH $(\mathrm{n}=4)$. * is indicative of $P<0.05$, as calculated by one-way ANOVA. 
We observed no change in NHE3 BBM expression with acute PTH, and an increase with chronic PTH. While PTH is classically known to inhibit sodium/hydrogen exchange at the apical membrane, Yang et al. demonstrated that acute PTH induces that downregulation not through endocytosis of the transporter but instead through the redistribution of NHE3 to the base of the microvilli, where it is less active ${ }^{84}$. We showed that neither acute nor chronic PTH has an effect on NHERF-1 BBM expression, consistent with work previously demonstrated by Weinman et al. ${ }^{32}$. Previous data reported by our lab demonstrated that acute PTH-mediated downregulation of the expression and activity of the sodium pump desensitizes with chronic $\mathrm{PTH}^{85}$. In OK cells, we observed a slight decrease in $\mathrm{Na}^{+} / \mathrm{K}^{+}$-ATPase expression after $2 \mathrm{~h}$ PTH treatment, although this was not significant. In rat renal cortex, we were unable to observe through IHC a significant decrease in $\mathrm{Na}^{+} / \mathrm{K}^{+}$-ATPase expression with acute PTH. However, $\mathrm{Na}^{+} / \mathrm{K}^{+}$-ATPase is endocytosed to sub-membrane compartments in response to PTH, and thus the apparent stability of $\mathrm{Na}^{+} / \mathrm{K}^{+}$-ATPase expression with $6 \mathrm{~h}$ and $4 \mathrm{~d}$ PTH may not accurately reflect the changes in distribution of the protein in response to $\mathrm{PTH}$.

The results of the qPCR studies suggest distinct mechanisms of transporter regulation by $\mathrm{PTH}$ in the proximal tubule. PTH acutely downregulates NHE3 through NHE3 phosphorylation and subsequent redistribution to the base of the microvilli ${ }^{84,86}$, and this effect appears to desensitize with chronic PTH. We observed no effect of PTH on NHE3 mRNA expression. In contrast to PTH-mediated NHE3 regulation, $\mathrm{PTH}$ induces a rapid reduction in Npt2a BBM expression through endocytosis and lysosomal degradation of the transporter, and this suppression of Npt2a expression is sustained with chronic PTH. Here we report preliminary evidence that Npt2a mRNA reduction is a 
potential mechanism for the acute and chronic hypophosphatemic effect of PTH. The experiments detailed within this chapter provide support for the idea that chronic regulation of Npt2a by PTH occurs at the mRNA level. The subsequent studies outlined within this dissertation build upon these findings, expand our current understanding of Npt2a regulation by PTH, and provide a basis for the further exploration and study of mechanisms underlying homeostatic control of serum phosphate. 


\title{
CHAPTER III \\ HYPOTHESIS AND SPECIFIC AIMS
}

\begin{abstract}
$\underline{\text { Overall Aim }}$
The work detailed within this dissertation elucidates three aspects of the mechanism by which parathyroid hormone regulates Npt2a mRNA within renal proximal tubules. Preliminary data addressing this physiological process has shown that PTH regulation of $\mathrm{Npt} 2 \mathrm{a}$ is unique, in that transcript levels of $\mathrm{Npt} 2 \mathrm{a}$ in addition to protein expression are decreased in response to hormone stimulation. Aim 1 determines whether PTH affects Npt2a mRNA at the transcriptional or post-transcriptional level. Aim 2 identifies the signaling pathways that contribute to PTH-mediated Npt2a mRNA downregulation. Lastly, Aim 3 characterizes the PTH-responsive phosphoproteome of the proximal tubule, with a focus on identifying PTH-responsive RNA-binding proteins and elucidating their role in PTH-mediated Npt2a mRNA downregulation.
\end{abstract}

\section{Hypothesis}

\section{PTH induces sustained suppression of Npt2a by decreasing Npt2a mRNA levels}

To address this hypothesis, this work will examine three aspects of the mechanism(s) involving PTH-mediated Npt2a mRNA downregulation. 


\section{Specific Aim I:}

\section{Determine whether PTH affects Npt2a mRNA at the transcriptional or post- transcriptional level}

Acute PTH treatment causes a host of protein expression changes in the proximal tubule, some of which persist with chronic PTH exposure while others desensitize. Whereas sodium/potassium pump and sodium/hydrogen exchange expression and activity experience a transient decrease in response to PTH, suppression of Npt2a persists with prolonged PTH. Npt2a promoter activity does not change with PTH. However, our data suggests the likely possibility that PTH regulates Npt2a transcript levels in addition to inducing Npt2a protein degradation. Therefore, we hypothesize that PTH decreases

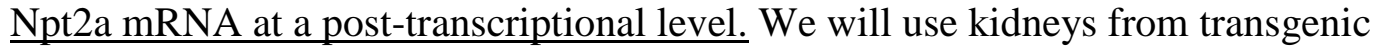
hyperparathyroid mice and opossum kidney cells to study the effects of PTH on Npt2a mRNA expression and stability.

\section{Specific Aim II:}

\section{Identify the second messenger pathway responsible for the PTH-mediated effect on Npt2a mRNA}

The PTH receptor is a type II G protein-coupled receptor (GPCR) capable of coupling with several different $\mathrm{G}$ proteins. The PKA and PKC signaling pathways are the two main second-messenger pathways activated upon PTH binding to the PTH receptor. Both pathways contribute to the regulation of Npt2a at the protein level, but it is unknown which of these pathways contributes to the PTH-mediated decrease in Npt2a mRNA. We hypothesize that activation of the PKA pathway is required for mediating the 
$\underline{\text { PTH-induced decrease in Npt2a mRNA. We will use the opossum kidney }(\mathrm{OK}) \text { cell }}$ culture line to study the effects of both the PKA and PKC pathways on Npt2a mRNA levels.

\section{Specific Aim III:}

\section{Characterize the PTH-responsive phosphoproteome in proximal tubule cells}

Based on the results of Aim 2, we determined that PTH produces Npt2a mRNA destabilization through both PKA- and PKC-dependent mechanisms. Therefore, we aim to characterize the PTH-responsive phosphoproteome in the OK cell, with a focus on identifying RNA-binding proteins that are regulated by PTH. We hypothesize that PTHmediated Npt2a mRNA destabilization is dependent on upregulated expression and/or phosphorylation of an RNA-binding protein. We will use a proteomic approach to identify possible protein mediators of the PTH response, focusing on differential phosphorylation of RNA-binding proteins between control and PTH-treated cells. 


\section{CHAPTER IV}

\section{SPECIFIC AIM I: PTH DECREASES NPT2A MRNA THROUGH POST-TRANSCRIPTIONAL MECHANISMS}

\section{$\underline{\text { Background }}$}

The story of parathyroid hormone and renal phosphate reabsorption is an old one, and yet an incomplete one. Decades of research dating back to the 1920 s detail the effects of PTH on proximal tubular reabsorption of the filtered load of phosphate ${ }^{87}$. The process by which PTH regulates Npt2a protein expression has been extremely well studied, and the kinetics of the hormone response have been determined down to the minute. In the bloodstream, circulating PTH has a half-life of approximately five minutes ${ }^{88}$, which suggests that under normal circumstances, the effects of PTH are rapid and short-lived. However, pathophysiological conditions such as primary hyperparathyroidism produce sustained elevated serum PTH levels that produce prolonged effects - most notably on serum phosphorus, calcium, and vitamin D levels. As previously discussed, the clinical manifestations of prolonged PTH stimulation show evidence for a mechanism of chronic down-regulation of proximal tubular phosphate reabsorption apart from the regulation of apical membrane protein expression of Npt2a. Considering the cellular energetics of constitutively transcribing, synthesizing, and trafficking Npt2a to the apical membrane, 
only to be immediately pulled back into the lysosomes and degraded, it is unlikely that PTH affects Npt2a expression solely at the protein level.

Preliminary data by our lab suggest that PTH is capable of regulating Npt2a at the mRNA level. This finding is novel, and opens the door to exploring actions of PTH on the proximal tubule previously unconsidered. Decreased mRNA expression of a gene can be produced by two general mechanisms: (1) decreased transcription, or (2) decreased mRNA stability. While limited studies on the effect of PTH on either of those mechanisms for Npt2a regulation have been performed, Npt2a has been shown to be regulated at the transcriptional and post-transcriptional level by several other factors.

\section{$\underline{\text { Npt2a gene expression }}$}

In discussing the potential mechanisms for regulation of Npt2a mRNA expression, it serves to examine elements of gene structure and function that are already known. The human Npt2a gene is present on Chromosome 5 and has been well characterized by several laboratories ${ }^{89}$. Both the human and the mouse Npt2a genes were initially cloned and characterized in 1996 by Hartmann et al. ${ }^{90}$ The human Npt2a gene is approximately $14 \mathrm{~kb}$ long, with 13 exons and 12 introns ${ }^{90,91}$. Within the gene promoter region are CAAT and TATA boxes that together with the sequence of exon 1 drive gene transcription. Additionally, within both the human and murine genes, the translation initiation site is found within exon $2^{90}$.

The promoter region of the Npt2a gene has several cis-acting elements that govern its transcription. The transcription initiation site was identified as an adenosine residue 57 base pairs upstream of the 3' end of the first exon (44 base pairs long), with 
the TATA box 28 bp still upstream from that ${ }^{91}$. Further upstream of the TATA box in the 5' flanking region of the Npt2a gene, there are several cis-acting elements, including a cAMP responsive element, as well as binding sites for the transcription factors AP-1, AP2, and $\mathrm{Sp}^{91}$. This 5 ' flanking region of the NpT2 gene also contains a Vitamin D Response Element, as well as elements responsible for kidney-specific gene $\operatorname{expression}^{91,92}$.

Npt2a promoter function is influenced by several factors. Basal rates of Npt2a transcription are affected by internal factors, including NHERF-1, as well as external factors such as vitamin A, vitamin D, and thyroid hormone. Basal levels of vitamin D appear to maintain juxtamedullary expression of $\mathrm{Npt} 2 \mathrm{a}$, as deficiency of vitamin $\mathrm{D}$ leads to decreased Npt2a mRNA and protein expression that is restored upon administration of vitamin D. In vitamin D-deprived rats, administration of vitamin D increases Npt2a gene promoter activity, leading to increased Npt2a mRNA and protein expression ${ }^{92}$. However, this is thought to be a weak effect, as increased vitamin D stimulation fails to elicit physiological effects in phosphate reabsorption beyond baseline uptake ${ }^{6}$. Vitamin A has also been shown to play an important role in Npt2a gene expression. Retinoic Acid Response Elements (RAREs) were identified within the promoter of the Npt2a gene by Masuda et al. ${ }^{93}$, who discovered that a deficiency in vitamin A induces increased renal phosphate excretion through down-regulation of both the Npt2a and Npt2c genes. NHERF-1, the regulatory protein important for Npt2a protein maturation and membrane localization, has also been shown to be important for the expression of the Npt2a gene, as demonstrated by Khundmiri et al., who showed that OK cells lacking NHERF-1 expressed less Npt2a mRNA than their WT counterparts, and that Npt2a transcript levels 
were restored upon transfection of NHERF-1-deficient cells with human NHERF-1 constructs $^{94}$. Lastly, thyroid hormone has also been identified as another factor that upregulates Npt2a gene expression by increasing its promoter activity. Ishiguro et al. ${ }^{95}$ identified thyroid hormone responsive elements (TRE) within intron 1 of the Npt2a gene, and showed that $\mathrm{T}_{3}$ upregulates $\mathrm{Npt} 2 \mathrm{a}$ gene expression in a renal cell-specific manner.

Conversely, there are also several factors known to downregulate Npt2a promoter function. Elevated levels of bicarbonate/carbon dioxide independent of $\mathrm{pH}$ were shown by Jehle et al. ${ }^{96}$ to decrease Npt2a promoter function, and thereby decrease protein expression and phosphate transport. Vitamin D, in addition to activating Npt2a promoter activity, can also indirectly decrease Npt2a gene transcription, as vitamin D also upregulates fibroblast growth factor 23 (FGF23), a phosphaturic hormone. FGF23 activates ERK1/2 signaling in the proximal tubule, and this results in phosphorylation of NHERF-1 with the subsequent endocytosis and degradation of $\mathrm{Npt}_{2} \mathrm{a}^{57}$. However, in a classic endocrine negative feedback loop, FGF23 decreases expression of $1 \alpha-$ hydroxylase, leading to decreased levels of 1,25-dihydroxyvitamin D and also decreased expression of $\mathrm{Npt} 2 \mathrm{a}^{55,62}$. Transgenic mice featuring overexpression of FGF23 present with increased serum FGF23 and Klotho levels, and, notably, decreased Npt2a mRNA $\operatorname{expression}^{97}$.

\section{$\underline{\text { Post-transcriptional regulation of Npt2a }}$}

While several studies have demonstrated mechanisms for robust regulation of Npt2a expression at the gene level, others also demonstrate unique mechanisms for regulating Npt2a expression at a post-transcriptional level. Factors that have been shown 
to modulate Npt2a mRNA stability include serum phosphate levels, glucocorticoids, and estrogen. Npt2a mRNA expression does not change in response to acute changes in dietary phosphate, but does however change in response to chronic changes in dietary phosphate ${ }^{98-100}$. Chronic low phosphate diets induce increased expression of Npt2a protein and mRNA in renal proximal tubules. Low serum phosphate induced by chronic low phosphate diets is accompanied by an increase in cytosolic calcium, and that increase has been shown to be critical for the upregulation of Npt2a transcript expression in response to low phosphate diets ${ }^{101}$. Tenenhouse et al. ${ }^{99}$ propose that this is due in part to post-transcriptional changes in Npt2a mRNA stability, as the increase in Npt2a protein expression produced by chronic low phosphate is much greater than the increase in $\mathrm{Npt} 2 \mathrm{a}$ mRNA expression. Later, Moz et al. ${ }^{3}$ supported this tenet when they showed that hypophosphatemia induces increased binding of cytosolic proteins to the 3' and 5' UTR of Npt2a mRNA, thus increasing stability and translation. Apart from regulation by serum phosphate, glucocorticoids decrease both $\mathrm{Npt} 2$ a protein and mRNA expression ${ }^{102-}$ ${ }^{104}$, the latter due to effects independent of promoter activity. Estrogen has been shown to downregulate Npt2a mRNA expression in a manner independent of the estrogen receptor alpha isoform $(\mathrm{ER} \alpha)^{5}$, potentially by decreasing transcript stability. Chronic Angiotensin II $^{105}$ and acute inflammation in response to LPS administration ${ }^{106}$ have also been shown to post-transcriptionally modulate Npt2a expression.

$\underline{\text { Evidence for a role of PTH in the regulation of Npt2a gene expression }}$

The vast majority of studies that have examined regulation of Npt2a by PTH have focused on regulation at the protein level. While it has been clearly demonstrated that 
apical protein expression of Npt2a is greatly diminished in response to PTH, few studies have examined whether regulation of the transporter also occurs at the mRNA level, and those that have studied the effect of PTH on Npt2a mRNA expression have been limited and inconclusive. Kilav et al. ${ }^{107}$ were unable to demonstrate an effect of elevated PTH on Npt2a mRNA in a rat model of diet-induced hyperparathyroidism. However, the diet was only sustained for three weeks, and thus may not have provided sufficient time to observe changes in transcript levels. Confounding systemic effects from the diet itself may have also counteracted any potential downregulatory actions of the elevated PTH itself. In contrast to Kilav's findings, three other laboratories have reported an effect of PTH on Npt2a mRNA. Kempson et al. ${ }^{81}$ showed that acute PTH treatment in parathyroidectomized rats decreased Npt2a mRNA expression by 30\%. Independently, Moe et al. ${ }^{108}$ demonstrated that rats with hyperparathyroidism secondary to chronic kidney disease display a 50\% reduction of levels of the Npt2a transcript. Friedlaender et al ${ }^{63}$ reported modest inhibitory effects of PTH on Npt2a mRNA expression in PTHinfused rats, which was augmented further when administered alongside vitamin D.

Decreased transcript levels can generally be produced by either decreased gene promoter activity or by decreased mRNA stability, and work done by Hilfiker suggests that the inhibitory effect of PTH on Npt2a mRNA may be due to the latter. In their studies on the Npt2a gene promoter, Hilfiker et al. ${ }^{109}$ showed that PTH has no inhibitory effect on Npt2a promoter function. However, they did not examine Npt2a mRNA expression in response to PTH. While some controversy exists as to whether PTH regulates Npt2a mRNA levels, our preliminary data show a significant inhibitory effect. Since PTH has been shown to have no effect on Npt2a gene promoter activity, changes in 
Npt2a mRNA levels in response to PTH most likely occur at the post-transcriptional level. Our preliminary data, as well as the aforementioned studies on Npt2a mRNA expression in response to PTH, indicate PTH is capable of regulating Npt2a beyond the protein level. What has not been defined is the mechanism(s) for the PTH-mediated decrease in Npt2a mRNA. Based on our preliminary data and literature review, we hypothesize that PTH decreases Npt2a mRNA at a post-transcriptional level. The studies outlined in this chapter build upon our novel finding that Npt2a mRNA expression is controlled by PTH. 


\section{$\underline{\text { METHODS AND MATERIALS }}$}

Materials: Bovine PTH (1-34) was purchased from Bachem Biosciences Inc. (King of Prussia, PA). Antibody against mouse Npt2a was a generous gift from Dr. Moshe Levi (University of Colorado). Opossum Npt2a antibody was developed as described previously ${ }^{110}$. NHERF-1 antibody was a gift from Dr. Weinman (University of Maryland $)^{76}$. HRP-conjugated $\beta$-actin antibody was purchased from Santa Cruz Biotechnology (Santa Cruz, CA). PTH receptor antibody was purchased from Covance (Princeton, NJ). Monoclonal antibodies against $\mathrm{Na}^{+}, \mathrm{K}^{+}$ATPase $\alpha_{1}$-subunit were purchased from Sigma-RBI (Natick, MA). Applied Biosystems PCR TaqMan PreDeveloped Assay Reagents and Ambion MirVana miRNA isolation kits were purchased from Life Technologies (Grand Island, NY). Immunohistochemistry reagents were purchased from Vector Laboratories (Burlingame, CA). Unless otherwise specified, all other chemicals were purchased from Sigma-Aldrich (St. Louis, MO).

PTH-D1 mouse model: The PTH-D1 mouse is a transgenic mouse model of primary hyperparathyroidism, wherein the mouse develops gradual hyperplasia of the parathyroid glands due to parathyroid-specific cyclin D1 overexpression. In response to the parathyroid hyperplasia, these mice gradually develop biochemical and histological features of primary hyperparathyroidism, including elevated PTH, high serum calcium, and low serum phosphorus ${ }^{111,112}$. PTH-D1 kidneys were either frozen in liquid nitrogen for later analysis by qPCR and Western blot, or were fixed overnight in paraformaldehyde and embedded in paraffin for analysis by immunohistochemistry. 
Kidneys from control mice of the same background strain as the PTH-D1 mice (FVB) and similar age were used for immunohistochemical and Western blot analyses. Control mRNA from 8-month-old FVB mice for qPCR experiments was a generous gift from Dr. Paul Epstein, University of Louisville.

Cell culture: Studies involving time-dependent effects on Npt2a mRNA stability were conducted using the opossum kidney $(\mathrm{OK})$ cell line, a continuous renal proximal tubule cell culture model derived from the Virginia opossum ${ }^{113}$. Importantly, the OK cell expresses PTH-responsive proteins, including the PTH receptor (PTHR1), coupled to $\mathrm{G}_{\mathrm{s}}$ and $\mathrm{G}_{\mathrm{q}} \cdot \mathrm{G}_{\mathrm{s}}$ and $\mathrm{G}_{\mathrm{q}}$ activate PKA and PKC, respectively, resulting in downregulation of Npt2a expression ${ }^{114}$. OK cells were cultured in Eagle's Modified Essential Medium supplemented with $10 \%$ fetal bovine serum and $1 \%$ penicillin/streptomycin in a humidified incubator at $37^{\circ} \mathrm{C}$ and $5 \% \mathrm{CO}_{2}$. Unless otherwise noted, cells were grown to 90-95\% confluence on 6-well plastic plates (Corning, Tewksbury, MA) and serumstarved overnight prior to treatment.

Immunohistochemistry: Immunohistochemical staining of PTH-D1 and control FVB kidneys was performed for Npt2a, $\mathrm{Na}^{+}-\mathrm{K}^{+}$ATPase $\alpha$-subunit, NHERF-1, and the PTH receptor. Four- $\mu$ m-thick sections were shaved from PTH-D1 and FVB kidneys fixed in paraffin blocks using a Leica RM2125RT microtome, and mounted on glass slides (Fisher, Pittsburgh, PA). Paraffin was cleared from slides by three washes with xylene (all washing steps were performed for five minutes unless otherwise noted). Kidneys were rehydrated by ethanol gradient $(100 \%, 95 \%, 70 \%)$ followed by two five-minute 
water incubations and a final Tween-Tris-Buffered Saline (TTBS) wash. Slides were incubated in a low-pH citrate buffer solution (Vector Labs) for 20 minutes in a $95^{\circ} \mathrm{C}$ water bath to retrieve the antigen. Following a 20-minute cooling period at room temperature, slides were washed three times in water. Endogenous peroxidase was quenched with $3 \% \mathrm{H}_{2} \mathrm{O}_{2}$, followed by three more washes with water and one wash with TTBS. Slides were incubated in $2.5 \%$ horse serum in TTBS for 30 minutes at room temperature to block non-specific binding. Sections were then incubated overnight at $4{ }^{\circ} \mathrm{C}$ with primary antibodies against Npt2a (1:5000), $\mathrm{Na}^{+}-\mathrm{K}^{+}$ATPase $\alpha$-subunit (1:2000), NHERF-1 (1:1000), or PTHR1 (1:200), diluted with 1\% BSA, 0.5\% Triton X-100, and $0.5 \%$ Tween-20 in water. Following primary antibody incubation, slides were washed three times with TTBS and incubated with biotin-conjugated secondary antibodies for 30 minutes at room temperature. Slides were again washed three times with TTBS, incubated at room temperature for 30 minutes with Vectastain $\mathrm{ABC}$ reagent (Vector Labs), an avidin-biotin enzyme complex, followed by three more washes with TTBS. Sections were incubated with 3,3'-Diaminobenzidine (DAB, prepared according to Vector protocol) and monitored under a microscope. Following visualization of chromatographic tubule staining, slides were quenched in water to stop the colorimetric reaction, and counterstained with methyl green. Digital Images were obtained with a $\mathrm{Q}$ Color 5 camera attached to an Olympus BX51 microscope using Image pro software. Immunostaining was quantified with Image-Pro 6.2 software (Media Cybernetics, Silver Spring, MD). Five visual fields of cortex per kidney section were randomly selected and captured with a 40x objective. Positive DAB staining was defined in a color profile by selecting a range of $\mathrm{DAB}$ staining intensities and the profile was 
applied to all images collected. Values for total staining area/field were obtained and presented as sum of staining area for each group.

Brush Border Membrane (BBM) and Crude Membrane (CM) isolation: Brush-border membranes were isolated from OK WT cells grown on $0.1 \mu \mathrm{M}$ transwell filters using the $\mathrm{MgCl}_{2}$ precipitation method. Following PTH or actinomycin D treatment, cells were washed twice with ice-cold PBS and scraped into $2 \mathrm{~mL}$ tubes with $5 \mathrm{mM}$ Tris $50 \mathrm{mM}$ mannitol buffer $\mathrm{pH}$ 7.4. Cells were subjected to high-speed (20,500 rpm) homogenization with a PowerGen 125 polytron homogenizer (Fisher Scientific) for three 30-second intervals with an interval of 30 seconds between each stroke. Following homogenization, $\mathrm{MgCl}_{2}$ was added to the homogenate to a final concentration of $10 \mathrm{mM}$ and incubated on ice for 20 minutes with periodic vortexing. The homogenate was spun at $2000 \mathrm{rpm}$ for 5 minutes at $4^{\circ} \mathrm{C}$, and the pellet was discarded. The resulting supernatant was spun at $18,000 \mathrm{rpm}$ for 45 minutes at $4^{\circ} \mathrm{C}$ to pellet brush-border membranes. The pellet was resuspended in $5 \mathrm{mM}$ Tris $300 \mathrm{mM}$ mannitol buffer $\mathrm{pH}$ 7.4. For crude membrane isolation from PTH-D1 and FVB mouse kidneys, minced kidney cortex was first homogenized in a glass Teflon homogenizer with ten complete strokes. The homogenate was then subjected to high-speed homogenization as described above, without $\mathrm{MgCl}_{2}$ precipitation, followed by a low-speed and a high-speed spin.

Protein determination: Protein concentration of BBM and CM preparations was determined using a bicinchoninic acid (BCA) protein kit (Sigma) using bovine serum albumin (BSA) as a standard. 
Western blot analysis: Following protein estimation, equal amounts of membrane proteins were heated in Laemmli buffer at $95^{\circ} \mathrm{C}$ for 5 minutes to denature proteins. Protein samples were separated on a 10\% acrylamide gel by SDS-PAGE, and then transferred to a nitrocellulose membrane for immunodetection. Nitrocellulose blots were incubated in TTBS containing 5\% milk at room temperature for 1 hour in order to block nonspecific binding to the membrane. Membranes were then incubated with primary antibodies diluted in 5\% milk in TTBS overnight at $4^{\circ} \mathrm{C}$ on a rocker. Following overnight incubation, membranes were washed with TTBS three times for five minutes and once for 15 minutes. Membranes were then incubated in HRP-conjugated secondary antibodies diluted in 5\% milk in TTBS for 1 hour at room temperature, followed by another round of TTBS washing. Bands were detected by chemiluminescence (Pierce), and visualized on X-ray film.

Isolation of total RNA: OK cells were processed to isolate total RNA according to the manufacturer's instructions (MirVana miRNA isolation kit, Life Technologies, Grand Island, NY). RNA concentrations were quantified at $260 \mathrm{~nm}$ wavelength with $280 \mathrm{~nm}$ wavelength as reference. For isolation of RNA from mice, kidney cortex from PTH-D1 mice was dissected from decapsulated kidneys and homogenized with a pestle in MirVana lysis buffer. Once homogenized, total RNA was isolated as described above.

Quantitative real-time PCR: $1 \mu \mathrm{g}$ of RNA was reverse-transcribed to cDNA using a BioRad MyCycler thermal cycler with the High Capacity RNA-to-cDNA Master Mix system (Applied Biosystems, Foster City, CA) in a reaction volume of $25 \mu \mathrm{L}$. Reverse 
transcription was carried out at $25^{\circ} \mathrm{C}$ for $5 \mathrm{~min}, 42^{\circ} \mathrm{C}$ for $45 \mathrm{~min}$, and heat inactivated at $85^{\circ} \mathrm{C}$ for 5 min. Resulting cDNA was diluted four-fold prior to PCR, to a final volume of $100 \mu \mathrm{L} .5 \mu \mathrm{L}$ of diluted cDNA was PCR-amplified using FAM detection and TaqMan Gene expression system with $50 \mathrm{nM}$ Npt2a primers. 18S rRNA was used as a control house-keeping gene reference. For OK cells, Npt2a forward primer sequence was 5'TCTGAGAGTGCTGATGTACCTAAGT-3', and reverse primer sequence was 5'AGGTACTCATCCAACACCAGGTAT-3'. Real-time PCR was then conducted in an Applied Biosystems 7500 Real Time PCR System with thermocycling setting of $50^{\circ} \mathrm{C}$ for $2 \mathrm{~min}, 95^{\circ} \mathrm{C}$ for $10 \mathrm{~min}$, and 40 repeats of $95^{\circ} \mathrm{C}$ for $15 \mathrm{sec}$ followed by $60^{\circ} \mathrm{C}$ for $1 \mathrm{~min}$. Relative quantitation was achieved by normalizing to $18 \mathrm{~S}$ rRNA reference values, as detected through FAM-MGB, and by calibrating to control cells using the $2^{-\Delta \Delta C t}$ method as described in Applied Biosystems User Bulletin 2: Rev B. For mouse RNA samples, the $\Delta \mathrm{C}_{\mathrm{T}}$ value was determined using $18 \mathrm{~S}$ and $\Delta \Delta \mathrm{C}_{\mathrm{T}}$ values determined using the $\Delta \mathrm{C}_{\mathrm{T}}$ value from one FVB control as the calibrator for all samples (FVB and PTHD1). For OK cell time course, the Npt2a mRNA levels were expressed relative to control $(t=0)$ that was set to 100 for each experiment. The average values from 4-6 independent experiments $+/-$ SEM were plotted on a semilogarithmic scale and the half-life calculated by linear regression.

Luciferase reporter gene assays: Npt2a promoter-luciferase reporter gene constructs (Npt2a-luc) containing -208 bp and -4700 bp were a generous gift of Heine Murer ${ }^{115}$. Npt2a-luc or pGL3-basic and Renilla luciferase reporter (pRL-TK, Promega) plasmids were cotransfected into OK-WT cells using Lipofectamine 2000 (Invitrogen). At $24 \mathrm{~h}$ 
post-transfection, firefly luciferase and Renilla luciferase activities were determined using Dual-Glo Luciferase Assay System (Promega) according to the manufacturer's protocol. The Luciferase:Renilla ratios were determined per well. For each independent experiment the cells were transfected in triplicate, and the values averaged.

Statistics: Data are shown as mean \pm SE. The $n$ values represent the number of independent experiments. Each experiment was performed in triplicate unless otherwise indicated. $P$ values were calculated by Student's $t$-test or by one-way or two-way ANOVA, followed by Bonferroni analysis using GraphPad Prism software. A $P$ value $<0.05$ was a priori considered statistically significant.

Ethical aspects of the proposed research: All animal studies in this dissertation were performed in accordance with the University of Louisville IACUC. Radiation experiments throughout this dissertation were performed under the auspices of the University of Louisville Radiation Safety Office. We have reviewed the literature and concluded that our proposed research does not needlessly duplicate previous studies. We have examined every aspect of our research plan for biohazards and have made all the appropriate arrangements to ensure that no one's health is damaged in performing these experiments 


\section{$\underline{\text { RESULTS }}$}

\section{Part 1: Effect of chronic primary hyperparathyroidism on renal tubular proteins}

To examine the effect of PTH on Npt2a mRNA expression in vivo, studies were conducted using a transgenic mouse model of chronic primary hyperparathyroidism, the PTH-D1 mouse. This model was developed and characterized by Andrew Arnold's laboratory, and features parathyroid-specific cyclin D1 overexpression, leading to the gradual development of primary hyperparathyroidism ${ }^{111,112}$. The 8-month-old PTH-D1 model of chronic hyperparathyroidism is advantageous due to the animal's endogenous over-production of PTH, eliminating the need for invasive thyroparathyroidectomy surgery and administration of exogenous PTH, thus relieving stress on the animal and mimicking more closely the human condition of primary hyperparathyroidism. Consistent with the clinical features of primary hyperparathyroidism, these animals develop elevated serum $\mathrm{PTH}$ and $\mathrm{Ca}^{2+}$ and decreased serum $\mathrm{P}_{\mathrm{i}}^{111}$.

IHC using kidneys from control and PTH-D1 mice confirmed that the PTH-D1 mice display chronically reduced expression of $\mathrm{Npt} 2 \mathrm{a}$ in the proximal tubule in comparison to age-matched control mice (Figure 6A and 6E); the expression of the $\mathrm{Na}^{+} / \mathrm{K}^{+}$-ATPase $\alpha_{1}$ subunit, PTHR1, and NHERF-1, however, did not differ (Figures 6B

-6D) between the two animal models. Crude membranes isolated from kidney cortex of FVB and PTH-D1 mice also showed no expression differences in the $\mathrm{Na}^{+} / \mathrm{K}^{+} \alpha_{1}$ subunit or NHERF-1 (Figure 6F). 
FIGURE 6

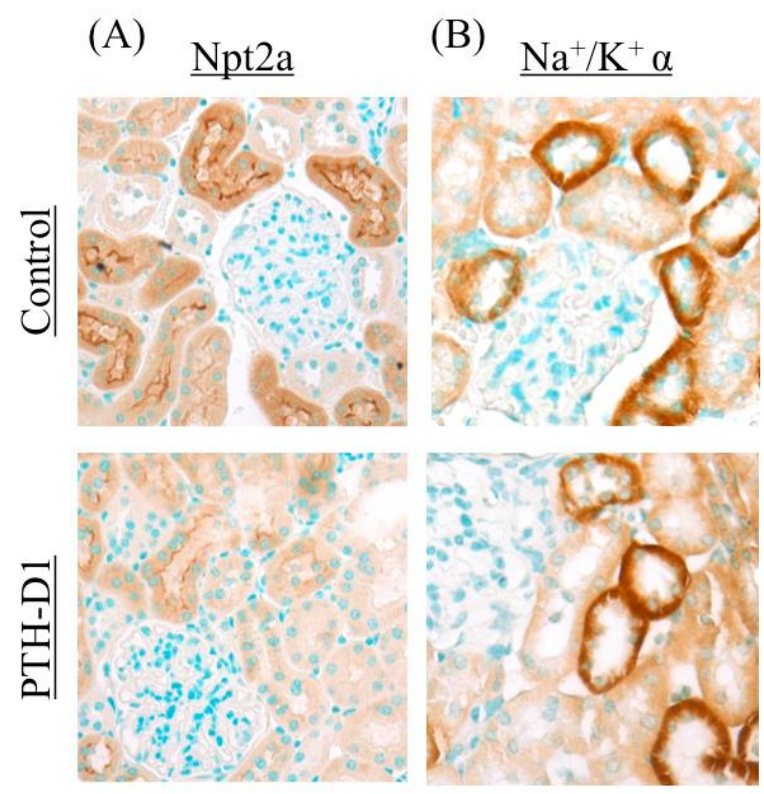

(C) NHERF-1

(D)

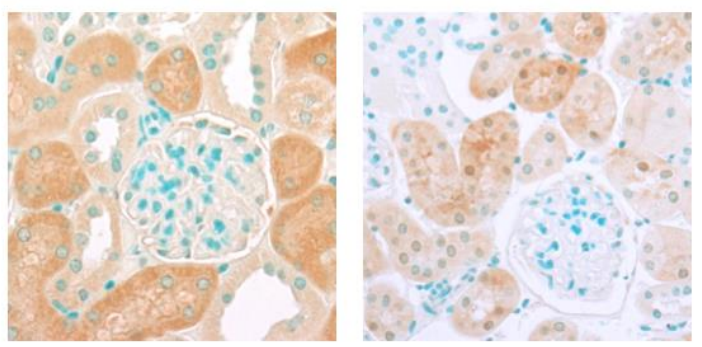

(E) Npt2a IHC Quantitation

(F)
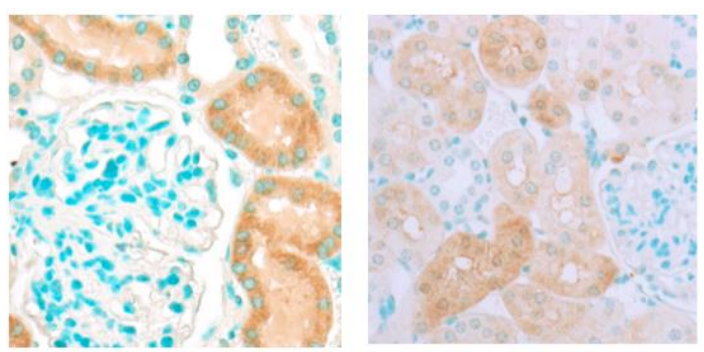

Crude membrane protein expression
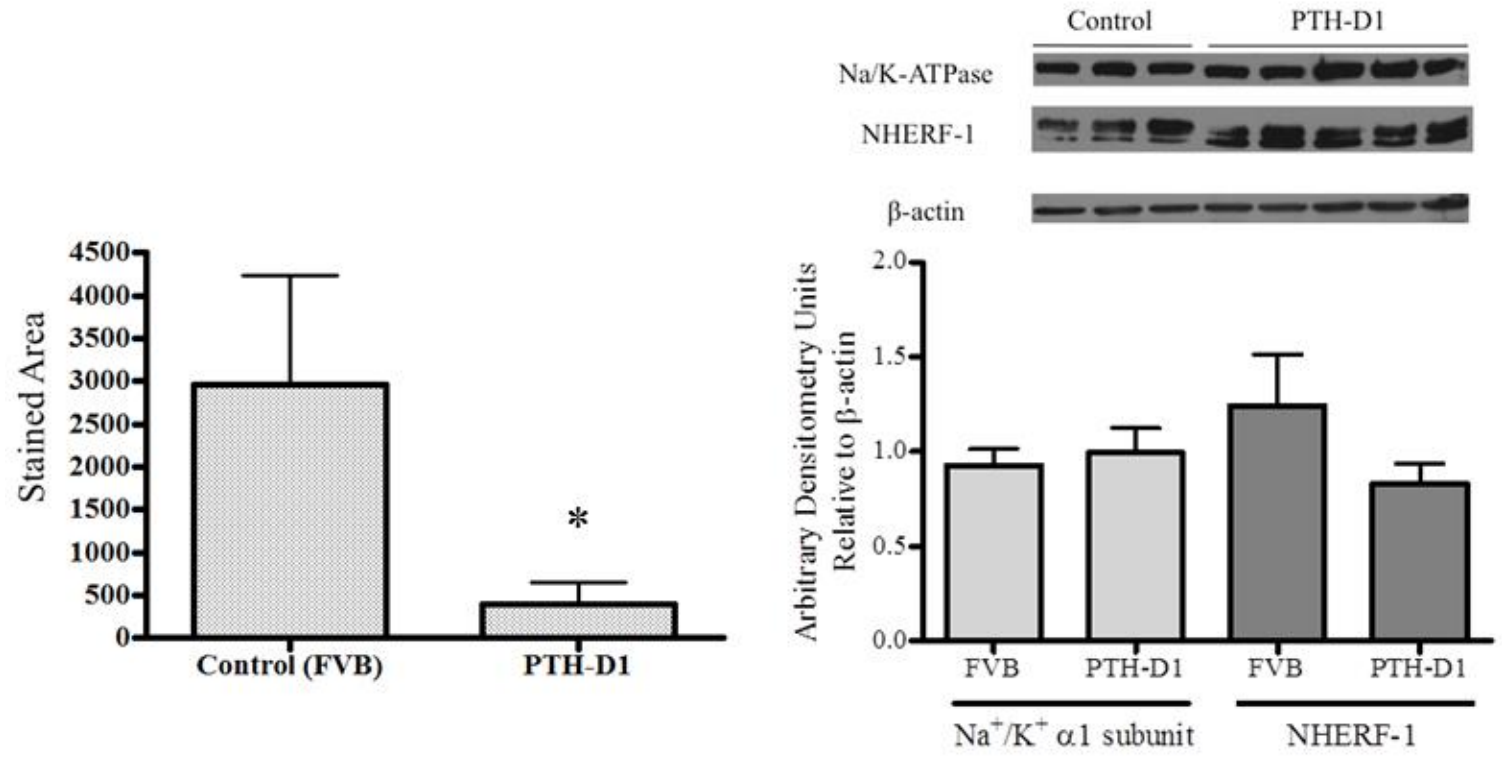

Figure 6: Proximal tubule protein expression in response to chronic PTH.

Immunohistochemical staining for (A) Npt2a, (B) $\mathrm{Na}^{+} / \mathrm{K}^{+}$-ATPase $\alpha_{1}$-subunit, (C) NHERF-1, and (D) PTHR1 in control FVB and PTH-D1 mouse kidneys. (E) Quantitation of Npt2a IHC staining from (A). (F) Crude membranes were prepared from cortex of FVB and PTH-D1 kidneys and Western blot analyses performed for NHERF-1 and $\mathrm{Na}^{+} / \mathrm{K}^{+}$ATPase $\alpha_{1}$-subunit expression. Images shown are representative $(\mathrm{n}=6) . *$ is indicative of $P<0.05$, as determined by student's t-test. 
Renal proximal tubule Npt2a expression analysis of the PTH-D1 mouse is consistent with the biochemical characterization performed by Imanishi et al. ${ }^{11}$, confirming the utility of this mouse model for exploring mechanisms of chronic phosphate regulation by PTH. To address the mechanism for the chronic down-regulation of Npt2a by PTH, Npt2a mRNA expression in control versus PTH-D1 mice was examined. As shown in Figure 7, PTH-D1 mice express Npt2a mRNA levels approximately $50 \%$ less than that of age-matched control mice.

\section{FIGURE 7}

\section{Npt2a mRNA expression in PTH-D1 mice}

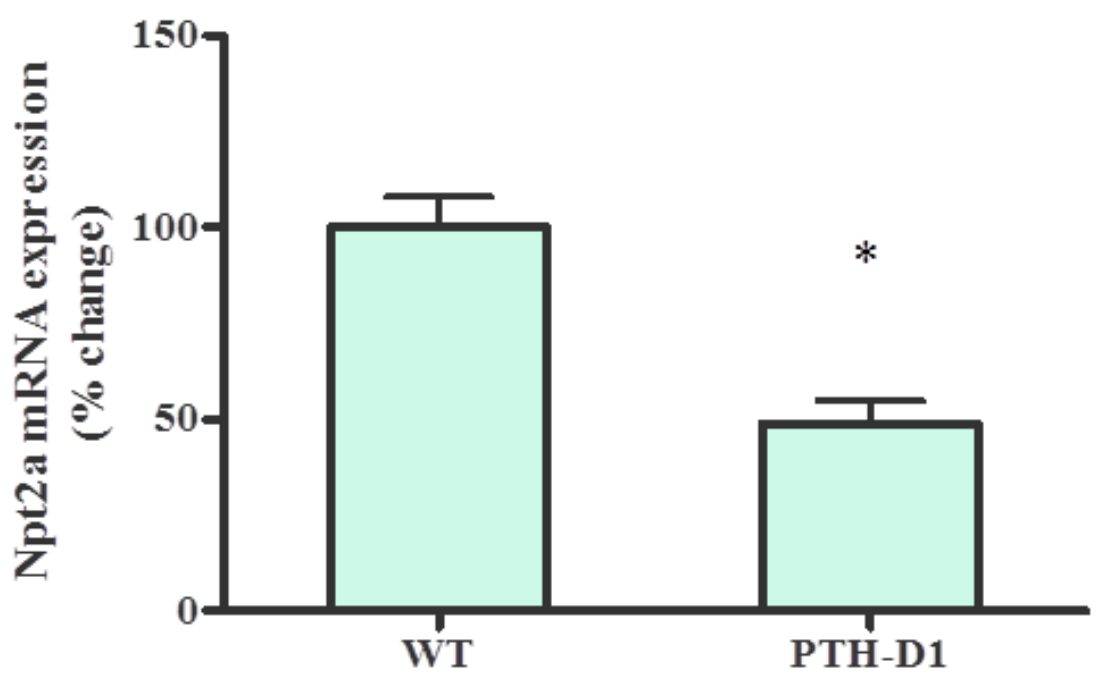

Figure 7: Npt2a mRNA expression in PTH-D1 mice. RT-qPCR with mRNA isolated from control and PTH-D1 kidneys (as described in Methods) revealed an approximate $50 \%$ decrease in Npt2a in hyperparathyroid PTH-D1 kidneys as compared to agematched control animals. ${ }^{*}$ is indicative of $P<0.05$. 


\section{$\underline{\text { Part 2: Time-dependent effects of PTH on Npt2a mRNA }}$}

To further explore the effect of PTH to Npt2a mRNA levels, the opossum kidney cell culture model, a continuous cell line derived from the Virginia opossum, was used to study time-dependent effects of PTH on Npt2a mRNA levels. OK cells were treated with 100nM PTH (1-34) from 30 minutes up to 24 hours. RNA was isolated as described in Methods, and reverse-transcribed into cDNA. Quantitative PCR was performed to measure Npt2a mRNA expression. PCR showed that PTH treatment caused a very rapid decrease in Npt2a mRNA, with an approximate $30 \%$ reduction observed after 30 minutes of PTH, reaching statistical significance at the $1 \mathrm{~h}$ time point (Figure 8). Npt2a mRNA levels continued to decrease in response to PTH in a time-dependent manner, with an ultimate reduction of approximately $75 \%$ achieved by $24 \mathrm{~h}$ PTH. As calculated by linear regression, the level of Npt2a mRNA fell by $50 \%$ after 2.2 hours of PTH treatment.

FIGURE 8

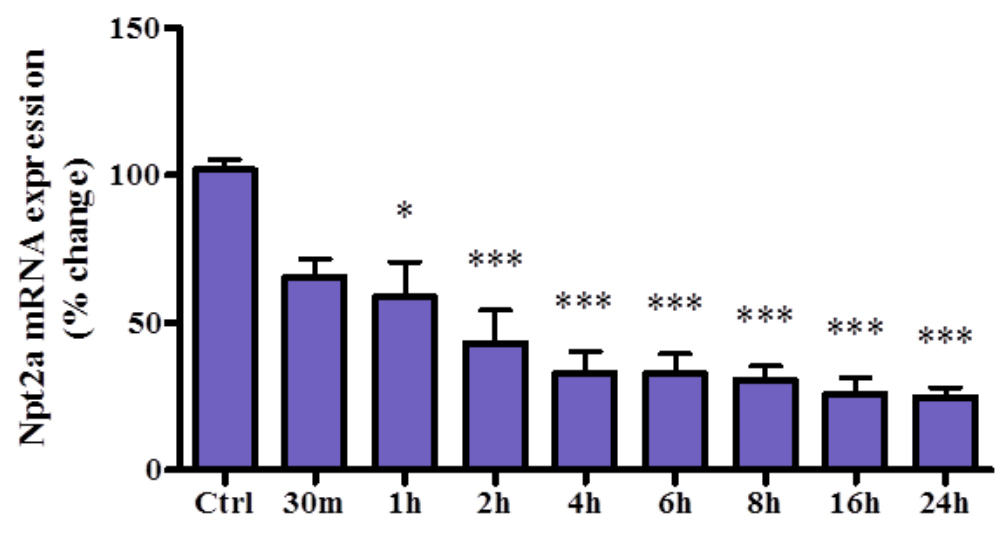

Figure 8: Npt2a mRNA expression in response to PTH in OK cells. RT-qPCR with mRNA isolated from OK WT cells treated with 100nM PTH (1-34) for times indicated. *, $* *$, and $* * *$ are indicative of $\mathrm{P}<0.05,0.01$, and 0.001 , respectively, as calculated by oneway ANOVA. 


\section{Part 3: Roles of transcription and translation in Npt2a mRNA stability}

To compare the stability of Npt2a mRNA under basal conditions and after treatment with PTH, OK cells were treated with actinomycin D, an inhibitor of transcription, over the course of 12 hours. Npt2a mRNA displayed a time-dependent decrease (Figure 9A), which achieved significance by the 4-hour time point, and an ultimate reduction of approximately $60 \%$ was observed by the 12 -hour time point. Under conditions of halted transcription, Npt2a mRNA displayed a $50 \%$ reduction after $8.6 \mathrm{~h}$, which is substantially longer than the amount of time required to achieve a $50 \%$ reduction in Npt2a mRNA during PTH treatment. To determine if the inhibitory effect of PTH on Npt2a mRNA expression involved transcription, OK cells were next pretreated with actinomycin $\mathrm{D}$ alone for 30 minutes, followed by combined treatment with actinomycin D and PTH. As shown in Figure 9B, the time-dependent decrease in Npt2a mRNA was blunted, only showing a significant decrease by the 4-hour time point. Treatment of OK cells with PTH while blocking transcription produced a curve very similar to that of actinomycin D alone, and was significantly different from the curve produced by PTH alone (Figure 9C). At the protein level, actinomycin D alone had little effect on brushborder membrane expression of Npt2a (Figure 9D). However, inhibiting transcription while treating OK cells with PTH did not prevent PTH-mediated endocytosis and degradation of Npt2a from the apical membrane, indicating that PTH-induced Npt2a protein degradation and PTH-induced Npt2a mRNA degradation are mechanistically independent events. 
(A) Npt2a mRNA expression following
treatment with actinomycin D

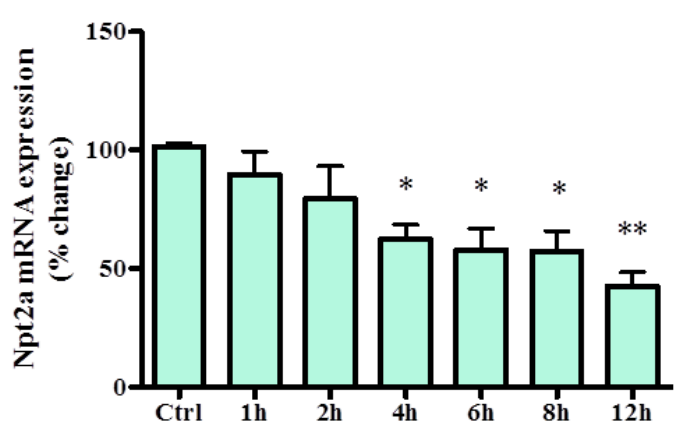

(C)

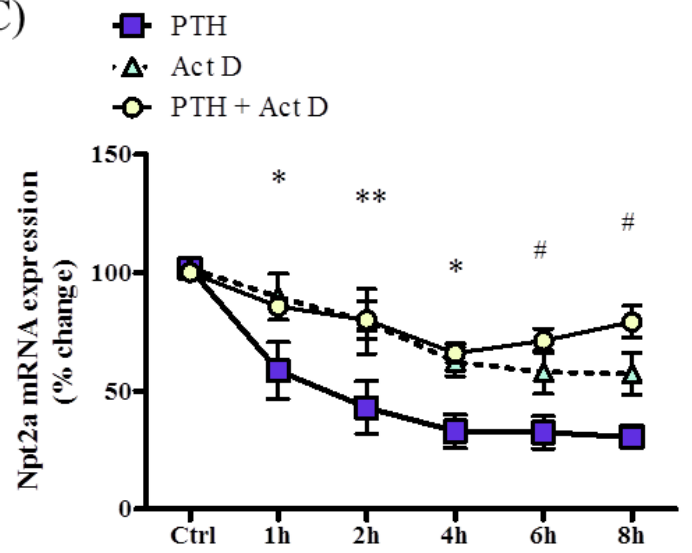

(B)

Npt2a mRNA expression with combined PTH and actinomycin D treatment

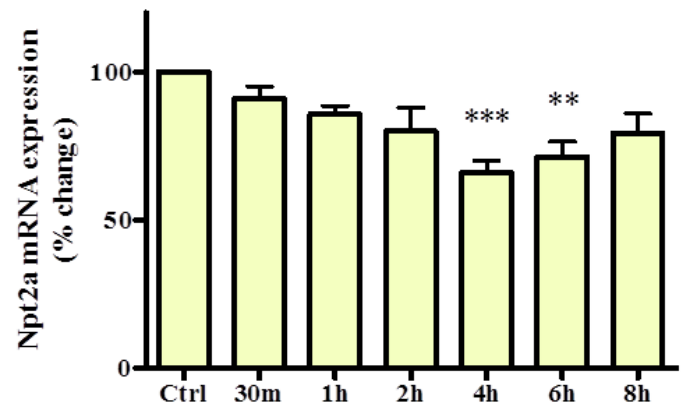

(D)
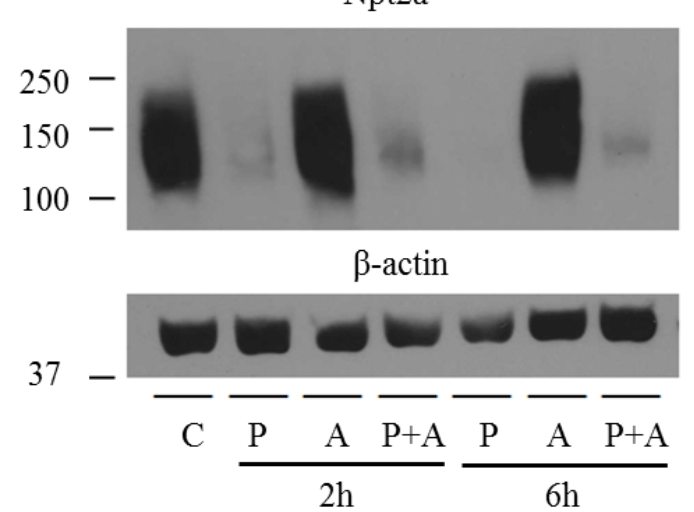

Figure 9: Role of transcription in Npt2a mRNA regulation. RT-qPCR with mRNA isolated from OK WT cells treated with (A) Actinomycin D (Act D, $1 \mu \mathrm{g} / \mathrm{mL}$ ), or (B) combined PTH and Act D (with 30min Act D pretreatment), for times indicated. *, **, and $* * *$ are indicative of $\mathrm{P}<0.05,0.01$, and 0.001 , respectively, as calculated by oneway ANOVA. (C) Comparison of time courses with PTH alone, Act D alone, and combined PTH + Act D treatment. * and ** are indicative of $P<0.05$ and 0.01 , respectively, between both Act D alone and PTH + Act D versus control. \# is indicative of $P<0.05$ for PTH + Act D versus control, as calculated by two-way ANOVA. (D) Western blot shows Npt2a BBM expression in response to PTH alone [P], Act D alone [A], or $\mathrm{PTH}$ and Act D combined $[\mathrm{A}+\mathrm{P}]$. Representative image shown $(\mathrm{n}=3)$. 
Similar to the previous experiment of inhibiting transcription in the presence of PTH, OK cells were next treated with cycloheximide, an inhibitor of translation, in the presence or absence of PTH. As shown in Figure 10, the inhibitory effect of PTH on Npt2a mRNA was blocked by cycloheximide treatment. Following $2 \mathrm{~h}$ of combined PTH and cycloheximide treatment, Npt2a mRNA expression was only reduced by approximately $25 \%$. This preservation of Npt2a mRNA in the presence of both cycloheximide and PTH was maintained after $6 \mathrm{~h}$ of combined treatment, in which Npt2a mRNA expression was significantly higher than in cells treated with PTH for $6 \mathrm{~h}$.

FIGURE 10

\section{Npt2a mRNA expression with cycloheximide treatment $+/-$ PTH}

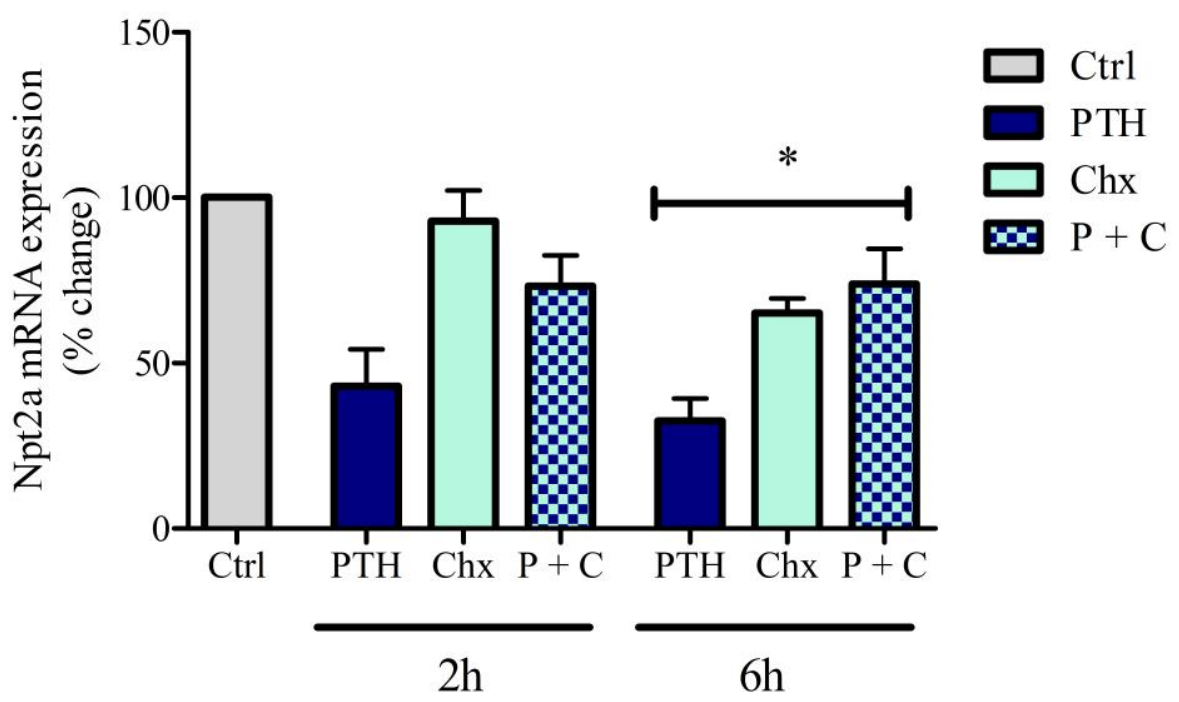

Figure 10: Role of translation in Npt2a mRNA regulation. OK cells were treated with cycloheximide (Chx, $1 \mu \mathrm{g} / \mathrm{mL}$ ) in the presence or absence of PTH. Cells treated with cycloheximide alone $(\mathrm{Chx})$ or both cycloheximide and $\mathrm{PTH}(\mathrm{P}+\mathrm{C})$ received a $30 \mathrm{~m}$ pretreatment with cycloheximide alone. * is indicative of $P<0.05$, as calculated by oneway ANOVA. 


\section{Part 4: Effect of PTH on Npt2a promoter activity}

To confirm that the mechanism whereby PTH decreases Npt2a mRNA is posttranscriptional, luciferase reporter assays were performed to determine whether PTH has any effect on Npt2a gene promoter activity. Luciferase reporter constructs featuring either full-length Npt2a promoter (4700 bp) or a truncated promoter (208 bp) that displays twice the activity as the full-length promoter were transfected into OK cells. These cells were then treated with PTH for 8 or 24 hours, and luciferase activity was calculated relative to Renilla as a transfection control. Reporter assays showed no effect of PTH on Npt2a promoter function with either the full-length promoter or truncated version (Figure 11).

\section{FIGURE 11}

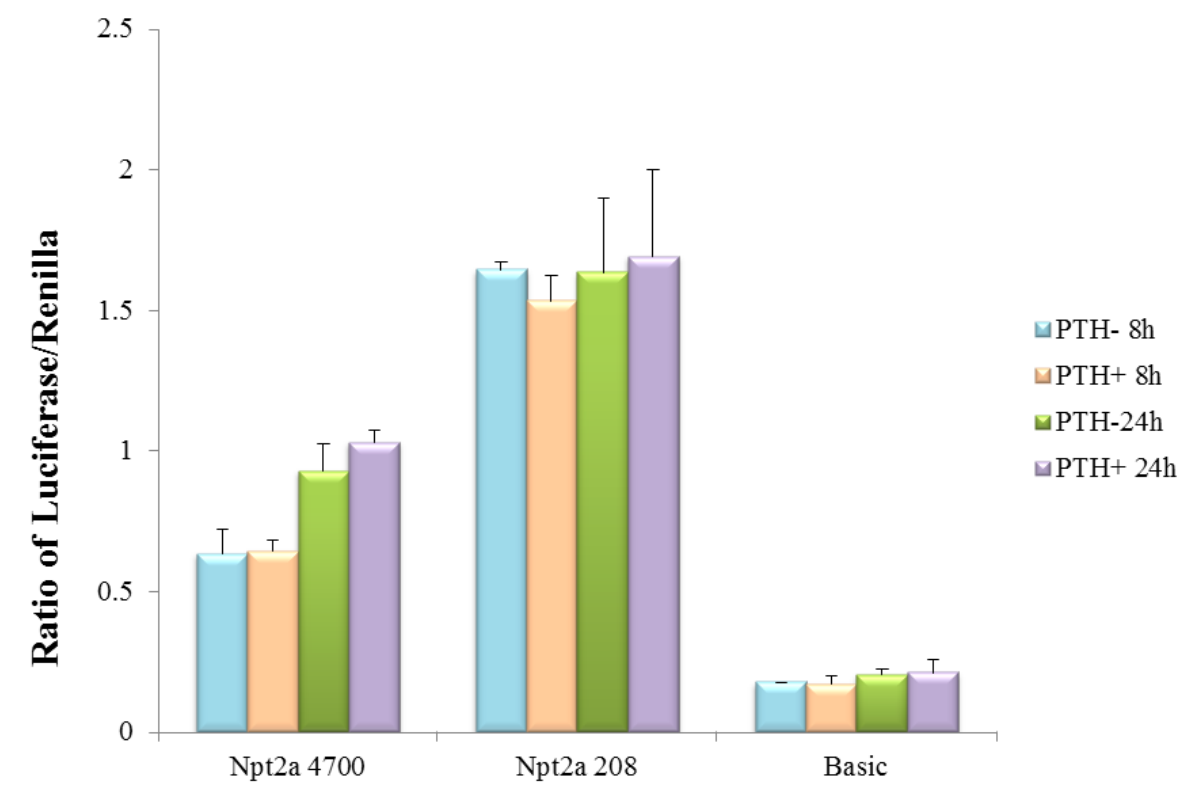

Figure 11: Effect of PTH on Npt2a promoter function. OK cells were co-transfected with Npt2a full-length (4700 bp) or truncated (208 bp) promoter luciferase constructs, and Renilla constructs as internal control, as described in Methods. Luciferase values are shown normalized to Renilla values. 


\section{$\underline{\text { DISCUSSION }}$}

Here we provide evidence that PTH selectively produces a sustained reduction in $\mathrm{Npt} 2 \mathrm{a}$ expression at the protein and mRNA level in the renal proximal tubule. In a mouse model of primary hyperparathyroidism, Npt2a expression remained suppressed while the sodium pump and the PTH receptor displayed no significant changes in expression. PTH stimulation of the proximal tubule induces the rapid endocytosis of Npt2a, followed by lysosomal degradation of the transporter, thus requiring de novo synthesis for the reinsertion of Npt2a back into the membrane. While PTH is a known regulator of Npt2a protein degradation, here we show that PTH also regulates the rates of de novo synthesis through its effects on Npt2a mRNA levels in the cell. In both hyperparathyroid models used in this study, PTH significantly and rapidly reduced Npt2a mRNA expression through post-transcriptional mechanisms. The Npt2a gene promoter has several cis-acting elements in it, including cAMP response elements, introducing the possibility that PTHstimulated cAMP signaling may regulate Npt2a promoter activity. However, we observed no effect of PTH on Npt2a promoter function, either at the $8 \mathrm{~h}$ or $24 \mathrm{~h}$ time point, so while Npt2a gene promoter function is regulated by several factors, PTH is not one of them.

This observation is in accordance with previous studies published by Hilfiker et al. ${ }^{109,116}$. The luciferase reporter assays thus support the notion that the PTH-mediated decrease in Npt2a mRNA is a strictly post-transcriptional process.

Exploring further the mechanism behind the regulation of Npt2a mRNA by PTH, we found that the effect of PTH on Npt2a is dependent on both transcription and translation. We found the basal reduction of Npt2a mRNA to the 50\% level required 8.6 
hours, but PTH greatly reduced that time to just over 2 hours. Inhibiting transcription with actinomycin D while treating cells with PTH completely blocked the ability of PTH to destabilize the transcript. The dependence of PTH-mediated destabilization of Npt2a mRNA on transcription suggests two possibilities - either upregulation of a miRNA or upregulation of a protein is responsible for mediating the PTH response. Inhibiting translation with cycloheximide while treating OK cells with PTH showed that the PTH effect is also dependent on translation. While the rapid reduction of Npt2a mRNA is consistent with miRNA involvement, the observation that this is a cycloheximidesensitive mechanism indicates that enhanced protein synthesis, potentially of an RNAbinding protein, is vital for this destabilization process.

While this is the first report of protein synthesis potentially leading to altered Npt2a mRNA stability in response to PTH, previous studies have demonstrated that Npt2a mRNA stability is regulated in response to various stimuli through differential protein expression. In a study where hypophosphatemia increases Npt2a mRNA levels, Moz et al. ${ }^{3}$ showed that a low phosphate diet in rats induces post-transcriptional stabilization of Npt2a mRNA. In their model of hypophosphatemia, a low phosphate diet induced increased production of renal cytosolic proteins, and that these proteins had a protective effect on the Npt2a mRNA by binding to both the 5' untranslated region (UTR) and 3' UTR. Their work indicates that there is a cis-acting instability element in the protein-binding region of the 3' UTR of Npt2a mRNA ${ }^{3,117}$, and that cytosolic proteins produced in response to hypophosphatemia bind to this region and stabilize the transcript. This influence of serum phosphate levels on Npt2a mRNA stability was also observed in another study of a distal tubule-specific Klotho knockout mouse. This knockout mouse 
displays increased protein expression of Npt2a in the proximal tubule as a result of the Klotho deletion, and yet decreased levels of the Npt2a transcript, perhaps in response to the accompanying hyperphosphatemia ${ }^{56}$. Glucocorticoids, known downregulators of sodium-phosphate cotransport in the proximal tubule, have been shown to reduce both Npt2a protein and mRNA expression. Dexamethasone, a synthetic glucocorticoid, decreases Npt2a mRNA and protein abundance in a manner that is also dependent on both transcription and translation ${ }^{102}$. This study in conjunction with ours suggests the possibility that the characteristics of the specific proteins interacting with the 3 ' UTR of Npt2a mRNA determine the fate of the transcript.

Combined, these studies support the notion that the changes in Npt2a mRNA stability evoked by PTH are due to increased protein synthesis, particularly increased expression of RNA-binding proteins. This mechanism of post-transcriptional regulation of Npt2a by PTH is potentially unique to Npt2a alone, as we observed no effect of chronic PTH on the expression of other PTH targets, including the sodium-potassium pump and NHE3. In the studies described in this chapter, we have demonstrated that PTH induces rapid destabilization of Npt2a mRNA, and that this effect is dependent on transcription and translation. Based on these findings, we propose that PTH upregulates the expression of an RNA binding protein that enhances Npt2a mRNA degradation. Further studies are required to validate this hypothesis. 


\section{CONCLUSION}

Npt2a undergoes downregulation both at the protein level and the mRNA level in response to PTH through distinct mechanisms. PTH induces rapid destabilization of Npt2a mRNA through post-transcriptional mechanisms dependent on the upregulation of an as-yet-unidentified protein. 


\section{CHAPTER V}

\section{SPECIFIC AIM II: PKA AND PKC CONTRIBUTE TO PTH-MEDIATED NPT2A MRNA DESTABILIZATION}

\section{The PTH receptor signaling complex}

In Chapter IV, we established that PTH induces rapid destabilization of Npt2a mRNA in the proximal tubule, and that this destabilization is sustained with chronic PTH. Exploring further the mechanism of PTH-induced Npt2a mRNA destabilization, the goal of the present chapter is to elucidate the signaling events that mediate this response. PTH signaling in the proximal tubule is elicited through its interaction with the PTH receptor type I (PTHR1). The PTH receptor is a member of the G protein-coupled receptor (GPCR) family, with seven transmembrane domains, an extracellular amino terminus, and an intracellular carboxyl terminus. The amino acid sequence of the PTH receptor is conserved across species, with PTHR1 cloned from rat osteoblasts displaying $78 \%$ conservation to the opossum kidney PTHR1, indicating an important systemic role

for the receptor ${ }^{118}$. PTH receptor expression has been identified in a wide array of tissues, including the uterus, gut ovaries, and liver, although expression of the PTHR1 in these tissues is thought to be more important for paracrine/autocrine signaling by the PTH 
related peptide PTHrP, which also couples to the PTHR $1^{119}$. The highest expression of the PTHR1 within the body is found in the bone and kidney. Within the kidney, PTH receptors have been found both in the proximal and distal tubules, as well as the collecting duct ${ }^{120,121}$.

PTH receptors in the proximal tubule are expressed in both the apical and basolateral membranes ${ }^{122}$. The PTH receptor is a member of the class II GPCR family, and possesses the ability to activate several intracellular signaling pathways. It has been shown to couple to several $G$ proteins, including $G_{s}$ and $G_{q}$, leading to the subsequent activation of the cAMP/PKA and PLC/PKC pathways, respectively ${ }^{4}$. These characteristics of ligand-receptor signaling are preserved in cell culture models of the proximal tubule, including opossum kidney cells, where stimulation of the PTHR1 by PTH produces activation of two major signaling pathways: (1) the $\mathrm{G} \alpha_{s} /$ adenylyl cyclase/cAMP/PKA pathway, and (2) the $\mathrm{G} \alpha_{q / 11} / \mathrm{PLC} / \mathrm{IP}_{3} / \mathrm{Ca}^{2+} / \mathrm{PKC}$ pathway ${ }^{123}$.

The signaling pathways activated by PTH are complex and cell-specific, and influence more than just renal phosphate transport. Although the PKA and PKC pathways are the primary pathways activated in the proximal tubule, $\mathrm{PTH}$ receptors throughout the body differ in their structural requirements for signaling pathway activation, particularly when it comes to phospholipase activation. Proximal tubular PTH receptors, for example, are capable of activating different phospholipases from the ones activated by distal tubule PTH receptors ${ }^{120}$. PTH receptors even within the same cell may differ in their G proteincoupling capacities based on their distribution in the apical versus basolateral membrane. Early studies suggested that the PTH receptor in the proximal tubule coupled to distinct G proteins on the apical and basolateral membranes, with apical PTH1Rs thought to 
activate PKC exclusively, whereas basolateral PTH1Rs activated both PKA and PKC ${ }^{124}$. However, Npt2a-GST pull-down experiments by Khundmiri et al. ${ }^{125}$ showed that Npt2a in the BBM of OK cells associates with AKAP79, PKA (both the catalytic and regulatory subunits), and the PTH receptor, and that AKAP-mediated activation of PKA was critical for downregulation of phosphate transport by PTH. While the aforementioned studies provide conflicting evidence for specific $\mathrm{G}$ protein coupling dependent on receptor localization, they nonetheless confirm the role of both $G_{s}$ and $G_{q}$ in mediating PTH signaling within the proximal tubule.

Although the predominant signaling pathways activated by PTH include the cAMP/PKA and PLC/PKC pathways ${ }^{118}$, there are a number of other pathways that PTH has also been shown to activate, and these also appear to be tissue-specific. These include phospholipase A2 in osteoblasts and proximal tubules ${ }^{126}$, phospholipase D in distal tubules $^{120}$, and the mitogen-activated protein kinase (MAPK) pathway in OK cells ${ }^{127}$. In addition to activating PKA, cAMP also activates another category of proteins known as exchange proteins directly activated by cAMP (Epac), which are guanine nucleotide exchange factors for the Ras GTPase family of proteins ${ }^{128}$. Epac1 and Epac2 are both expressed in the brush border of the proximal tubule ${ }^{129}$, and are known regulators of several proximal tubule proteins, including NHE3, aquaporin-2, and the $\mathrm{H}^{+} / \mathrm{K}^{+}$-ATPase. No evidence currently exists for a role of Epac in regulating phosphate transport ${ }^{128}$. PTH signaling decreases $\mathrm{Na}^{+} / \mathrm{K}^{+}$-ATPase activity and expression via extracellular signalregulated kinase (ERK)-mediated activation of PKC- and $\mathrm{PLA}_{2}$-dependent pathways ${ }^{49,71}$. Of the pathways activated by PTH in the proximal tubule, however, PKA and PKC 
remain the most influential in mediating downstream phosphaturic effects of PTH signaling, and in that regard are the most highly studied.

\section{$\underline{\text { PTH-induced PKA signaling }}$}

The PTHR1 is a potent stimulator of cAMP generation and PKA activity through its association with the $\mathrm{G}$ protein $\mathrm{G} \alpha_{\mathrm{s}}$. As shown in Figure 12, when activated by ligandreceptor interaction, $\mathrm{G} \alpha_{\mathrm{s}}$ activates the closely associated adenylyl cyclase (AC), an enzyme that rapidly catalyzes the formation of cyclic AMP (cAMP) from ATP. Within the AC family of proteins, nine isoforms have been identified, and the effects of those isoforms are dependent on "signalosomes" recruited by each specific isoform ${ }^{130}$. As studied in the rat nephron, AC isoforms II, III, VI, VII, and IX are expressed in the proximal tubule, with II, III, and IX confirmed through IHC to be expressed only in the apical membrane (IHC data unavailable for VI and VII) ${ }^{131}$. NHERF-1 has also been found to be important not only in the formation of the apical membrane signalosomes, but also for modulating the amount of cAMP generated in response to PTH stimulation, and its presence is mandatory for cAMP inhibition of $\mathrm{NHE}^{50,132}$.

Within 10 seconds of receptor binding, PTH induces a significant accumulation of $\mathrm{cAMP}^{133}$, which in turn activates protein kinase $\mathrm{A}(\mathrm{PKA})$ by binding to the regulatory subunits, causing dissociation from and activation of the PKA catalytic subunits, thus resulting in the phosphorylation of downstream targets. The cAMP signal is typically terminated through the actions of phosphodiesterases (PDEs) that hydrolyze cAMP to form 5' AMP. In the case of PTH signaling, PTH also rapidly increases PDE activity in a 
dose-dependent and PKA-dependent manner, providing a negative-feedback mechanism for the termination of PTH-induced cAMP signaling ${ }^{134}$.

The dynamics of PTH signaling vary during acute versus sustained signaling. AC is a membrane-bound protein, and the cAMP produced by it is thus produced at the cell membrane. However, this site of cAMP generation is short-lived, as the PTHR1 undergoes endocytosis shortly after stimulation by PTH. This series of events was originally thought to terminate receptor signaling, as association of $\beta$-arrestins with the internalized receptor would desensitize downstream signaling. However, more recent studies have demonstrated that, following endocytosis of the receptor, cAMP generation is sustained. Whereas the previous dogma held that cAMP accumulation occurred only at the cell membrane, studies by Ferrandon et al. ${ }^{133}$ challenge this notion, when they showed that PTH(1-34) was endocytosed to early endosomal compartments, where it not only remained associated with the PTHR1, G $\alpha_{\mathrm{s}}$, and AC, but continued to produce cAMP over a period of 20 minutes. In this manner PTH evokes sustained signal generation in the proximal tubule, even after removal of the original stimulus.

\section{$\underline{\text { PTH-induced PKC signaling }}$}

While the PTHR1 is well known to activate cAMP-dependent pathways by coupling to $G_{s}$, the receptor also couples to $G_{q}$ and is thus a potent stimulator of phospholipase C (PLC)-dependent signaling. Following the activation of PLC by $\mathrm{G}_{\mathrm{q}}$, PLC cleaves membrane phospholipids such as phosphatidylcholine and phosphatidylinositol 4,5-bisphosphate ( $\left.\mathrm{PIP}_{2}\right)$, producing the two signaling molecules inositol triphosphate $\left(\mathrm{IP}_{3}\right)$ and diacylglycerol (DAG). Downstream activation of PKC 
occurs through two processes, as shown in Figure 12. DAG directly binds to and activates $\mathrm{PKC}$, whereas $\mathrm{IP}_{3}$ binds to ligand-gated calcium channels in the membrane of the endoplasmic reticulum. The subsequent rise in intracellular $\left[\mathrm{Ca}^{2+}\right]$ produces activation of PKC in cooperation with DAG. In OK cells, PKC is maximally stimulated 60-90 seconds following activation of the PTHR1 by $\mathrm{PTH}^{32}$. In addition to this PLC-mediated

\section{FIGURE 12}

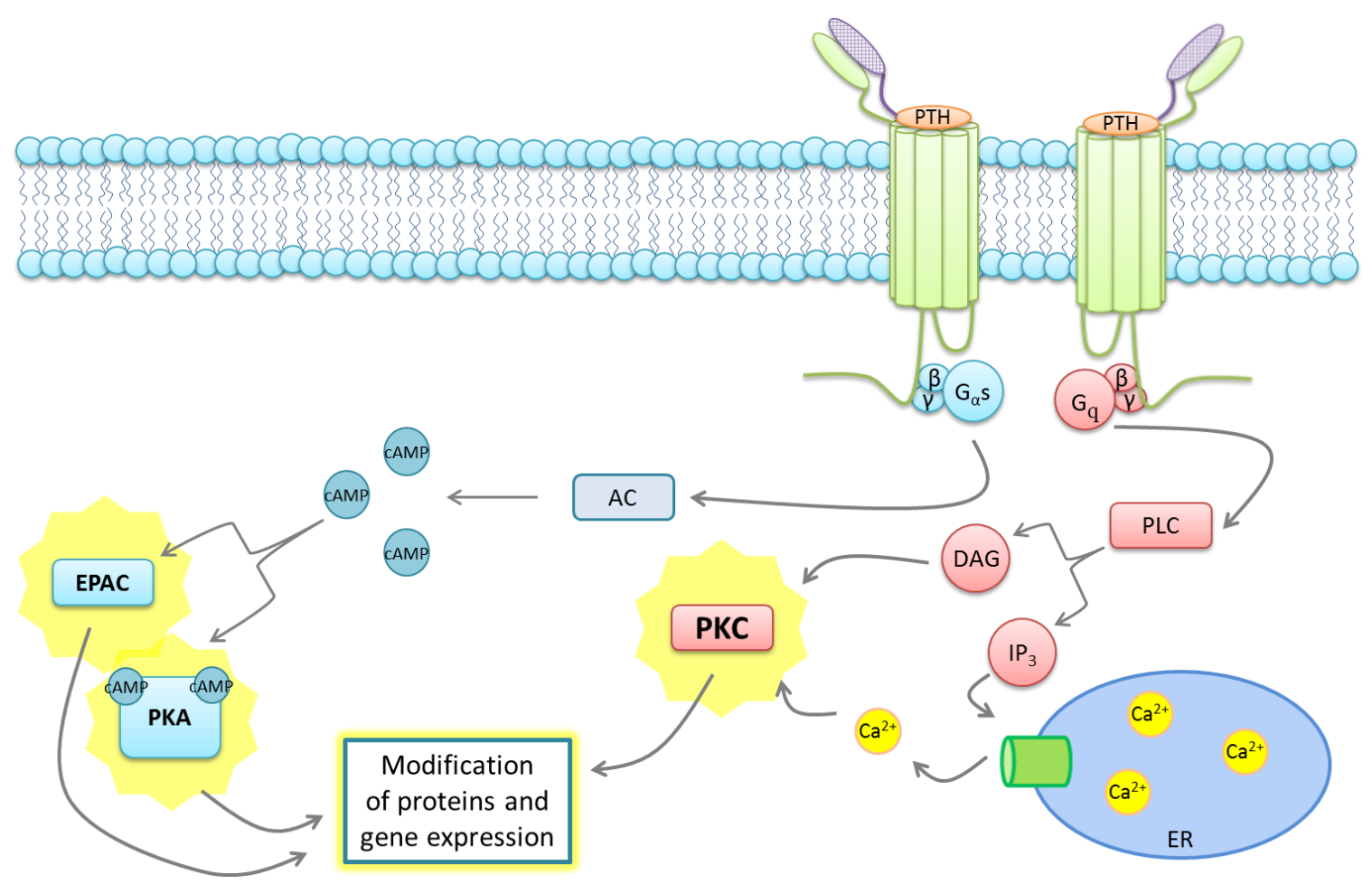

Figure 12: PTH-receptor binding and intracellular signaling in the proximal tubule. Binding of the ligand to the receptor increases the affinity of the intracellular beta-gamma binding region of the $\mathrm{C}$-terminal region of the $\mathrm{PTH}$ receptor for $\mathrm{G}$ proteins, resulting in their subsequent activation and initiation of downstream signaling cascades. $\mathrm{G}_{\alpha} \mathrm{s}$ activates adenylyl cyclase (AC), which increases intracellular [cAMP], resulting in activation of Epac and PKA. $\mathrm{G}_{\mathrm{q}}$ activates phospholipase C (PLC), forming diacylglycerol (DAG) and inositol triphosphate $\left(\mathrm{IP}_{3}\right)$. DAG directly activates $\mathrm{PKC}$, whereas $\mathrm{IP}_{3}$ indirectly activates PKC by releasing $\mathrm{Ca}^{2+}$ from the ER. 
activation of PKC, the PTHR1s expressed in human, mouse, and opossum kidneys are also capable of activating PKC through PLC-independent mechanisms ${ }^{135}$.

PTH-induced signaling is heavily dependent on the interaction of the PTHR1 with accessory proteins. NHERF-1, required not only for apical membrane localization for $\mathrm{Npt} 2 \mathrm{a}$, is also crucial for the regulation of PTH receptor signaling and second messenger generation. In OKH cells, a clonal cell line of the OK WT cell that is deficient in NHERF-1, PTH produces robust levels of cAMP, but fails to stimulate phospholipase C and does not inhibit phosphate transport ${ }^{80}$. In cells that express PTHR1-NHERF-2 complexes, stimulation by PTH potently activates PLC signaling while inhibiting AC through the simultaneous activation of inhibitory $\mathrm{G}_{\mathrm{i} / \mathrm{o}}$ proteins ${ }^{136}$. PTH is thus capable of producing tissue-specific effects in accordance with the specific receptor-protein interactions present within the tissue membrane.

\section{$\underline{\text { PTHR1 endocytosis and desensitization }}$}

The cessation of GPCR signaling is generally dependent on removal of the stimulus and receptor desensitization through endocytosis of the receptor from the plasma membrane. GPCR desensitization is a process that normally requires receptor phosphorylation and/or ubiquitination, promoting the association of $\beta$-arrestins that stop receptor signaling. Resensitization thus usually requires a process of dephosphorylation and removal of $\beta$-arrestin binding. In response to PTH stimulation, the PTHR1 carboxyterminus becomes phosphorylated, promoting $\beta$-arrestin 2 binding and receptor desensitization, followed by endocytosis to an internal compartment ${ }^{137}$. PTH induces a transient polyubiquitination of the PTHR1 by promoting coupled ubiquitination followed 
by deubiquitination through the rapid upregulation of the deubiquitinating enzyme USP $2^{138}$. Following receptor endocytosis and desensitization, GPCRs usually re-sensitize and recycle back to the plasma membrane, which for the PTHR1 occurs within $2 \mathrm{~h}$ after PTH stimulation ${ }^{138}$. The PTHR1 is unique amongst GPCRs in that it does not require dephosphorylation or dissociation from $\beta$-arrestin in order to recycle back to the plasma membrane ${ }^{139}$.

NHERF-1 association with the PTHR1 helps to prolong the expression of the PTHR1 in the plasma membrane and helps sustain PTHR1 signaling. Wang et al. found that NHERF-1 was crucial for preventing $\beta$-arrestin2 from interacting with PTHR1, as well as for maintaining the association of the PTHR1 with $\mathrm{G}_{\alpha} \mathrm{S}^{137}$. The presence of NHERF-1 in the apical and basolateral membranes also protects against receptor desensitization by prolonging the retention of the PTHR1 in the plasma membrane ${ }^{140}$. The Golgi apparatus also appears to play an important role in PTHR1 recycling, as internalized PTHR1 colocalizes with the Golgi, and disrupting the Golgi impairs PTHR1 recycling ${ }^{141}$. In addition to its association with the Golgi, the PTHR1 has also been shown to traffic in and out of the nucleus, although the physiological function of this trafficking pattern is currently unknown ${ }^{142}$.

\section{Pathways involved in Npt2a protein downregulation}

The studies examining the relative contributions of the signaling pathways to the regulation of Npt2a by PTH have been inconclusive at best. Activation of both the PKA and PKC pathways has been shown to downregulate expression of Npt2a at the apical membrane in proximal tubule cells ${ }^{77,143}$. Several studies using cell-based and animal- 
based models have demonstrated that the two signaling pathways both contribute to PTHmediated downregulation of Npt2a protein expression ${ }^{29,80,123,144,145}$, and the relative contribution of each pathway is still under debate.

Several studies support a principal role for PKA signaling over PKC signaling in the sustained downregulation of Npt2a by PTH. In mice that lack adenylyl cyclase isoform 6 (AC6), PTH-induced endocytosis and subsequent lysosomal degradation of Npt2a is absent ${ }^{146}$. Through the use of PTH analogues with selective signaling properties, Nagai et al. showed that the acute down-regulation of phosphate transport in response to PTH involves primarily the cAMP/PKA pathway ${ }^{123}$. Genetic studies in Npt2c KO mice expressing WT or PLC-signaling-deficient PTHR1 showed that the hypophosphatemic response to PTH in PLC-signaling-deficient Npt2c KO mice was only slightly impaired, suggesting that chronic downregulation of Npt2a by PTH is primarily reliant on non-PLC signaling pathways ${ }^{51}$. Support for this conclusion is seen in patients with type Ia or type Ib pseudo-hypoparathyroidism, wherein a mutation in renal proximal tubule $\mathrm{G} \alpha_{\mathrm{s}}$ results in proximal tubule-specific PTH-resistance, resulting in diminished phosphate excretion and hyperphosphatemia ${ }^{147,148}$. This clinical picture implicates a preeminent role for PKA in mediating downregulation of sodium-phosphate cotransport by PTH, as PLC/PKC pathway signaling, which is presumably unaffected by the mutation, cannot compensate for the loss of cAMP/PKA signaling.

Although several studies have implicated PKA as the primary mediator of the PTH phosphaturic response, other studies highlight the importance of PKC signaling over PKA signaling. Whereas Nagai et al. ${ }^{123}$ used PTH analogues to demonstrate a dominant role for PKA signaling, Carpenter et al. ${ }^{149}$ showed preservation of the phosphaturic 
effects of PTH in the absence of cAMP generation, through the use of PTH analogues with $\mathrm{G}_{\mathrm{q}}$-specific-activating properties. In mouse renal proximal tubule cells, inhibition of PKC completely blunted the inhibitory effect of PTH on phosphate transport, while the phosphaturic effect was unaffected by inhibiting PKA $^{58}$. These studies by Cunningham et al. suggest that the inhibitory effect of cAMP on phosphate transport is reliant on downstream PKC activation, which would partially explain the phosphaturic effects of PKA, but does not address the mechanism for PTH-resistant hypophosphatemia in patients with pseudohypoparathyroidism. The differences between these studies may highlight species discrepancies in the contribution of PTH-stimulated signaling pathways to the regulation of phosphate transport by PTH.

PTH also activates several other signaling pathways in proximal tubule, but their roles, both as independent contributors to Npt2a regulation as well as adjunctive to PKA and PKC, in the regulation of Npt2a are not well understood. For instance, FGF23, another major phosphaturic factor, decreases both Npt2a protein and mRNA abundance through MAPK-dependent-signaling in the proximal tubule ${ }^{150}$. Although the MAPK pathway is critical for FGF23-induced hypophosphatemia, which is independent of PKA and PKC activation, the role of MAPK in PTH- and dopamine-mediated inhibition of phosphate transport is unclear ${ }^{58}$. PI3 kinase and phospholipase A2 are additional signaling pathways known to be activated by $\mathrm{PTH}$, but their role in regulating phosphate transport has not been thoroughly investigated. To date, the cAMP/PKA and PLC/PKC pathways are known to be the most influential on phosphate transport. 


\section{Modification of receptor coupling and signaling through PTH fragments}

PTH is a peptide hormone, which in the mature, secreted form is 84 amino acids in length. The first 34 amino acids (1-34) are the biologically active portion of the hormone, responsible for full receptor coupling and subsequent $\mathrm{G}$ protein recruitment and activation (see Figure 12) ${ }^{135}$. Deletion of select amino acids within the peptide hormone results in an incomplete coupling of the ligand to the receptor, and a subsequent incomplete activation of downstream signaling pathways. Thus, modification of the amino acid composition of PTH yields analogues with selective PKA- or PKC-activating properties. Within this chapter, the analogues $\mathrm{PTH}(1-31)$ and $\mathrm{PTH}(3-34)$ are used to preferentially activate the PKA and PKC signaling pathways in order to better define their contribution to PTH regulation of phosphate transport.

PTH(1-31) has been suggested as a relatively cAMP-selective PTHR1 agonist that is deficient for both PLC-dependent and -independent PKC signaling ${ }^{135}$. Treatment of proximal tubular cells with $\mathrm{PTH}(1-34)$ and $\mathrm{PTH}(1-31)$ produces a similar rise in cAMP, indicating that the carboxy-terminal deletion of residues (32-34) does not interfere with Gs activation ${ }^{135}$. PTH analogues truncated at the C-terminus further than the $31^{\text {st }}$ residue, however, fail to stimulate cAMP accumulation ${ }^{120}$. In contrast to $\mathrm{G}_{\mathrm{s}}$ stimulation, the Cterminus (residues 32-34) was found to be critical for $\mathrm{G}_{\mathrm{q}}$ signaling, as demonstrated by Jouishomme et al., who showed that the PTH(29-32) fragment is the smallest hormone fragment capable of stimulating PKC, and that the His32 residue is critical for this stimulation to occur ${ }^{151}$. Loss of the His32 residue thus prevents PTH from activating PKC. This property of His32-receptor coupling prevents PTH(1-31) from activating PKC, while preserving full adenylyl cyclase-stimulating capabilities. 
Truncating PTH at the amino terminus by two amino acids yields the PTH fragment PTH(3-34). Whereas the carboxyl terminus is crucial for activation of PKC, the amino terminus is likewise crucial for the activation of PKA. PTH(3-34), devoid of the amino terminal residues necessary for $\mathrm{G}_{\mathrm{s}}$ activation, selectively activates PKC through non-PLC-dependent mechanisms ${ }^{152}$, albeit less effectively than the full-length analogue, and notably does not activate PKA ${ }^{135}$. Whereas stimulation of the proximal tubule by PTH(1-31) fails to result in an increase of intracellular calcium, stimulation with PTH(334) results in a partial increase, indicating a selective role for $\mathrm{PTH}(3-34)$ in the activation of $\mathrm{PKC}^{120}$. In the experiments outlined in this chapter, human $\mathrm{PTH}(1-31)$ is used as a selective PKA-activating agent, whereas bovine $\mathrm{PTH}(3-34)$ is used as a selective PKCactivating agent.

\section{$\underline{\text { PTH signaling and Npt2a mRNA regulation }}$}

The signaling studies of PTH-mediated downregulation of Npt2a expression have focused solely on the secondary messenger pathways that lead to protein endocytosis and degradation. The PTHR1 has been shown to undergo resensitization and recycling back to the plasma membrane, and the results from Specific Aim I demonstrated that PTHR1 expression remained unchanged with chronic PTH stimulation. While no one has examined signaling events that lead to Npt2a mRNA destabilization, given the dynamics of PTHR1 signaling and the time frame with which those signals cease, we expect there to be some pathway conservation between chronic protein regulation and chronic mRNA regulation by PTH. Based on our results from Specific Aim I, we are proposing that the mechanism of PTH-mediated Npt2a mRNA destabilization involves upregulation of an 
RNA binding protein. Studies in bone examining PTH-responsive induction of gene expression showed that PTH induces rapid upregulation of gene expression through the cAMP-PKA pathway ${ }^{153}$. Given the evidence for downregulation of Npt2a protein expression by PTH, and the sustained cAMP signaling generated by prolonged PTHR1 stimulation, we hypothesize that PKA is the primary mediator of PTH-induced Npt2a mRNA destabilization. We will study PTH-related signaling events and their contribution to PTH-mediated Npt2a mRNA destabilization using the opossum kidney cell line, as previously established in Chapter IV, as a model of PTH-sensitive phosphate transport. We also previously established in Chapter IV a time course of PTH-mediated decrease in Npt2a mRNA that showed a rapid decrease in mRNA levels within $2 \mathrm{~h}$ of PTH, achieving a plateau after approximately $8 \mathrm{~h}$ of PTH stimulation. For the purposes of these experiments, we have defined acute PTH treatment as $30 \mathrm{~m}$ to $2 \mathrm{~h}$, and chronic PTH as $6 \mathrm{~h}$ and $8 \mathrm{~h}$. We have therefore chosen to study the effects on the PKA and PKC signaling pathways for times varying from $30 \mathrm{~m}$ to $8 \mathrm{~h}$. We will use a three-pronged approach to elucidate the signaling mechanisms in PTH-mediated Npt2a mRNA destabilization. First, we will use PTH analogues that are selective for PKA or PKC activation. Second, we will use pharmacologic agents to directly activate either PKA or PKC. Lastly, we will inhibit PKA or PKC activation under conditions of PTH stimulation. 


\section{$\underline{\text { METHODS AND MATERIALS }}$}

Materials: Bovine PTH(1-34), human PTH(1-31), and bovine PTH(3-34) were purchased from Bachem Biosciences Inc. (Kind of Prussia, PA). Phorbol myristate acetate, 8-pCPT2'-O-Me-cAMP, and chlorpromazine were purchased from Sigma-Aldrich (St. Louis, MO). 8-bromo-cAMP, PKC inhibitor peptide, and $\mathrm{H}-89$ were purchased from Calbiochem (La Jolla, CA). Applied Biosystems PCR TaqMan Pre-Developed Assay Reagents and Ambion MirVana miRNA isolation kits and were purchased from Life Technologies (Grand Island, NY). PKA and PKC activity assay kits were purchased from EMD Millipore (Billerica, MA). ATP $\left[{ }^{32} \mathrm{P}\right]$ was purchased from Perkin Elmer (Waltham, MA). Unless otherwise specified, all other chemicals were purchased from SigmaAldrich (St. Louis, MO).

Cell culture: Studies involving time-dependent effects on Npt2a mRNA stability were conducted using the Opossum Kidney $(\mathrm{OK})$ cell line. OK cells were cultured exactly as previously described in Chapter IV. Unless otherwise noted, cells were grown to 90-95\% confluence on 6-well plastic plates (Corning, Tewksbury, MA) and serum-starved overnight prior to treatment.

Protein determination: Protein concentration of OK cell nuclear-free lysates was determined using a bicinchoninic acid (BCA) protein kit (Sigma) using bovine serum albumin (BSA) as a standard. 
Isolate of total RNA: RNA isolation was performed exactly as previously described in Chapter IV.

Quantitative real-time PCR: Reverse transcription and qPCR were performed exactly as previously described in Chapter IV.

Radioactivity assays: All steps were performed on ice unless otherwise indicated. Radioactivity assays for measurement of PKA and PKC activities were performed according to manufacturer's protocol. Briefly, OK cells were treated with PTH analogues and/or PKA or PKC inhibitors as described in Results for times indicated. Cells were washed twice with ice-cold PBS, and lysed in buffer consisting of 5mM Tris- $\mathrm{HCl}$ 50mM mannitol, $\mathrm{pH} 7.4$, and protease and phosphatase inhibitors. Cells were homogenized by 15 passes through a 26 gauge needle. Nuclear-free lysates were prepared by centrifuging lysates at $750 * \mathrm{~g}$ for 5 minutes, and discarding the pellet. $10 \mu \mathrm{L}$ of cell lysate per condition was incubated in a microcentrifuge tube containing $40 \mu \mathrm{L}$ of the reaction reagent mix (prepared according to manufacturer's protocol) and $10 \mu \mathrm{L}$ of the $\left[\gamma_{-}{ }^{32} \mathrm{P}\right] \mathrm{ATP}$ mixture. The kinase reaction took place in a $30^{\circ} \mathrm{C}$ water bath with shaking for 10 minutes. The reaction was stopped by removing $25 \mu \mathrm{L}$ of the reaction mixture and slowly pipetting the aliquot onto phosphocellulose paper. Papers were washed three times with $0.75 \%$ phosphoric acid, followed by one five-minute wash with acetone. Papers were allowed to dry before being added to scintillation vials containing $3 \mathrm{~mL}$ of Econo-safe scintillation cocktail (RPI, Mount Prospect, IL). CPMs were determined using a Tri-Carb 2100TR liquid scintillation counter (Perkin Elmer, Waltham, MA). CPM of negative 
control samples containing no enzyme were used as background control. Specific Radioactivity (S.R.) was calculated by measuring the CPM of $10 \mu \mathrm{L}$ of the $\left[{ }^{32} \mathrm{P}\right] \mathrm{ATP}$ solution, and dividing that value by 5 nmoles ATP to yield units of CPM/nmole ATP. This value was converted to CPM/pmole ATP by dividing the S.R. by 1000 . PKA and PKC activities were calculated by subtracting the background CPM ("B") from each sample (“S”), and calculating as follows:

$\frac{[S-B] \times 2.4}{S . R . \times 10 \mathrm{~min}}=$ pmole phosphate incorporated into substrate peptide/min

This value was further divided by the amount of protein loaded per sample. Each experiment was comprised of two individual samples per condition. Within each experiment, the data was normalized to the average of the controls, and reported as $\%$ change from control.

$\underline{\text { Statistics: }}$ Data are shown as mean \pm SE. The $n$ values represent the number of independent experiments. $P$ values were calculated by Student's $t$-test or by one- or twoway ANOVA, followed by Bonferroni analysis using GraphPad Prism software. A $P$ value $<0.05$ was a priori considered statistically significant. 


\section{$\underline{\text { RESULTS }}$}

\section{Part 1: Effects of PTH analogues on Npt2a mRNA expression}

To examine the relative contribution of the PKA and PKC signaling pathways toward PTH-mediated Npt2a mRNA destabilization, OK WT cells were treated with PTH analogues that relatively selectively activate specific signaling pathways. OK cells were treated with 100nM PTH(1-31) to selectively activate PKA, or $1 \mu \mathrm{M}$ PTH(3-34) to selectively activate PKC, for up to $8 \mathrm{~h}$. The concentration used for PTH(1-31) was

determined according to concentrations published in previous studies ${ }^{135}$, while the optimal concentration for PTH(3-34) was determined by performing dose response curves of stimulated PKA activity and selecting the concentration at which PKA activity showed the least increase. PKA and PKC activity assays were performed to confirm the selective pathway-activating properties of each analogue. As shown in Figure 13C, PTH(1-34) produced a 40\% increase in PKA activity within $15 \mathrm{~m}$ of treatment, which fell to a plateau of about $20 \%$ above baseline by the $2 \mathrm{~h}$ and $6 \mathrm{~h}$ time points. $\mathrm{PTH}(1-31)$ produced a $20 \%$ increase in PKA activity within $15 \mathrm{~m}$ that was sustained after $2 \mathrm{~h}$ of treatment. PTH(3-34) also increased PKA activation by less than $20 \%$ at $15 \mathrm{~m}$, but this effect was transient, decreasing to less than control levels with 6h treatment. Looking at PKC activation by the PTH analogues, PTH(1-34) potently stimulated PKC activity by approximately $50 \%$ at both the $15 \mathrm{~m}$ and $2 \mathrm{~h}$ time points, and increased further still to $200 \%$ by 6 h (Figure 13D). PTH(3-34) produced a modest $20 \%$ increase in PKC activity that was sustained after 6 hours of treatment. PTH(1-31) acute treatment induced a similar increase in PKC activity, but this effect was transient, falling to baseline by $2 \mathrm{~h}$. 


\section{FIGURE 13}

(A)

Effect of PTH(1-31) on

Npt2a mRNA expression

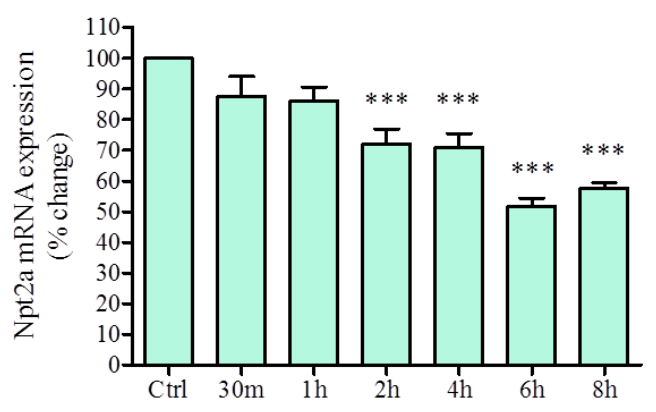

(C)

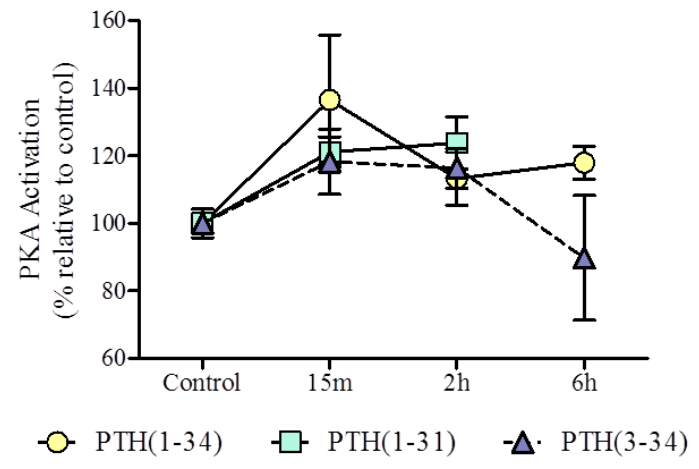

(E)

\section{Contribution of PTH analogues} to Npt2a mRNA degradation

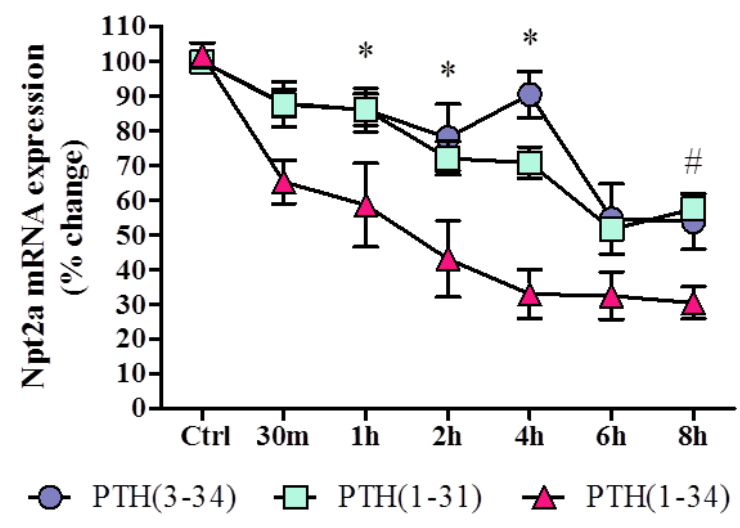

(B)

Effect of PTH(3-34) on

Npt2a mRNA expression

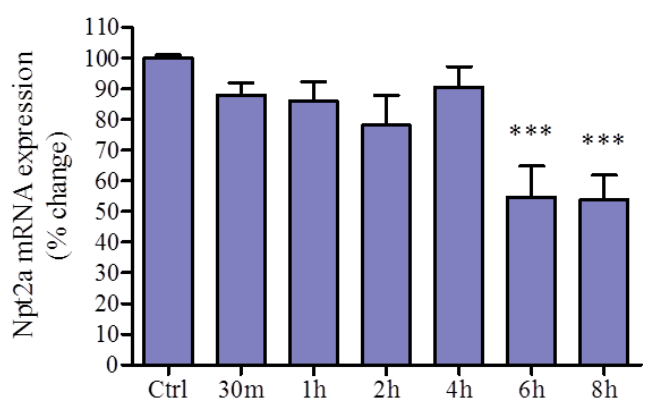

(D)

PKC Activity

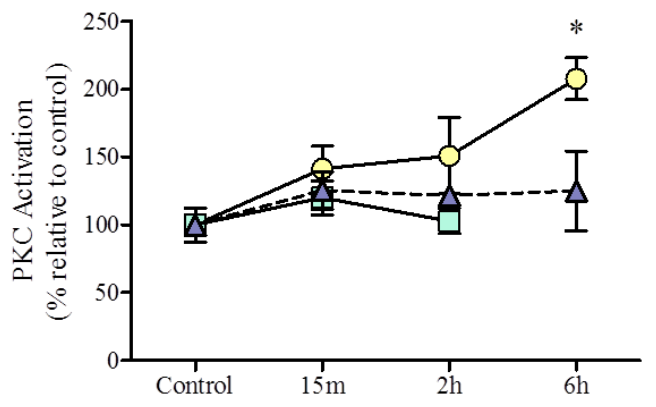

$-0-\operatorname{PTH}(1-34) \quad \square-\operatorname{PTH}(1-31) \quad-\Delta \cdot \operatorname{PTH}(3-34)$
Figure 13: Effect of pathwayspecific PTH analogues on Npt2a mRNA. RT-qPCR with RNA isolated from OK WT cells treated with either (A) 100nM PTH(1-31) $(\mathrm{n}=5)$, or (B) $1 \mu \mathrm{M} \mathrm{PTH}(3-34)(\mathrm{n}=6)$. $* * *$ is indicative of $P<0.001$ versus control. (C) PKA activity and (D) PKC activity stimulated by the PTH analogues $(\mathrm{n}=4) . *$ is indicative of $P$ $<0.05$ as calculated by two-way ANOVA. (E) Comparison of the relative contribution of the PTH

analogues to Npt2a mRNA degradation. * is indicative of $P<0.05$, comparing (1-34) to both (1-31) and (3-34) at times indicated. \# is indicative of $P<0.05$, comparing (1-34) to (1-31) at time point indicated, as calculated by two-way ANOVA. 
Statistical analysis by two-way ANOVA of PKA and PKC activation by the analogues revealed that only $\mathrm{PTH}(1-34)$ produced a statistically significant increase in PKC activity after $6 \mathrm{~h}$ of treatment, and thus further experiments will be necessary to better differentiate between the kinase-activating properties of each analogue. While these analogues were not as specific in activating PKA or PKC as had been previously described, and although both analogues failed to stimulate each kinase as strongly as PTH(1-34), we nonetheless established that $\mathrm{PTH}(1-31)$ preferentially activates PKA whereas $\mathrm{PTH}(3-34)$ preferentially activates PKC.

With the kinase activation properties of the analogues defined, we next determined the effect of the analogues on Npt2a mRNA expression. Over the course of 8 hours, treatment with both $\mathrm{PTH}(1-31)$ and $\mathrm{PTH}(3-34)$ produced a time-dependent decrease in Npt2a mRNA levels, (Figures 13A and 13B), with an approximate 45-50\% reduction from control levels achieved after $8 \mathrm{~h}$ treatment with either analogue. Whereas treatment with PTH(3-34) produced no significant effect on Npt2a mRNA until the 6h time point, $\mathrm{PTH}(1-31)$ significantly decreased Npt2a levels within $2 \mathrm{~h}$ of treatment. As shown in Figure 13E, both PTH(1-31) and PTH(3-34) decreased Npt2a mRNA to a significantly less degree when compared to that induced by PTH(1-34).

\section{Part 2: Effects of direct activation of PKA vs PKC on Npt2a mRNA expression}

To further define the contribution of the PKA and PKC signaling pathways to the PTH-mediated effect on Npt2a mRNA expression, OK WT cells were next treated with direct activators of the respective signaling pathways. To directly activate PKA, OK cells were treated with $10 \mu \mathrm{M}$ 8-bromo-cAMP (8-Br), a cell-permeable cAMP analogue that is 
resistant to degradation by phosphodiesterases. Selective Epac activation was achieved by treating OK cells with 8-CPT-2Me-cAMP $(10 \mu \mathrm{M})$, a cAMP analogue that retains Epac- but not PKA-binding properties ${ }^{154}$. To directly activate PKC, OK cells were treated with 100nM phorbol myristate acetate (PMA), a diacylglycerol mimetic. Over the course of 8 hours, treatment with $8-\mathrm{Br}$ produced a time-dependent decrease in Npt2a mRNA

\section{FIGURE 14}

(A)

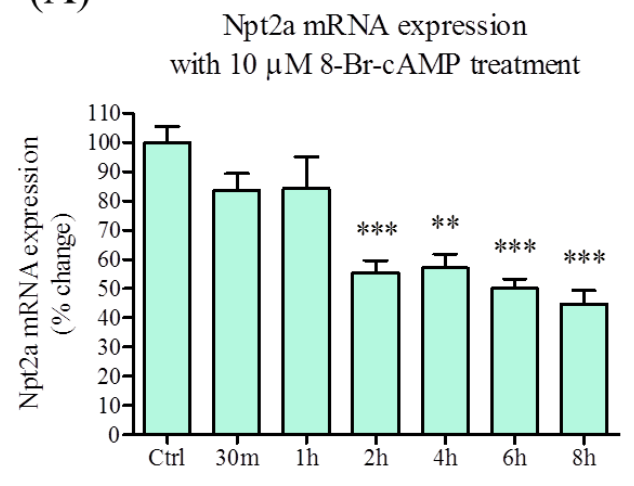

(C)

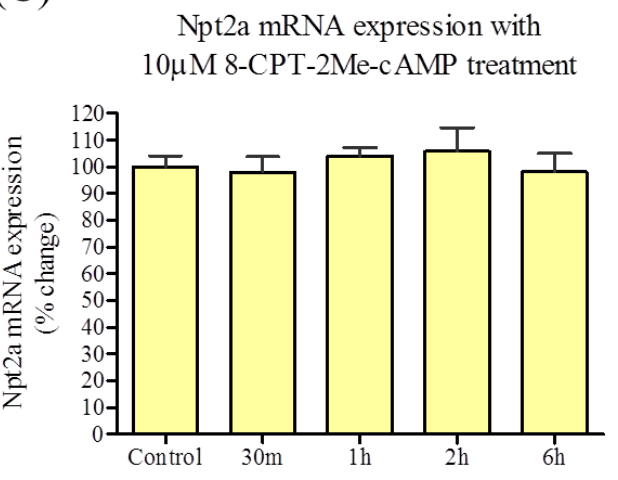

(B)

Npt2a mRNA expression with
100nMPMA treatment

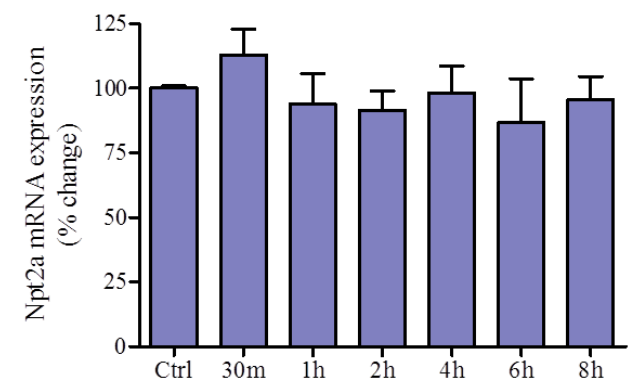

(D)

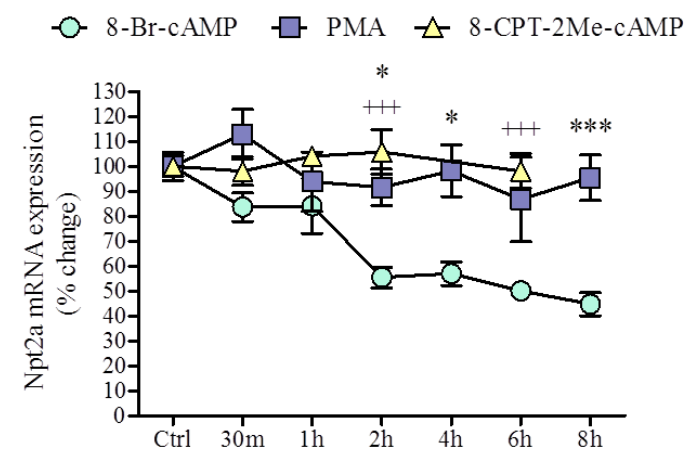

Figure 14: Direct activation of signaling pathways. RT-qPCR was performed with RNA isolated from OK WT cells (as described in Methods) treated with either (A) $10 \mu \mathrm{M}$ 8-Br-cAMP, (B) 100nM PMA, or (C) 10 $\mu$ M 8-CPT-2Me-cAMP, for times indicated. (A) $* *$ and $* * *$ are indicative of $P<0.01$ and 0.001 , respectively, versus control, as determined by one-way ANOVA $(\mathrm{n}=6)$. (D) * and *** are indicative of $P<0.05$ and 0.001 , respectively, for PMA versus $8-\mathrm{Br}$, and +++ is indicative of $P<0.001$ for 8 -CPT versus $8-\mathrm{Br}$, as determined by two-way ANOVA $(\mathrm{n}=6)$. 
levels, comparable to that achieved with PTH alone, with an approximate 55\% reduction from control levels achieved after $8 \mathrm{~h}$ treatment with 8-Br (Figure 14A). Treatment with PMA for up to 8 h, however, failed to produce a statistically significant effect (Figure 14B). Treatment of OK cells with $10 \mu \mathrm{M} 8$ 8-CPT-2Me-cAMP also resulted in no effect on Npt2a mRNA expression (Figure 14C). In comparing the effects of selective PKA, PKC, and Epac activation on Npt2a mRNA expression (Figure 14D), the data strongly suggest a preeminent role for PKA action mediating the effect of PTH on Npt2a mRNA.

$\underline{\text { Part 3: Effects of select pathway inhibition on PTH-mediated Npt2a mRNA }}$ $\underline{\text { destabilization }}$

To support the results of the PTH analogues and direct activation studies, and to assess the ability of PTH to affect Npt2a mRNA levels during the inhibition of the either the PKA or PKC signaling pathways, we next treated OK cells with either an inhibitor of PKA, H-89, or an inhibitor of PKC, PKC inhibitory peptide (PKCi), in the presence and absence of PTH(1-34), over the course of 6h. Cells were pretreated with inhibitor alone for 30m prior to combined PTH-inhibitor treatment for each time point, for a total treatment time of $6.5 \mathrm{~h}$. To confirm the effectiveness of $\mathrm{H}-89$ and $\mathrm{PKCi}$ as inhibitors, we performed assays at $15 \mathrm{~m}$ (with $30 \mathrm{~m}$ pre-treatment) to determine the relative activity of the kinases under the conditions aforementioned. As expected, PTH increased both PKA and PKC activity (Figures 15C and 15D). H-89 alone resulted in a 35\% reduction in basal PKA activity, and completely prevented the activation of PKA by PTH. Inhibiting PKC had no effect on PKA activity, but surprisingly also blunted the ability of PTH to activate PKA. Perhaps even more surprising, H-89 also appeared to inhibit basal PKC activity, 


\section{FIGURE 15}
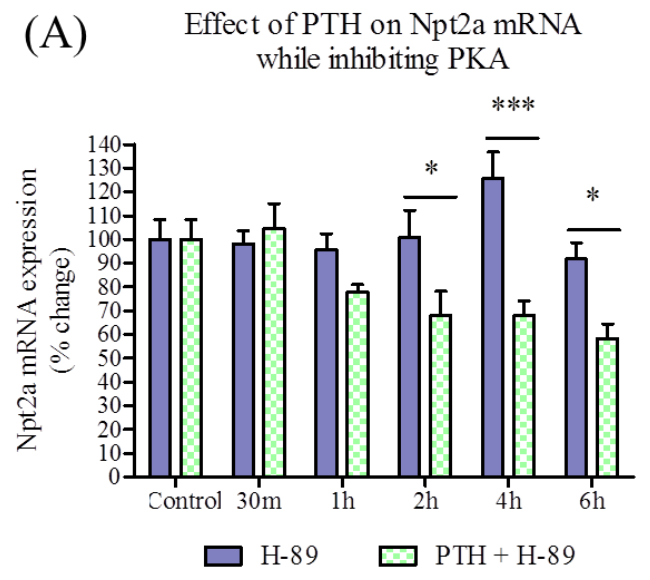

(C)

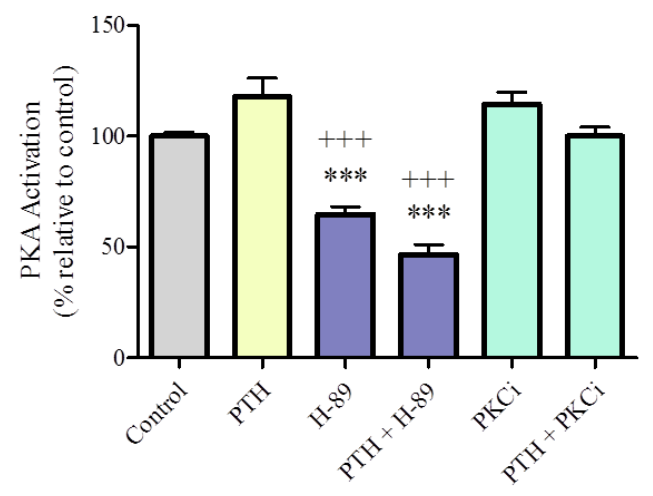

(B)

Effect of PTH on Npt2a mRNA while inhibiting PKC

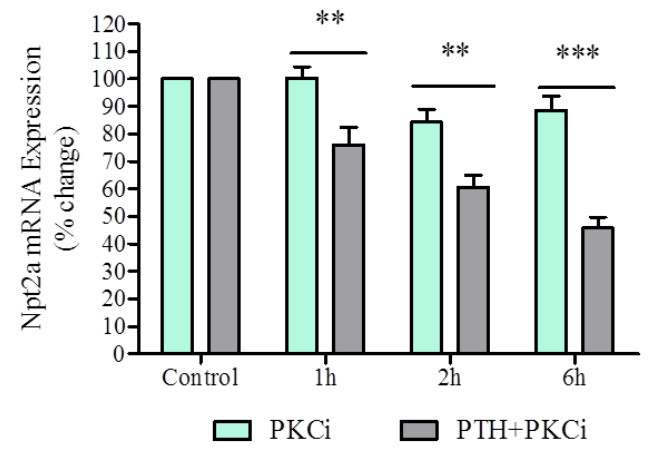

(D)

PKC Activity

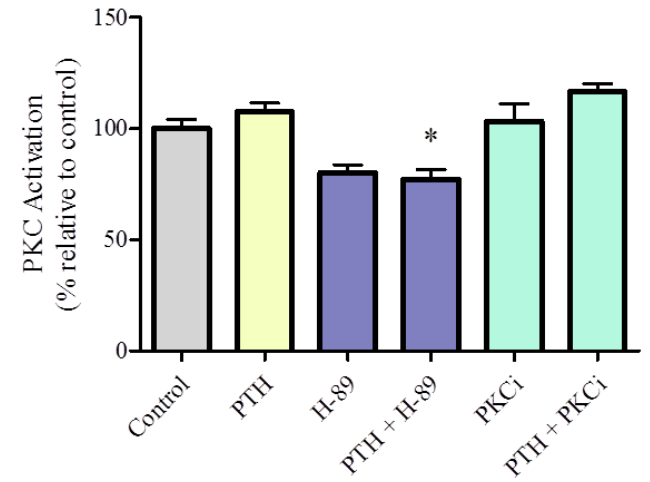

Figure 15: Effect of PTH on Npt2a mRNA during selective pathway inhibition. (A) and (B) RT-qPCR with RNA isolated from OK WT cells was performed as described in Methods. Cells were treated with (A) H-89 alone or combined PTH and H-89, or (B) PKCi alone or combined PTH and PKCi, for times indicated. All conditions (except control) received a $30 \mathrm{~m}$ pre-treatment of (A) $\mathrm{H}-89$ or (B) PKCi alone. *, **, and *** are indicative of $P<0.05,0.01$, and 0.001 , respectively, within time points indicated, as determined by two-way ANOVA (n=4). (C) PKA and (D) PKC activity assays were performed as described in Methods. Treatments shown were performed for $15 \mathrm{~m}$, with a $30 \mathrm{~m}$ inhibitor pretreatment in conditions where inhibitors were present. * and $* * *$ are indicative of $P<0.05$ and 0.001 , respectively, versus control, and +++ is indicative of $P$ $<0.001$ versus PTH, as determined by one-way ANOVA $(n=6)$. 
whereas PKCi failed to inhibit either basal PKC activity or PTH-induced PKC activation. While we were unable to achieve suppression of PKC activation in the presence of the PKC inhibitor peptide following 15m PTH treatment, previous experiments showed that PTH-stimulated PKC activity continues to increase over a $6 \mathrm{~h}$ period. Additionally, while we observed an approximate 15\% increase in PTH-stimulated PKC activity while inhibiting PKC, this is much less than the previously observed $40 \%$ increase in PKC activity following $15 \mathrm{~m}$ PTH(1-34) activity. Further experiments will be necessary to account for the discrepancies observed between the results of the analogue experiments and the kinase inhibition experiments, as well as to further examine the effect of the PKC inhibitor peptide at preventing later increases in PKC activity.

The effect of PTH on Npt2a mRNA expression during selective pathway inhibition was examined next using relatively specific pathway inhibitors. As shown in Figure $15 \mathrm{~A}$, the inhibition of PKA by $1 \mu \mathrm{M} \mathrm{H}-89$ over the course of $6.5 \mathrm{~h}$ had no significant effect on Npt2a mRNA expression. With PKA inhibited, PTH still produced a decrease in Npt2a mRNA, although this effect was delayed compared to the time course of PTH alone, indicating that PKA is important for the early phase of PTH-mediated Npt2a mRNA destabilization. OK cells were next treated with $10 \mu \mathrm{M}$ PKCi, a selective peptide inhibitor of PKC. As with the H-89 experiments, inhibiting PKC alone did not affect Npt2a mRNA expression (Figure 15B). However, the partial inhibition of PKC did not prevent the PTH-mediated decrease in Npt2a mRNA, with significant decreases still observed at the $1 \mathrm{~h}$ time point, and continuing to the $6 \mathrm{~h}$ time point, with an ultimate reduction of 55\% achieved following $6 \mathrm{~h}$ of combined PKCi and PTH treatment, similar to what was achieved with PTH alone. While it appears that the PTH effect on Npt2a 
mRNA was not blunted by the inhibition of PKC, PKC activity assays at the $1 \mathrm{~h}$ and $6 \mathrm{~h}$ time points are required to confirm these results.

\section{Part 4: Effect of inhibition of endocytosis on PTH-mediated Npt2a mRNA destabilization}

Recent studies suggest that chronic PTHR1 signaling occurs with endocytosis of the PTH receptor producing sustained cAMP generation ${ }^{133}$. In addition to their findings that sustained PTHR1 cAMP signaling occurs from early endosomal compartments, Ferrandon et al. also demonstrated that blocking the endocytosis of the ligand-receptor complex diminished the length of cAMP generation from more than 20 minutes down to about five minutes ${ }^{133}$. To test whether this mechanism of PTH signaling influences Npt2a mRNA expression levels, OK cells were treated for 30m with chlorpromazine (CPZ), an inhibitor of clathrin-mediated endocytosis ${ }^{155}$, in the presence and absence of PTH for $2 \mathrm{~h}$. As shown in Figure 16, inhibiting endocytosis did not blunt the effect of PTH on Npt2a mRNA.

\section{FIGURE 16}

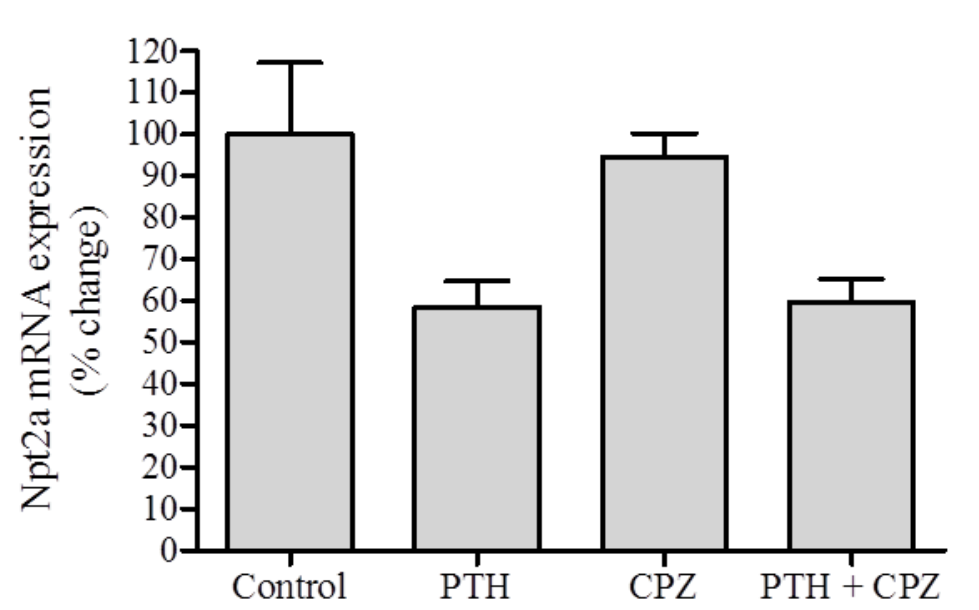

Figure 16: Effect of endocytosis inhibition on PTH-mediated Npt2a mRNA destabilization. RTqPCR with RNA isolated from OK WT cells treated with either 100nM PTH(1-34) for $2 \mathrm{~h}, 40 \mu \mathrm{M}$ chlorpromazine (CPZ) for $2.5 \mathrm{~h}$, or $30 \mathrm{~m}$ chlorpromazine pretreatment followed by combined PTH and chlorpromazine for $2 \mathrm{~h}$. $(\mathrm{n}=2)$ 


\section{$\underline{\text { DISCUSSION }}$}

We examined the relative contribution of PTH-stimulated signaling pathways to the destabilization of Npt2a mRNA using three different approaches: (1) PTH analogues with relatively selective kinase-activating properties, (2) pharmacologic activators of PKA and PKC, and (3) selective pathway inhibition during PTH treatment. The results of these studies suggest that the signaling pathways involved in PTH-induced Npt2a mRNA destabilization are complex and perhaps intertwined. The pharmacologic studies support the notion that PKA signaling is more important for the early phase of the PTHstimulated decreased in Npt2a mRNA, as selective stimulation of PKA using three approaches produced an earlier onset decline in Npt2a mRNA than did selective stimulation of PKC (Figure 17). Use of the PTH analogues study demonstrated that neither the PKA-specific nor the PKC-specific analogue by itself was capable of producing the same degree of Npt2a mRNA destabilization as occurs with activation of both by the full peptide hormone, although each analogue ultimately accomplished a partial reduction. Interpretation of these results in hampered by the fact that neither analogue was either completely specific or as effective in activating the designated pathway as was PTH(1-34). Direct activators of PKA and PKC revealed more clear-cut results, with PKA activation by 8-Br-cAMP producing a pattern of Npt2a mRNA destabilization very similar to that of PTH(1-34), and PKC activation by PMA producing no significant effect. Use of the selective inhibitors of PKA or PKC during PTH stimulation produced results that were also more difficult to interpret, as neither inhibitor was completely specific or robust in inhibiting activation of the desired pathway. 
FIGURE 17

(A) PKA Activation
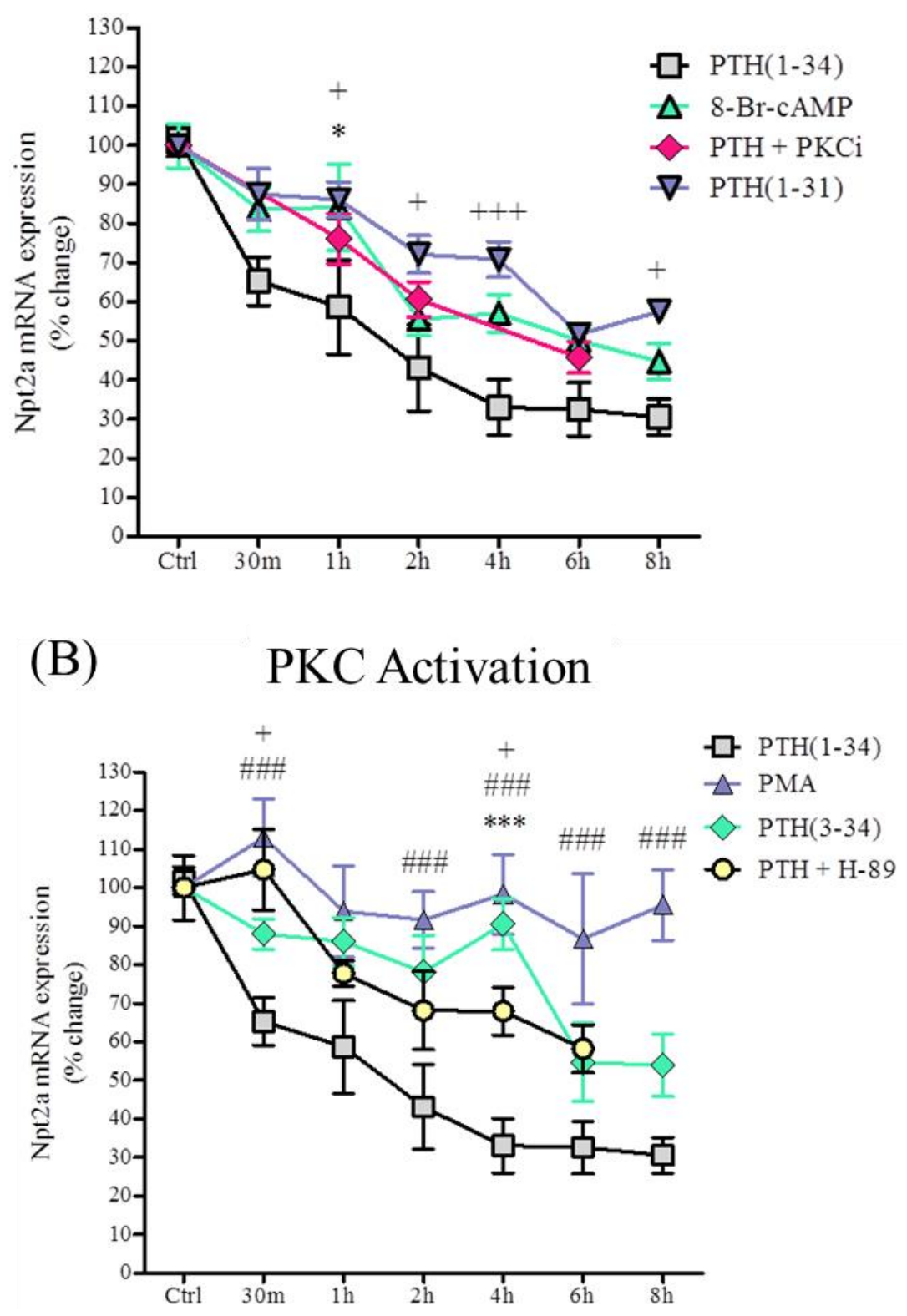

Figure 17: Summary of effects of selective PKA or PKC activation on Npt2a mRNA. (A) Studies in which PKA was activated (treatments with PTH(1-31) [+], 8-Br-cAMP [*], and PTH + PKCi) are shown compared to PTH(1-34) treatment. (B) Studies in which PKC was activated (treatments with PTH(3-34) [*], PMA [\#], and PTH+H-89 [+]) are shown compared to $\mathrm{PTH}(1-34)$ treatment. * and *** (according to respective symbol) are indicative of $P<0.05$ and 0.01 , respectively, as determined by two-way ANOVA, versus PTH(1-34). 
Despite these limitations, the studies do suggest a role for PKA in the early phases of PTH-mediated Npt2a mRNA destabilization, while PKC contributes to the later destabilization of Npt2a mRNA.

The activity assays performed are consistent with the conclusion that the two pathways contribute differentially, depending on whether the PTH stimulation is acute or chronic. Whereas PKA activity increased with $15 \mathrm{~m}$ of PTH(1-34) treatment, this activation decreased by the $2 \mathrm{~h}$ and $6 \mathrm{~h}$ time points, indicating an early role for PKA signaling. PKC activity also increased within the first $15 \mathrm{~m}$ of $\mathrm{PTH}(1-34)$ treatment, but unlike PKA, continued to increase throughout the $2 \mathrm{~h}$ and $6 \mathrm{~h}$ time points. Taking this into account with the fact that the relatively PKC-specific PTH analogue PTH(3-34) failed to produce a decrease in Npt2a mRNA until the $6 \mathrm{~h}$ time point, these results suggest a more prominent role for PKC in long-term PTH signaling. In agreement with this conclusion, Guo et al ${ }^{51}$ recently established that the sustained hypophosphatemic response to PTH in mice is dependent on the PLC signaling pathway. In transgenic mice featuring a PTH receptor incapable of PLC activation, PTH was only able to transiently decrease serum $\mathrm{P}_{\mathrm{i}}$ for two days, after which the hypophosphatemic response decayed. These results support our findings that PKC signaling contributes to the later stages of Npt2a mRNA destabilization, but is not required for early inhibition.

It is interesting to note that $\mathrm{P}_{\mathrm{i}}$-depleted animals fail to produce a hypophosphatemic response to PTH administration, despite normal activation of PKA ${ }^{156}$. The authors of the study concluded that phosphate depletion inhibits cellular metabolic pathways that are independent of cAMP signaling, and that inhibition of those pathways is sufficient to completely blunt the phosphaturic actions of PTH. The signaling pathways 
affected by PTH may have a common end effector, but they also have independent actions that may blunt the influence of the other signaling pathway, and PKA and PKC may thus be able to independently exert downregulatory effects on Npt2a mRNA.

The studies in this chapter point to an early role for PKA and a late role for PKC, but it appears that activation of both are essential for achieving the full decrease in Npt2a mRNA elicited by PTH(1-34). When comparing the effects of the two truncated PTH analogues on Npt2a mRNA versus the original effect of PTH(1-34) (Figure 13E), an interesting dichotomy emerges. In the early phases of PTH signaling, both PTH(1-31) and PTH(3-34) fell significantly short of decreasing Npt2a mRNA levels to the extent that PTH(1-34) did. It is not until the later stages of PTH signaling, hours 6 and 8, that PTH(3-34) and (1-31) appear to catch up with the decreases elicited by (1-34). Part of PTH-induced PKA signaling is produced by sustained cAMP production following endocytosis of the PTHR1 complex. Inhibiting PTHR1 endocytosis is known to prevent the sustained production of $\mathrm{cAMP}$ in response to $\mathrm{PTH}^{133}$. We tested whether this sustained generation of cAMP by PTHR1 endocytosis affected PTH regulation of Npt2a mRNA by treating OK cells with chlorpromazine, an inhibitor of endocytosis, in the absence and presence of PTH. We observed that inhibiting endocytosis failed to prevent PTH-mediated Npt2a mRNA destabilization. Although we are unable to make any conclusive statements regarding the contribution of internalized PTHR1 signaling to the sustained decreased in Npt2a mRNA, these results suggest that the initial generation of cAMP by adenylyl cyclase is sufficient for producing sustained destabilization of Npt2a mRNA. 
These studies suggest the possibility of cross-talk between PKA and PKC, with neither kinase working completely independently of the other. This is not the first time studies have supported this kind of signaling mechanism in the regulation of $\mathrm{Npt} 2 \mathrm{a}$ expression. In studies of Npt2a protein downregulation by $\mathrm{PTH}$, Cunningham et al. proposed a cross-talk-dependent mechanism, wherein PTH stimulates PKA, which in turn activates $\mathrm{PKC}$ in order to cause the ultimate endocytosis of Npt2a from the apical membrane ${ }^{58}$. Dopamine, another phosphaturic agent, also activates both PKA and PKC in the kidney through the $\mathrm{D}_{1}$ receptor, and has also been shown to elicit cross-talk between the two kinases. Yu et al. ${ }^{157}$ demonstrated that dopamine-mediated inhibition of NADPH oxidase involved cross-talk between PKA and PKC $\theta$, with PKA activation preceding and facilitating $\mathrm{PKC}$ activation, and phosphorylated $\mathrm{PKC} \theta$ mediating the resultant inhibition of NADPH oxidase. The activity assays we performed with the PKA and PKC inhibitors support this conclusion, in that $\mathrm{H}-89$ prevented both basal PKA and PKC activity, as well as PTH-induced PKA and PKC activity, and thus PKA most likely plays an additional role in the activation of PKC mediated by PTH.

Although PKA-dependent PKC signaling is one possible explanation for the contribution of the signaling pathways we observed, it does not fully explain the signaling mechanisms involved in PTH regulation of Npt2a mRNA. Whereas Cunningham et al. observed that inhibiting PKC completely abolished the downregulation of Npt2a, we observed preservation of PTH-induced Npt2a mRNA degradation during the inhibition of either pathway. Another possibility aside from kinase cross-talk is that there are multiple ultimate mediators of the mRNA destabilization, and PKA and PKC are each responsible for activating one arm of the multi-pronged cascade. 
This idea was demonstrated by Azarani et al. ${ }^{158}$, who showed that both PKA and PKC could independently decrease NHE3 activity. In this case, Npt2a mRNA destabilization could be accomplished through the activation of several RNA-binding proteins that function as an RNA destabilizing complex, with individual RNA-binding proteins modified by either PKA or PKC. The current studies do not provide distinction between these two possibilities of kinase cross-talk or independent effects, and thus additional studies are required to further define the mechanisms of intracellular signaling leading to destabilization of Npt2a mRNA.

The PKA and PKC pathways are the primary signaling pathways activated by PTH in the proximal tubule, but there are additional pathways also activated by PTH that should be considered. For example, cAMP activates PKA, but it also activates another signaling molecule, Epac. Activated Epac is a demonstrated regulator of some PTHsensitive proximal tubule transporters, including NHE $3^{159}$. To rule out the potential involvement of Epac in Npt2a mRNA regulation, a cAMP analogue that selectively activates Epac was used to treat OK cells. In agreement with previous studies that found that selective Epac activation had no effect on Npt2a protein expression and function ${ }^{58}$, we found no evidence of Epac involvement in the regulation of Npt2a mRNA expression.

The data presented within this chapter provide compelling evidence for the importance of both PKA and PKC signaling in the degradation of Npt2a mRNA, but the lack of total congruity in the results from the three approaches precludes making absolute conclusions on the relative roles of each pathway. Of the three studies performed to examine the relative contribution of the PKA and PKC signaling pathways towards Npt2a mRNA destabilization, two of the studies suggest a role for late PKC involvement, but 
the direct PKC activation study did not support this conclusion. In the direct activation studies, we used PMA to directly activate PKC. We observed no effect of PMA treatment on Npt2a mRNA expression, and these results conflict somewhat with the PTH(3-34) and PKCi studies. However, phorbol esters have been shown to change OK cell morphology by inducing cell rounding, and these changes are not observed with PTH treatment ${ }^{80}$. Thus, while the conflicting PMA data obfuscates the implication for PKC involvement indicated by the other studies, unintended consequences of the use of PMA may be responsible for the lack of an observable effect on Npt2a mRNA. PMA also bypasses activation of PLC, which may activate non-PKC pathways that contribute to Npt2a mRNA destabilization. Additionally, we did not examine intracellular calcium signaling with the use of PMA nor did we measure stimulated PKC activity, and the absence of the initial calcium spike may have prohibited full activation of PKC, thus preventing PKCmediated regulation of Npt2a mRNA.

We are proposing that the upregulated activity and/or expression of an RNAbinding protein is the mechanism behind chronic downregulation of Npt2a by PTH. From the studies herein, we found that the PTH-mediated decrease in Npt2a mRNA is dependent upon both PKA and PKC signaling. Both of these pathways contribute to the end result of mRNA degradation, and either individually or synergistically produce the PTH effect. The dependence of the PTH-induced decrease in Npt2a mRNA on transcription and translation suggests that PKA or PKC signaling is involved in changing the activity and/or expression of transcription factors. Studies by Queiroz-Leite et al. ${ }^{160}$ determined that PTH-mediated PKA and JAK/STAT signaling in OKP cells, a clonal cell line of the OK WT cell, changes the profile of transcription factor expression in the 
nucleus. This change in transcription factor expression may change the expression of RNA-binding proteins that in turn influence Npt2a mRNA stability. Given that we know post-translational modifications produced by PKA and PKC activation mediate Npt2a mRNA destabilization, the next goal in examining the aspects behind regulation of $\mathrm{Npt} 2 \mathrm{a}$ mRNA by PTH was to identify downstream targets that have undergone changes in phosphorylation in response to $\mathrm{PTH}$, and how those targets relate to the control of phosphate reabsorption in the proximal tubule. Further studies addressing this hypothesis of the regulation of RNA-binding proteins in the proximal tubule by PTH and their effects on Npt2a mRNA are outlined in Chapter VI.

\section{$\underline{\text { CONCLUSION }}$}

We conclude that PKA mediates the early phase of PTH-mediated Npt2a mRNA destabilization, whereas chronic down-regulation requires both PKA- and PKC-mediated mechanisms through an intricate, concerted interplay between PKA and PKC signaling, and possibly additional pathways. 


\section{CHAPTER VI}

\section{SPECIFIC AIM III: CHARACTERIZATION OF THE PTH-RESPONSIVE PHOSPHOPROTEOME IN THE PROXIMAL TUBULE}

\section{$\underline{\text { Background }}$}

Activation of the PTH receptor initiates several intracellular signaling cascades, and the actions of kinases and exchange factors produce the collective PTH response. Although much work has been done over the past several decades to elucidate the end results of PTH stimulation in the proximal tubule, the molecular pathways leading to the downregulation of phosphate transport are still poorly understood. We previously established in Chapter IV that PTH destabilizes Npt2a mRNA in a rapid fashion that is dependent on both transcription and translation. In Chapter V, we determined that both PKA and PKC signaling contributes to this effect, with PKA acting early and PKC acting late to promote Npt2a mRNA decay. While PTH-induced signaling pathways may produce upregulated gene expression of specific RBPs, another possibility is that PKA and PKC directly phosphorylate decay-promoting RBPs that have a high constitutive turnover rate. If the latter were true, inhibiting transcription and translation would blunt PTH-mediated destabilization of Npt2a mRNA. Given the results of the two previous aims, we hypothesize that PTH-mediated destabilization of Npt2a mRNA is dependent on 
increased phosphorylation and/or expression of an RNA-binding protein. Presently, the goal of the current chapter is to characterize the PTH-responsive phosphoproteome within the proximal tubule, and characterize PTH-responsive RNA-binding proteins that may mediate Npt2a mRNA decay.

\section{$\underline{\text { Regulation of mRNA expression }}$}

Regulation of mRNA, and in particular mRNA stability, is a dynamic process that involves a host of auxiliary factors, from the cradle to the grave. As soon as transcription begins in the nucleus, the emerging RNA strand is met by proteins that modify the transcript. Capping enzymes add a modified base, 7-methyl guanosine, to the 5' end of the transcript, a spliceosome complex removes introns, and the 3' end undergoes an addition of a polyadenosine (poly(A)) tail that enhances stability ${ }^{161}$. Along with the recruitment of nuclear export proteins, this initial association of proteins with the RNA strand forms a particle termed a ribonuclearprotein (mRNP) complex. The mRNP assembly is then exported to the cytoplasm as an mRNA strand that is comprised of a capped 5' untranslated region (UTR), followed by a coding region, and finally a 3' UTR with a poly(A) tail.

Most mRNA regulatory elements are found within the 5' and 3'UTR regions, where the primary and secondary structures serve as scaffolds for the recruitment of various protein complexes. In the cytoplasm, unique proteins interact with both ends of the mRNA strand. At the 5' end, the protein eIF4E binds the modified guanosine cap ${ }^{161}$. The 3' poly(A) tail is bound by the poly(A) binding protein (PABP). The initiation factor eIF4G forms a link between eIF4E and PABP, thus joining the 5' and 3' ends of the 
transcript and forming a closed-loop conformation. This closed conformation protects the ends of the transcript from exonucleolytic decay and enhances overall transcript stability. The 5'UTR has mostly been implicated in the control of translation, whereas the 3'UTR serves several other critical functions, including nuclear export, cytoplasmic localization, translational efficiency, and mRNA stability ${ }^{162}$.

More than $90 \%$ of human genes undergo alternative splicing, and the highest site for variation produced by alternative splicing is within the 3'UTR, highlighting its importance in producing the highly differential spatial expression profiles of proteins within various tissues throughout the body ${ }^{163}$. Structures within the transcript itself, such as the poly(A) tail or specific nucleotide motifs, are termed cis-acting sequence elements, and these elements dictate the profile of trans-acting factors, such as miRNA and RNAbinding proteins (RBPs), that bind mRNA and determine the fate of the mRNA strand. For instance, sequences within the 3'UTR may dictate the types of location-determining RBPs that regulate mRNA localization ${ }^{162}$, and these distinct localization patterns allow for more rapid and controlled responses to external stimuli. Apart from miRNAs and RBPs, another major category of trans factors that regulate mRNA stability is comprised of small non-coding RNAs (ncRNAs) such as small interfering RNAs (siRNAs) and microRNAs (miRNAs). These small molecules regulate gene expression through partial or complete complementary binding to sequences within mRNA. Near-complementary binding typically prevents translation from occurring, whereas full-complementary binding induces degradation through the recruitment of the Argonaute RNA-induced silencing complex (RISC) ${ }^{161}$. 


\section{Protein-induced mRNA decay}

RNA destabilization leads to the decay of the mRNA strand, which can be initiated either from the ends of the transcript or within the strand via endonucleolytic cleavage $^{161}$, and the rate of RNA decay is modulated by the specific cohort of RBPs interacting with the message. One of the most well characterized family of RBPs that determine mRNA stability bind to a specific cis-element called adenylate-uridylate rich

elements (AREs) ${ }^{161}$. These ARE-binding proteins, or ARE-BPs, typically bind a AUUUA pentamer in a U-rich region anywhere from 40 to 150 nucleotides long ${ }^{164}$. ARE-BPs can bind a spectrum of AREs, however, including ones that lack the canonical AUUUA motif $^{165}$. Signifying its importance as a regulator of gene expression, bioinformatic studies suggest that 5 to $8 \%$ of human genes contain ARE sequences ${ }^{166}$.

HuR is a member of well-known family of proteins that bind AREs and promote message stabilization, either through competitive binding with destabilizing trans factors, or through enhanced interactions of PABP with the poly(A) tail to prevent deadenylation $^{161}$. Other ARE-BPs, including the AU-rich factor 1 (AUF1) and the KH splicing regulatory protein (KHSRP), promote mRNA destabilization ${ }^{161}$. Although AUF1 is most classically known as an RNA destabilizer, new evidence suggests that under certain conditions, AUF1 can also act as a stabilizer or a translational repressor ${ }^{167}$.

\section{The 3' UTR of Npt2a mRNA}

Not much is known about the trans factors that regulate Npt2a mRNA stability. Moz et al. previously demonstrated that hypophosphatemia increases Npt2a expression through stabilization of Npt2a mRNA via increased protein binding to a 71-nucleotide 
cis-acting instability element in the $3^{\prime} \mathrm{UTR}^{117,168}$. However, little work has been published since those studies that detail RBP regulation of Npt2a mRNA in response to physiological stimuli. The predicted secondary structure for the opossum 3' UTR of Npt2a is shown in Figure 18, based on either the minimum free energy of the structure (Figure 18A), or the thermodynamic favorability (Figure 18B). Several RNA-binding proteins are predicted to interact with the 3' UTR of Npt2a mRNA, including ones that modulate stability. As shown in Figure 18C, these RBPs include ELAVL1 (a synonym for HuR), the ARE-BP that confers stability to a transcript, as well as KHSRP, an AREBP that is known to destabilize transcripts. Many of the RBP binding sites also overlap, indicating the possibility of competitive binding for those RBPs.

\section{The PTH-responsive phosphoproteome}

Little is known about the range of downstream targets regulated by PTH signaling in the proximal tubule. The earliest studies of the PTH-responsive phosphoproteome looked at phosphorylation events at the apical membrane that governed the regulation of phosphate transport in response to $\mathrm{PTH}^{169}$. The present studies outlined in this chapter seek to characterize the cell-wide phosphorylation events that occur in response to PTH, and how those changes may relate to the downregulation of Npt2a mRNA. In Chapter IV, we established that PTH induces a rapid decrease in Npt2a mRNA, with an approximate $50 \%$ decrease after $2 \mathrm{~h}$ of PTH stimulation. In Chapter V, we determined that during the early phase, PKA signaling appeared to predominate, while PKC signaling played a more prominent role during the later phases. We have therefore chosen to study the PTHresponsive phosphoproteome at $30 \mathrm{~m}$ and $2 \mathrm{~h}$. While we are proposing that altered activity 
FIGURE 18

(A)

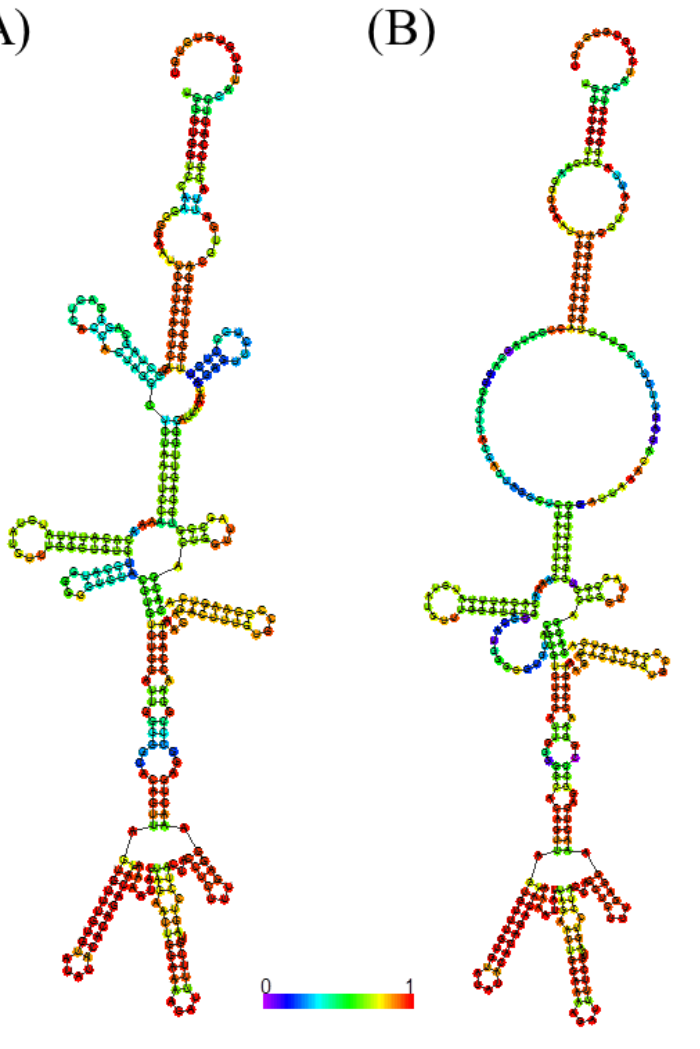

(C)

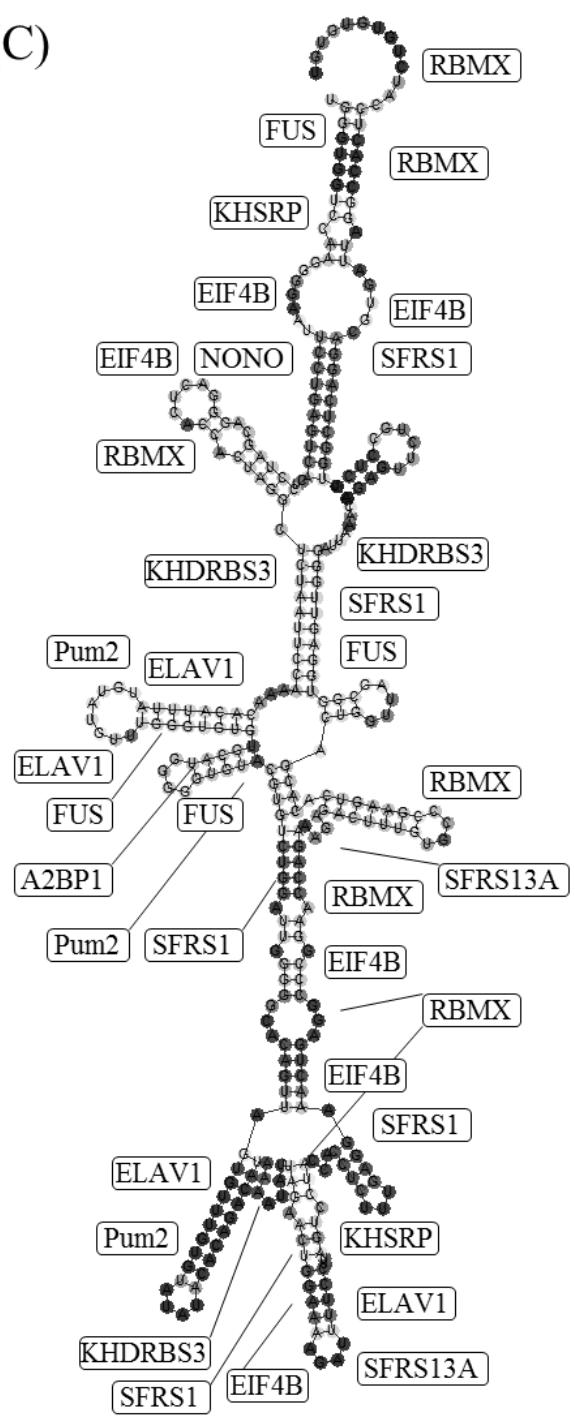

Figure 18: Predicted secondary structure of the 3' UTR of Npt2a mRNA. The sequence for the 3' UTR of opossum Npt2a (obtained from Ensembl.org) was submitted to the Vienna RNA Websuite program for computational analysis ${ }^{170,171}$. (A) Predicted secondary structure based on minimum free energy. (B) Predicted secondary structure based on the thermodynamic favorability of the minimum total base-pair distance to all structures in the ensemble. Color scale indicates likelihood of base-pair interaction or, for unpaired bases, likelihood of being unpaired, with blue representing unlikely and red representing highly likely. (C) Map of likely RBP interactions with the 3' UTR of Npt2a mRNA, as predicted by the RBPDB (RNA-Binding Protein Database) server ${ }^{172}$. 
and/or expression of an RBP is the mechanism behind PTH-mediated destabilization of Npt2a mRNA, we have not yet identified any potential effectors of the decay response. Characterizing the PTH-responsive phosphoproteome will not only provide candidate RNA-destabilizing targets for further exploration of the aspects behind Npt2a mRNA destabilization, but it will also provide novel hypothesis-generating data for research projects to come. 


\section{$\underline{\text { METHODS AND MATERIALS }}$}

Materials: Bovine PTH (1-34) was purchased from Bachem Biosciences Inc. (King of Prussia, PA). Magnetic RNA-binding protein immunoprecipitation (Magna RIP) kits were obtained from EMD Millipore (Billerica, MA). Antibodies against KHSRP and Roquin-2 were purchased from Novus Biologicals (Littleton, CO). All other reagents were obtained from Sigma (St. Louis, MO) unless otherwise indicated.

PTH-D1 mouse model: Kidneys from PTH-D1 and age-matched control mice, as described in Chapter IV Methods, were used for IHC analysis of KHSRP and Roquin-2 expression.

Cell culture: Studies involving differential RBP expression in response to PTH were conducted using the Opossum Kidney (OK) cell line. OK cells were cultured exactly as previously described in Chapter IV. Unless otherwise noted, cells were grown to 90-95\% confluence on 6-well plastic plates (Corning, Tewksbury, MA) and serum-starved overnight prior to treatment.

Protein determination: Protein concentration of OK cell nuclear-free lysates was determined using a bicinchoninic acid (BCA) protein kit (Sigma) using bovine serum albumin (BSA) as a standard. 
Liquid chromatography-mass spectrometry: OK WT cells were grown to confluence in T-75 flasks, serum starved overnight, and treated with either 100nM PTH(1-34) for $2 \mathrm{~h}$ or MEM (as control). Following treatment, cells were washed twice with ice-cold PBS and lysed in a $5 \mathrm{mM}$ Tris $50 \mathrm{mM}$ mannitol buffer, $\mathrm{pH} 7.4$, containing protease and phosphatase inhibitor cocktails. Cells were homogenized by 15 passes through a $26 \mathrm{G}$ needle, and nuclei were discarded by centrifuging the samples at $750 * \mathrm{~g}$ for $5 \mathrm{~min}$ and retaining the supernatant for mass spectrometry analysis. Phosphoproteomic analysis of control and PTH samples was performed with the assistance of Dr. Michael L. Merchant ${ }^{173}$, University of Louisville, who enriched for phosphopeptides using sequential enrichment with $\mathrm{TiO}_{2}$ and IMAC resin, and identified proteins using high sensitivity, high mass accuracy LCMS. Resulting proteins were identified according to their UniProt ID symbol $^{174}$.

Gene identification and pathway analysis: UniProt ID symbols were individually converted to gene names through Uniprot.org ${ }^{174}$. The functions of specific RNA-binding proteins identified within the phosphoproteome were also determined through Uniprot.org. Once gene names for all 1182 proteins were obtained, the gene list was submitted to DAVID for gene ontology analysis ${ }^{175,176}$. Opossum genes not recognized by DAVID software were converted to the orthologous human gene name (determined through Ensembl.org ${ }^{177}$ ) prior to DAVID analysis. Gene ontology for the dataset was analyzed against the dataset for the human genome. 
Computational analysis of RNA-protein interaction prediction: The likelihood of RNAprotein interaction was predicted using the RNA-Protein Interaction Prediction (RPISeq) software from the Dobbs and Honavar laboratories (http://pridb.gdcb.iastate.edu/RPISeq/) ${ }^{178,179}$, with opossum Npt2a 3' UTR, KHSRP protein, and Roquin-2 protein sequences obtained from Ensembl.org. Random Forest (RF) or Support Vector Machine (SVM) classifiers were used to evaluate likelihood of interaction, with 0.5 and above considered the cutoff value for a positive prediction probability of RNA-protein interaction.

Isolation of crude membrane, crude nucleus, and cytosol: Cells were treated as described, washed once with ice-cold PBS, and lysed in lysis buffer consisting of $5 \mathrm{mM}$ Tris $50 \mathrm{mM}$ Mannitol. Cells were lysed by passing the suspension through a 26G needle 15 times. Homogenate was spun at $1500 * \mathrm{~g}$ for $5 \mathrm{~min}$, and pellet was collected for crude nuclear fraction. Supernatant was transferred to a fresh tube and spun at $30,000 * \mathrm{~g}$ for $45 \mathrm{~min}$ to pellet crude membranes (including the plasma membrane and endoplasmic reticulum), and the supernatant of spin two was collected as the cytosolic fraction.

$\underline{\text { Sucrose density gradient centrifugation: Cells were grown to confluence in T-75 flasks }}$ and treated with low phosphate media or regular phosphate media for $24 \mathrm{~h}$. Cells were rinsed twice with ice-cold PBS and scraped in a buffer containing 5mM Tris- $\mathrm{HCl}, 50 \mathrm{mM}$ mannitol, and phosphatase and protease inhibitors. Cells were homogenized with 10 passes through a 26G needle, spun at $1500 * \mathrm{~g}$ for $5 \mathrm{~min}$, and pellet discarded. $1 \mathrm{mg}$ of protein was loaded on top of a sucrose gradient made of stacked $200 \mu \mathrm{L}$ layers of 5 to 
$40 \%$ sucrose in Tris-Mannitol buffer. Gradients were centrifuged using a SW55 rotor at $32,400 \mathrm{rpm}$ for $16 \mathrm{~h}$. One $150 \mu \mathrm{L}$ fraction was first collected from the top, followed by $200 \mu \mathrm{L}$ fractions for the rest of the gradient. Specific cell compartment fractions were determined by blotting each fraction for markers of the endoplasmic reticulum, plasma membrane, cytosol, and nucleus. Once the location of compartments was identified, fractions corresponding to a cell compartment were pooled, and $100 \mu \mathrm{L}$ of each pooled fraction was combined with $33 \mu \mathrm{L}$ Laemmli buffer and heated at $95^{\circ} \mathrm{C}$ for $5 \min .125 \mu \mathrm{L}$ of each sample were loaded per lane onto a $10 \%$ polyacrylamide gel.

Western blot analysis: Following protein estimation, equal amounts of membrane, nuclear, or cytosolic proteins were heated in Laemmli buffer at $95^{\circ} \mathrm{C}$ for 5 minutes to denature proteins. Protein samples were separated by SDS-PAGE and analyzed by Western blot, as previously described in Chapter IV.

RNA-binding protein immunoprecipitation (RIP): RIP experiments were performed according to manufacturer's protocol. Briefly, OK WT cells were grown to $95 \%$ confluence in T-75 flasks (Corning, Tewksbury, MA), and serum-starved overnight prior to treatment. Flasks were treated with 100nM PTH(1-34) or MEM (as control) for $2 \mathrm{~h}$. Afterwards, cells were rinsed twice with ice-cold PBS and scraped into $10 \mathrm{~mL}$ of PBS. Cells were collected by spinning at $1500 \mathrm{rpm}$ for $5 \mathrm{~min}$ at $4{ }^{\circ} \mathrm{C}$, and the supernatant was discarded. Cell pellets were resuspended in an equal pellet volume of RIP lysis buffer containing protease and RNase inhibitors. Lysates were incubated on ice for $5 \mathrm{~min}$ and then stored overnight at $-80^{\circ} \mathrm{C}$. The following day, magnetic beads were prepared for 
immunoprecipitation by incubating the beads with $5 \mu \mathrm{g}$ antibody of interest or isotype IgG for $1.5 \mathrm{~h}$ at room temperature, then washed three times. RIP lysates collected previously were quickly thawed and centrifuged at $14,000 \mathrm{rpm}$ for $10 \mathrm{~min}$ at $4^{\circ} \mathrm{C} .100 \mu \mathrm{L}$ of the supernatant was transferred to each antibody-beads complex. $10 \mu \mathrm{L}$ of the supernatant was saved at $-80^{\circ} \mathrm{C}$ as input for comparison of RT-PCR results. All of the tubes containing the antibody-beads complexes were incubated with rotation overnight at $4^{\circ} \mathrm{C}$. The next day, beads were washed 6 times with cold RIP wash buffer. Following the final wash, each immunoprecipitate was resuspended in $150 \mu \mathrm{L}$ of Proteinase K Buffer consisting of $117 \mu \mathrm{L}$ RIP wash buffer, $15 \mu \mathrm{L} 10 \%$ SDS, and $18 \mu \mathrm{L}$ proteinase $\mathrm{K}$. All tubes were incubated at $55^{\circ} \mathrm{C}$ for $30 \mathrm{~min}$ with shaking to digest the protein. Supernatants were collected and transferred to a new tube, and processed for RNA isolation.

Isolation of RNA: RNA from digested RIP samples was isolated exactly as previously described in Chapter IV, from the addition of phenol:chloroform:isoamyl alcohol onward.

Reverse transcription and real-time PCR: RT-qPCR was performed on RNA isolated from RIP experiments exactly as previously described in Chapter IV. PCR reactions were performed in triplicate, and the three data points averaged to obtain one $\mathrm{Ct}$ value per sample. IP efficiency was determined by comparing the fold difference (calculated as described in Chapter IV Methods) of IP conditions to IgG controls performed for each condition. 
Immunohistochemistry: IHC was performed as described in Chapter IV. Antibodies against KHSRP and Roquin-2 were used at a dilution of 1:1000. Secondary biotinconjugated antibodies were also used at a dilution of 1:1000.

Statistics: Data are shown as mean \pm SE. The $n$ values represent the number of independent experiments. $P$ values were calculated by Student's $t$-test or by one- or twoway ANOVA, followed by Bonferroni analysis using GraphPad Prism software. A $P$ value $<0.05$ was a priori considered statistically significant. 


\section{$\underline{\text { RESULTS }}$}

\section{Part 1: The PTH-responsive phosphoproteome}

Through proteomic analysis, we identified 1183 individual proteins whose phosphorylation significantly changed in response to PTH. The gene names of 1139 phospho-proteins were recognized by DAVID for functional analysis. DAVID mapped 852 proteins out of the 1139 submitted to one or more biological process categories. DAVID analysis identified 245 categories of biological processes regulated by PTH. As shown in Figure 19A, processes involved in transcription and its regulation were the top biological processes altered by PTH, followed by intracellular signaling and phosphate metabolism. 204 unique proteins, or $17.2 \%$ of the PTH-responsive phosphoproteome, were identified as effectors of transcription or regulators of transcription. Perhaps not surprisingly, protein localization (77 proteins) and amino acid phosphorylation (70 proteins) are also major biological processes regulated by PTH. Processes governing cell cycle and macromolecular metabolism rounded out the top ten biological processes modified by PTH.

DAVID also mapped 723 proteins to 96 categories of cellular location (Figure 19B). The cellular compartment identified as having the most proteins (253) regulated by PTH is comprised of non-membrane-bounded organelles, which is defined as a structure of distinct morphology and function, but not bound by lipid bilayer. Types of structures included in this category are ribosomes, the cytoskeleton, and chromosomes. Other major cellular components regulated by PTH include the cytoskeleton (160 proteins), intracellular organelle lumens (148 proteins), and the nuclear lumen (136 proteins). 


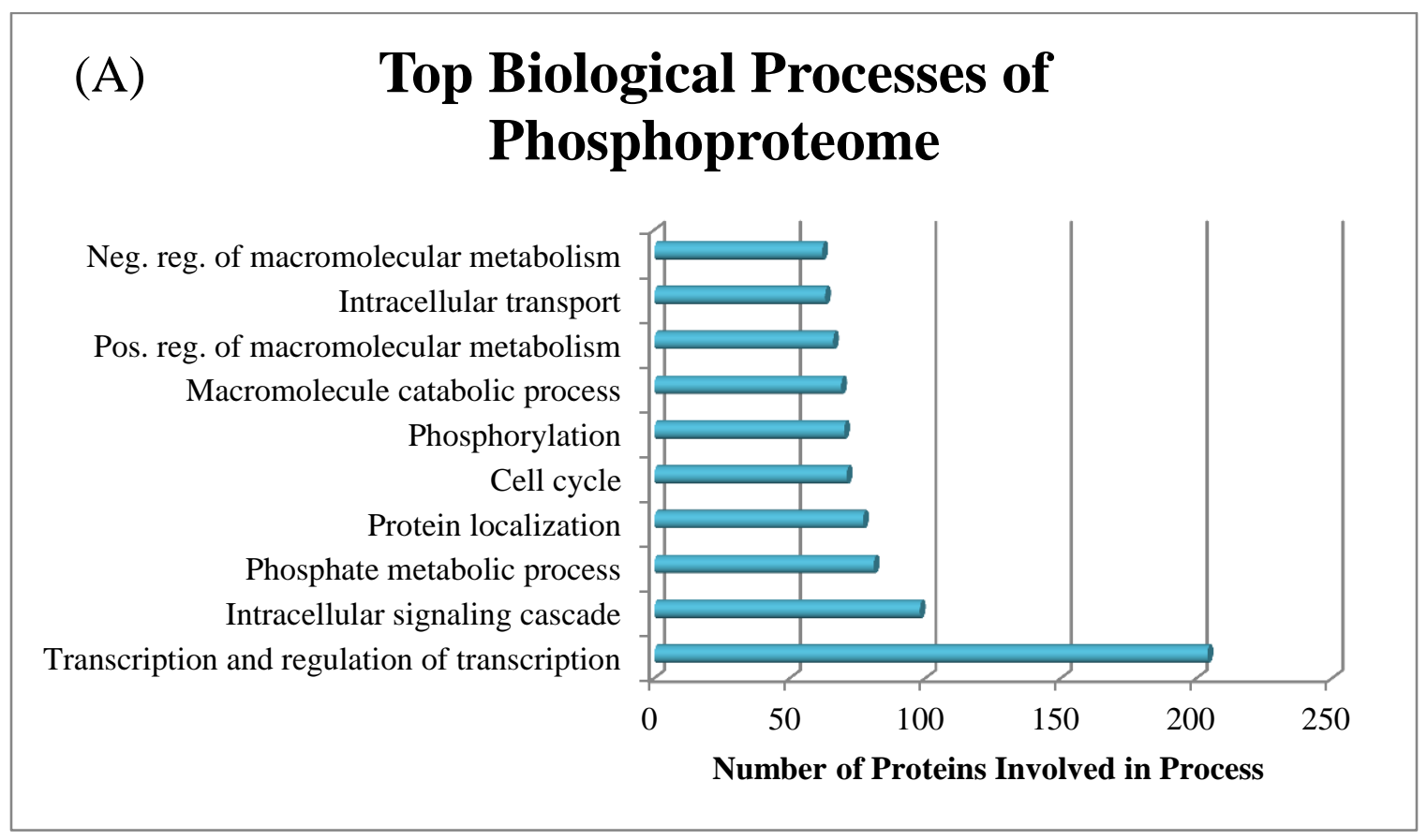

(B)

\section{Top Cellular Components of Phosphoproteome}

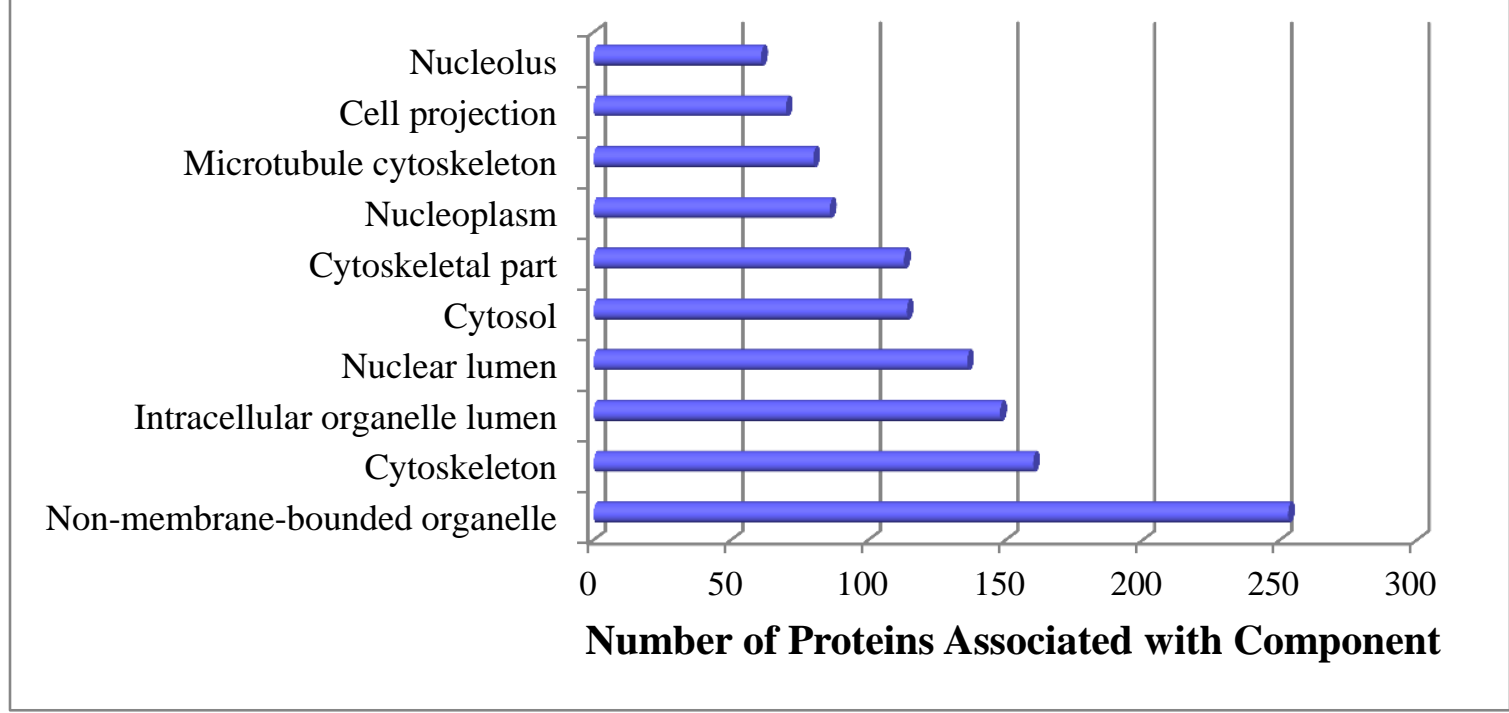

Figure 19: PTH-responsive phosphoproteome. Proteins identified as having undergone significant changes in their phosphorylation status in response to $\mathrm{PTH}$ were subjected to DAVID analysis. (A) Top 10 biological processes of the phosphoproteome. (B) Top cellular components of the phosphoproteome. 
Of the 1139 phospho-proteins identified by DAVID gene ontology analysis of molecular functions, 68 proteins were categorized as having RNA-binding properties. 30 of the phospho-RBPs underwent significant increases in their phosphorylation status within 30m of PTH treatment (Table 1, pages 101-102). 16 of the RBPs only exhibited increased phosphorylation after $2 \mathrm{~h}$ of PTH treatment (Table 2, page 103), and the other 22 RBPs underwent significant decreases in their phosphorylation status in response to PTH (Table 3, page 104). Within the category of fast-responding RBPs, PRPF31, a ubiquitously expressed pre-mRNA splicing factor ${ }^{180}$, underwent the largest increase in phosphorylation status. SRRM2, another splicing factor ${ }^{174}$, also had increased phosphorylation after $30 \mathrm{~m} \mathrm{PTH}$, and was the most increased of all the RBPs at the $2 \mathrm{~h}$ time point.

Whereas most RBPs experienced increased phosphorylation within 30m of PTH treatment, some only responded after $2 \mathrm{~h}$ of PTH stimulation. Of the delayed-responding RBPs, RPL7, a regulatory protein of the translation apparatus, experienced the biggest increase in phosphorylation. Also prominent in the delayed-responding RBP category was the cap-recognition protein eIF4A1, which also experienced a significant increase in phosphorylation after $2 \mathrm{~h}$ of PTH treatment.

The third category of RBPs consists of 22 RBPs that underwent a decrease in phosphorylation in response to PTH (negative-responding RBPs). Several underwent a transient dephosphorylation in response to $30 \mathrm{~m} \mathrm{PTH}$, but regained their basal phosphorylation after $2 \mathrm{~h}$. Included in that category are ZC3HAV1, PDCD11, and SCAF1, proteins involved in viral mRNA degradation, ribosomal RNA biogenesis, and pre-mRNA splicing, respectively. NANOS3 experienced a transient dephosphorylation 
with 30m PTH, but after $2 \mathrm{~h}$ had phosphorylation levels 33 times greater than basal phosphorylation. EIF4G3, or eukaryotic translation initiation factor gamma 3, is a component of the protein complex that recruits ribosomes to mRNA, and in response to PTH, underwent a transient decrease in phosphorylation that increased above baseline following $2 \mathrm{~h}$ PTH treatment.

RNA-binding proteins serve several different functions, and only a subset of RBPS influence RNA stability. Preliminary functional analysis of the phospho-RBPs identified two RBPs that are known to modulate mRNA stability. The first, RC3H2, responded early to PTH treatment, with a significant increase in phosphorylation within $30 \mathrm{~m}$ of PTH that was sustained after $2 \mathrm{~h}$. $\mathrm{RC} 3 \mathrm{H} 2$, more commonly referred to as Roquin2 , is an RBP that promotes constitutive mRNA decay ${ }^{181}$. RPISeq software ${ }^{178,179}$ predicted through the use of Random Forest (RF) or Support Vector Machine (SVM) classifiers that Roquin-2 potentially interacts with the 3'UTR of Npt2a mRNA, with an SVM classifier value of 0.93 . The second phospho-RBP of interest, KHSRP, only experienced an increase in phosphorylation after $2 \mathrm{~h}$ of PTH. KHSRP is a multi-functional RBP, and one of its primary functions is to regulate mRNA stability ${ }^{182,183}$. RPISeq also predicted that KHSRP can interact with the 3'UTR of Npt2a mRNA, with an RF classifier of 0.85 and an SVM classifier of 0.73. Predictive values above 0.5 are considered a positive indicator of protein-RNA interaction. As an initial analysis of the PTH-responsive RBPs, we chose to further explore the roles of KHSRP and Roquin-2 in the PTH response and their potential interaction with $\mathrm{Npt} 2 \mathrm{a}$ mRNA. 


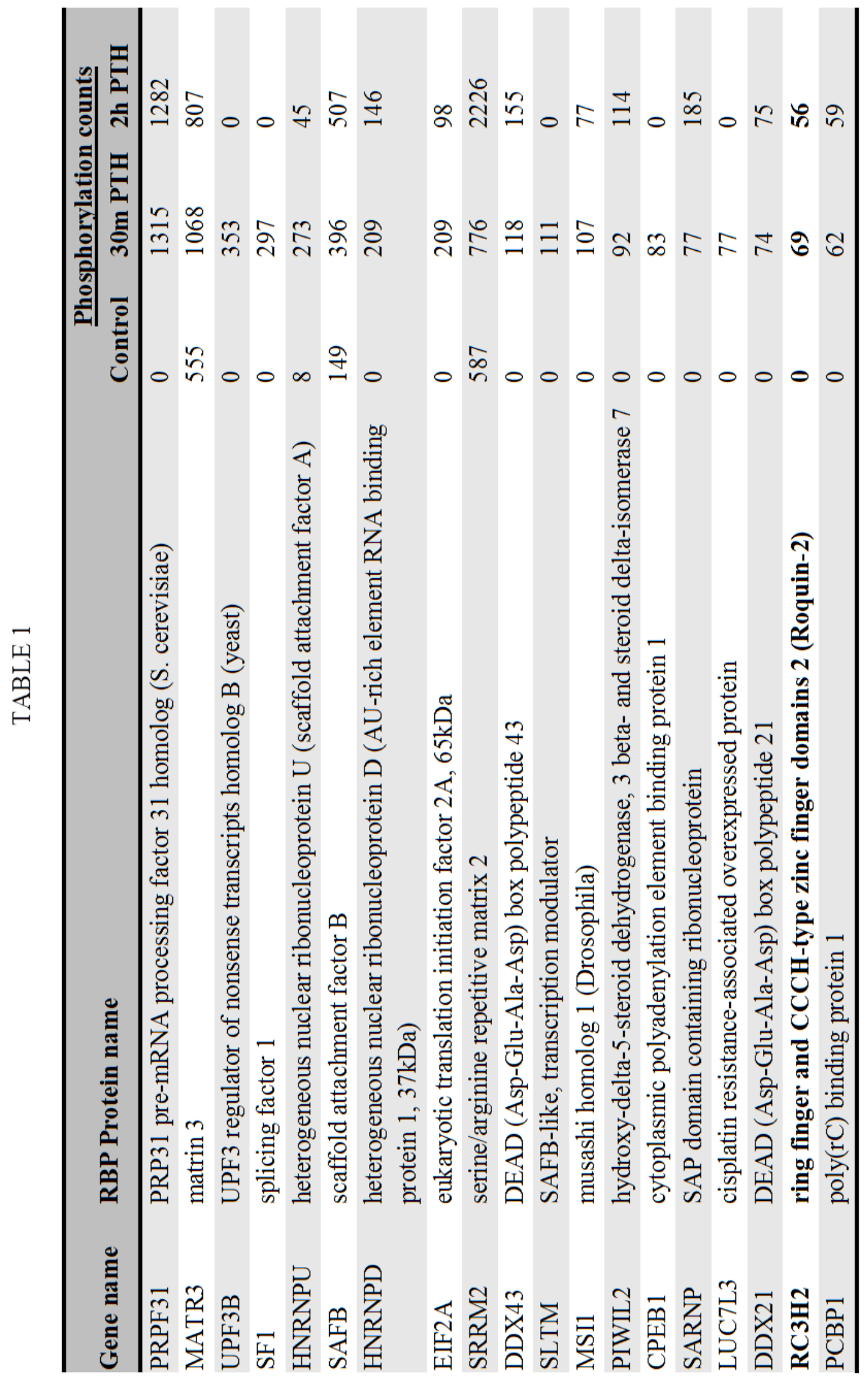




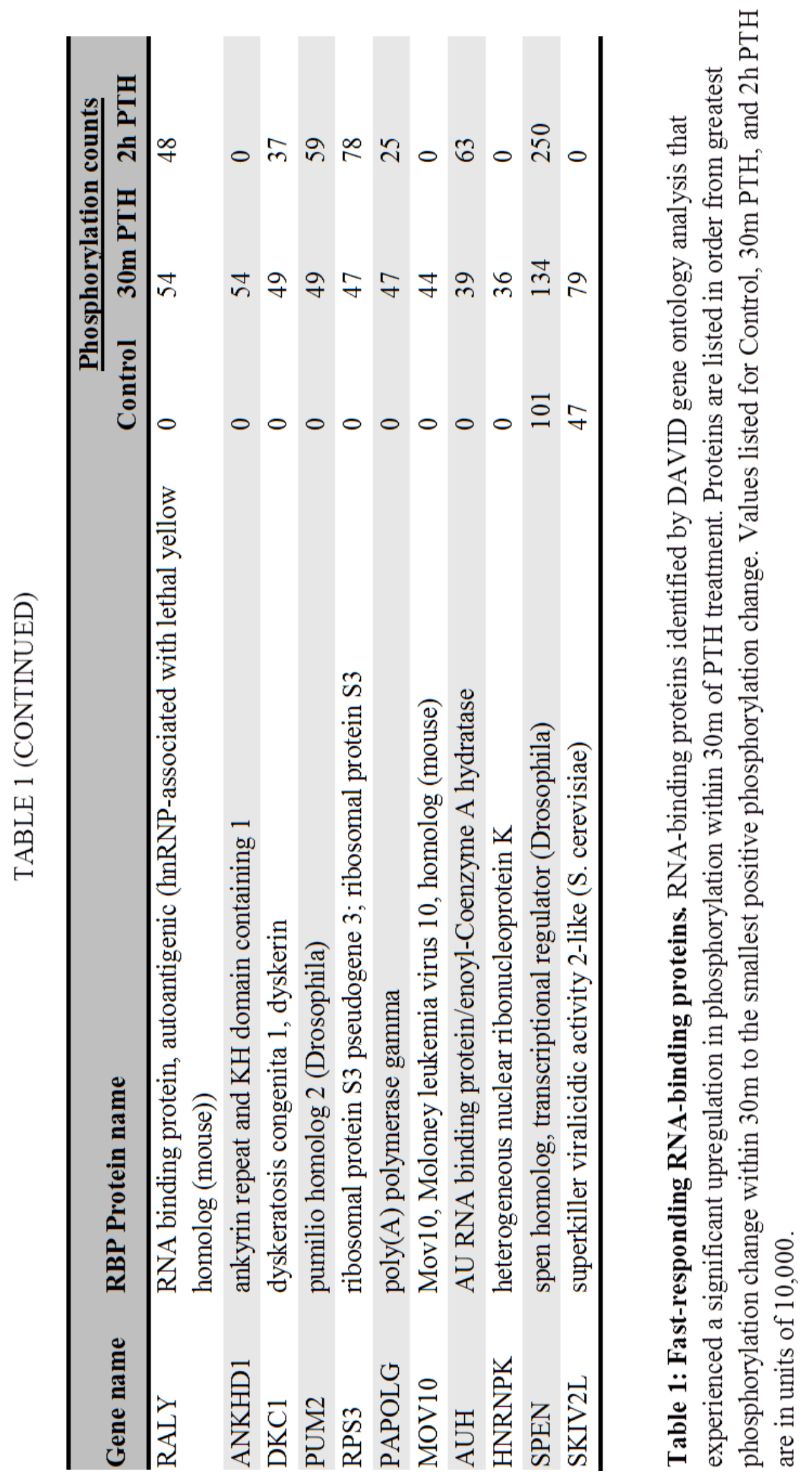




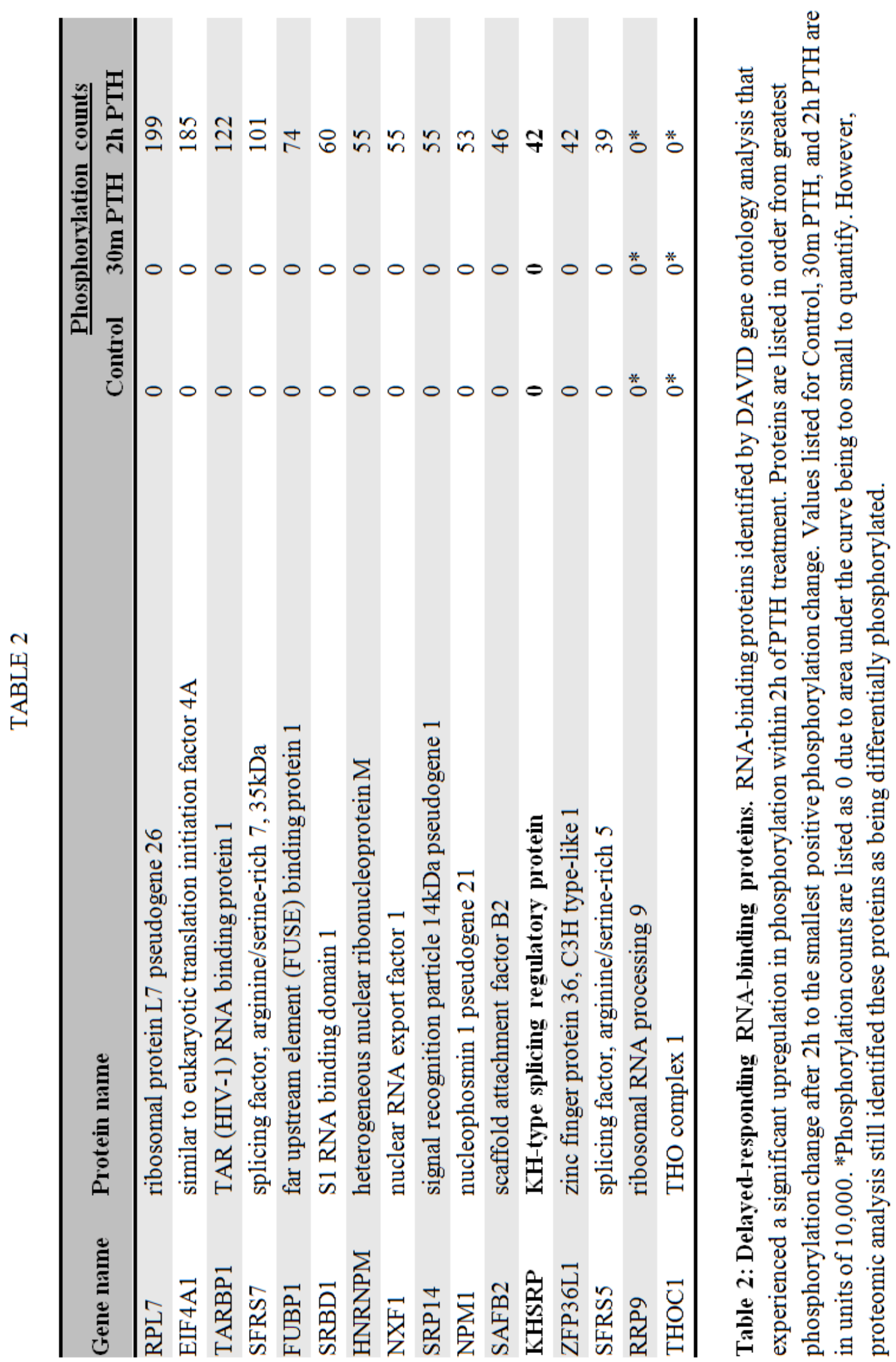




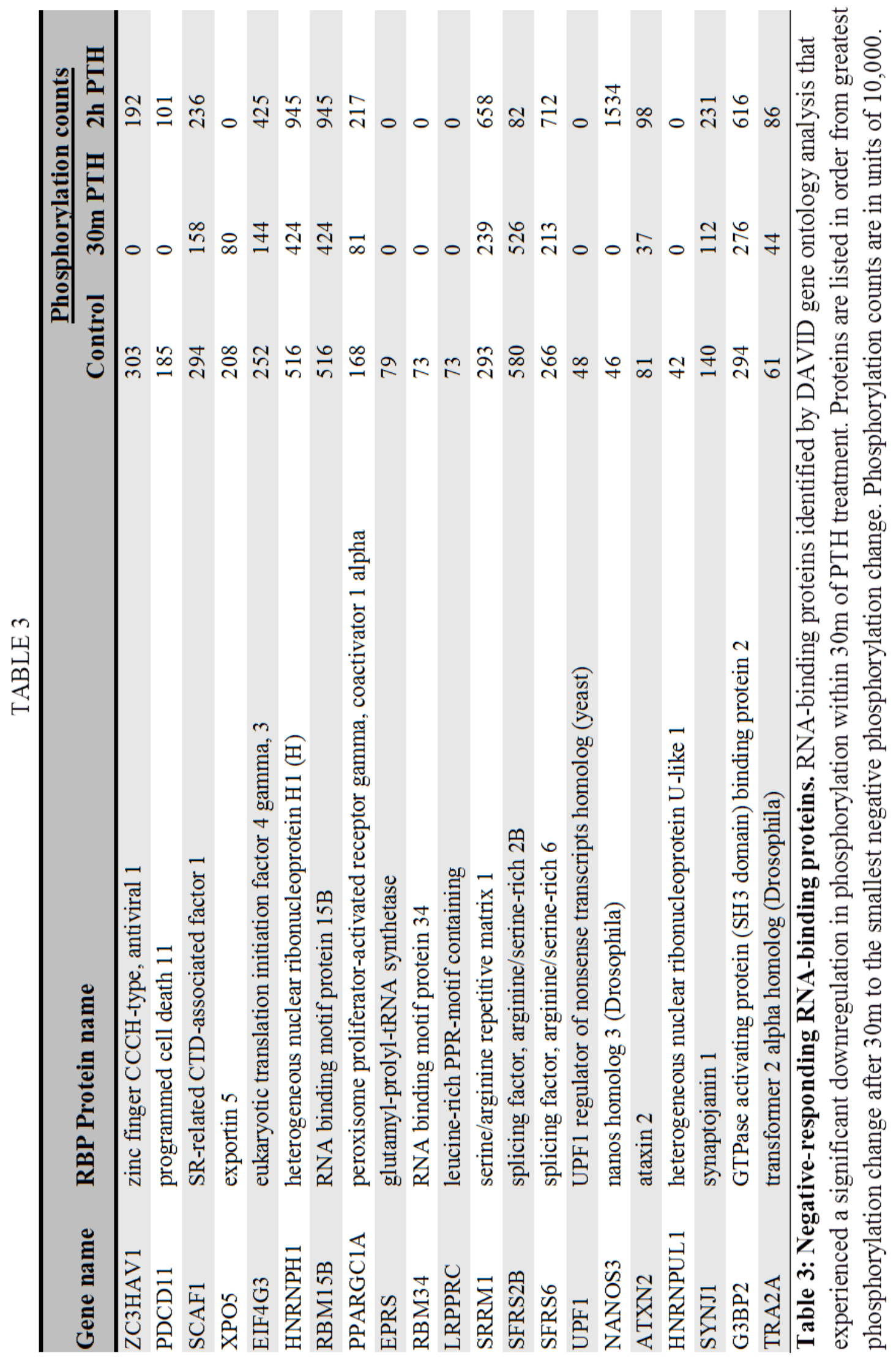




\section{Part 2: Regulation of KHSRP and Roquin-2 Expression by PTH}

Computational analysis of the PTH-responsive phosphoproteome suggests that two of the PTH-responsive RBPs, KHSRP and Roquin-2, are capable of interacting with the 3'UTR of Npt2a mRNA. To confirm the expression of KHSRP and Roquin-2 in the proximal tubule, cell fractions prepared from OK cells treated with and without PTH were subjected to Western blot analysis. As shown in Figure 20A, KHSRP expression was found in crude membrane, nuclear, and cytosolic fractions. KHSRP nuclear expression was also found to significantly increase in response to PTH. IHC of control and PTH-D1 mouse kidneys also confirmed proximal tubular expression of KHSRP (Figure 20B), and showed a nuclear expression pattern as well as cytosolic and/or intracellular compartment expression with distinct prominence on the basolateral side of the cell. Increased nuclear expression of KHSRP was observed in the PTH-D1 kidneys in comparison to control, supporting the results of the OK cell Western blot study.

To strengthen the hypothesis that PTH-regulated KHSRP expression and activity is related to the control of phosphate transport by PTH, and not another PTH-regulated function, we next treated OK cells with low phosphate media, a stimulus for Npt2a upregulation. We reasoned that if KHSRP is involved in destabilization of Npt2a mRNA expression and its expression increased with $\mathrm{PTH}$, then low phosphate should decrease its expression. Following $24 \mathrm{~h}$ treatment with low phosphate media, cell compartments were isolated by sucrose density gradient centrifugation, and KHSRP expression analyzed by Western blot. We observed decreased expression of KHSRP in the plasma membrane and endoplasmic reticulum in response to $24 \mathrm{~h}$ low phosphate, and increased KHSRP expression in the cytosol (Figure 20C). Interestingly, we also observed multiple 


\section{FIGURE 20}
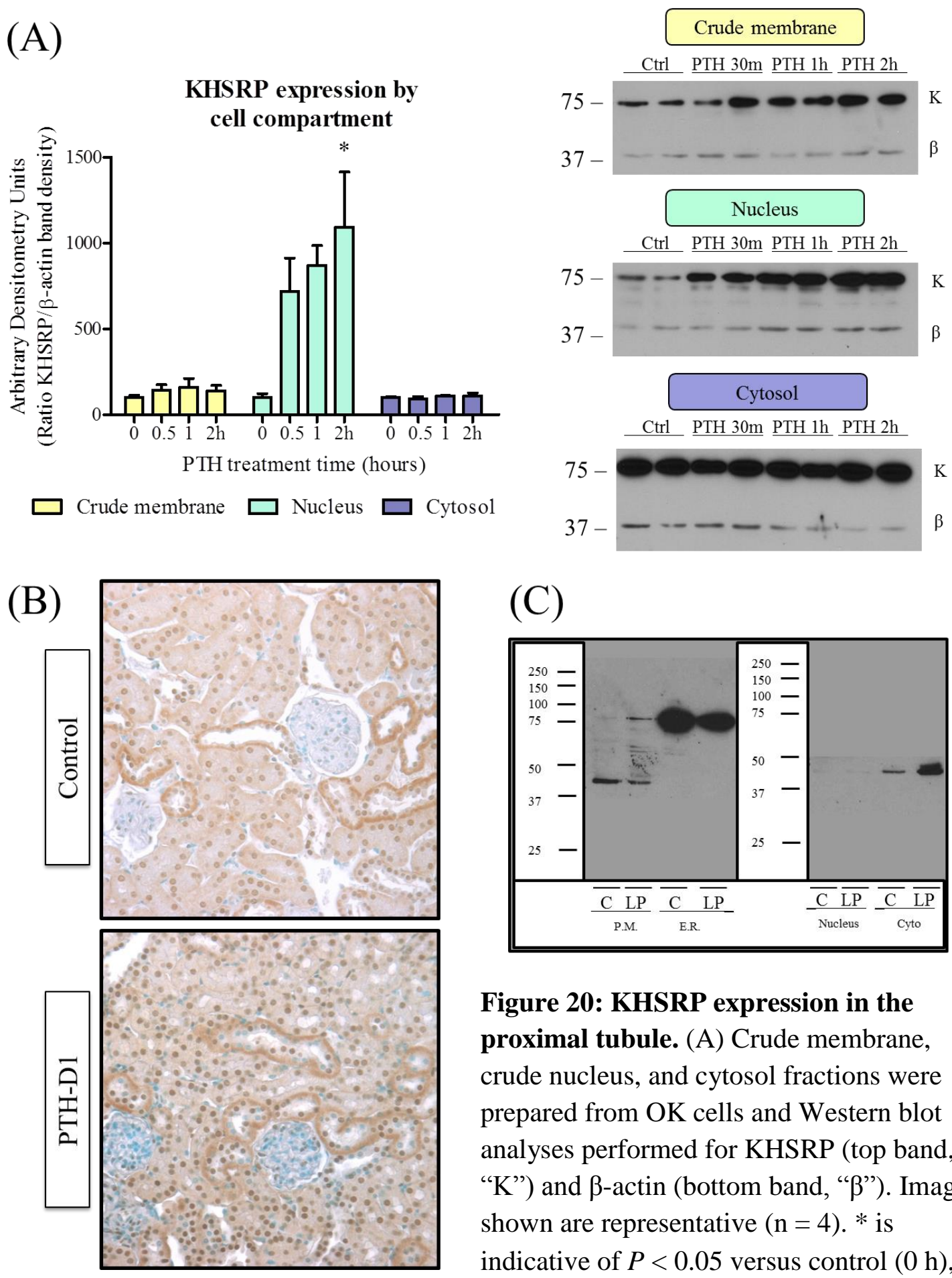

Figure 20: KHSRP expression in the proximal tubule. (A) Crude membrane, crude nucleus, and cytosol fractions were prepared from OK cells and Western blot analyses performed for KHSRP (top band, "K") and $\beta$-actin (bottom band, " $\beta$ "). Images shown are representative $(\mathrm{n}=4)$. $*$ is indicative of $P<0.05$ versus control $(0 \mathrm{~h})$, as

determined by one-way ANOVA. Percentages shown indicate relative changes of expression within each cell compartment. (B) Immunohistochemical staining for KHSRP in control FVB and PTH-D1 mouse kidneys. (C) Plasma membrane (P.M.), endoplasmic reticulum (E.R.), nucleus, and cytosol fractions were prepared from OK cells treated with regular or low phosphate media, and blotted for KHSRP. 
immunoreactive bands at different molecular weights than the typical $75 \mathrm{kD}$ band. Although we cannot conclusively comment on the functional importance of these potential variants, the presence of these bands with distinct cell compartment expression profiles indicates the possibility that KHSRP is expressed as several splice variants, with their functional role dependent on cellular distribution.

In addition to examining the effect of PTH on KHSRP expression, we also studied the response of Roquin-2 to PTH treatment. As shown in Figure 21A, we identified Roquin- 2 in the crude membrane, crude nucleus, and cytosolic fractions. PTH treatment over the course of $2 \mathrm{~h}$ did not significantly affect crude membrane Roquin-2 expression. However, in both the nuclear and cytosolic fractions, Roquin-2 expression increased. When OK cells were treated with low phosphate media for $24 \mathrm{~h}$, Roquin-2 expression decreased in both the endoplasmic reticulum and nuclear fractions (Figure 21B). As with KHSRP, we found that the molecular weight of Roquin-2 differed between cell compartments, with a smaller immunoreactive band observed in the E.R. fraction as opposed to the Roquin- 2 band in the nuclear fraction, again indicating the potential presence of compartment-specific splice variants.

\section{Part 3: Interaction of KHSRP and Roquin-2 with Npt2a mRNA}

We have established that the expression of both KHSRP and Roquin-2 is responsive to PTH and ambient phosphate, physiological regulators of proximal tubular phosphate reabsorption. Computational analysis predicts that both proteins can interact with the 3' UTR of Npt2a mRNA, but the expression studies alone do not provide conclusive evidence for a role of KHSRP or Roquin-2 in the destabilization of Npt2a 
(A)

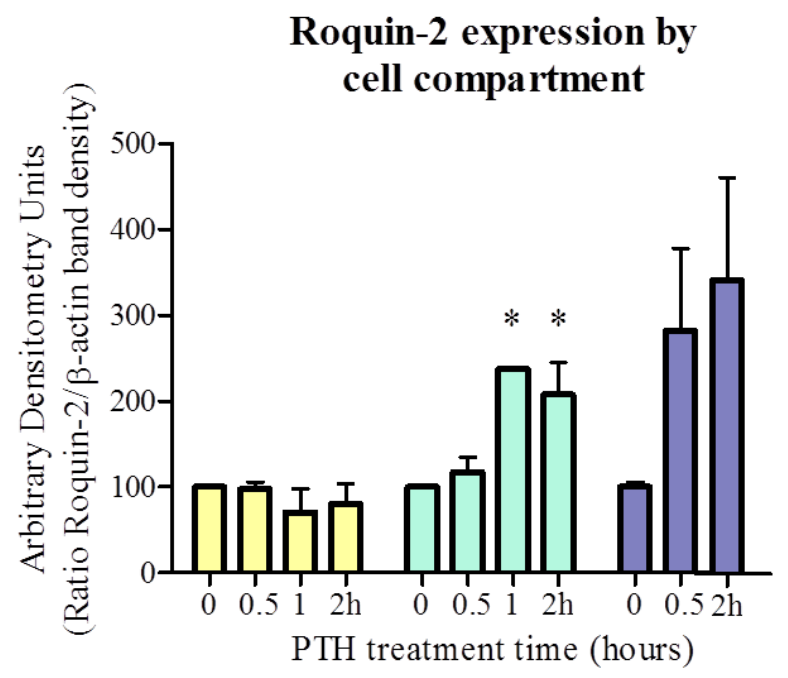

Crude membrane

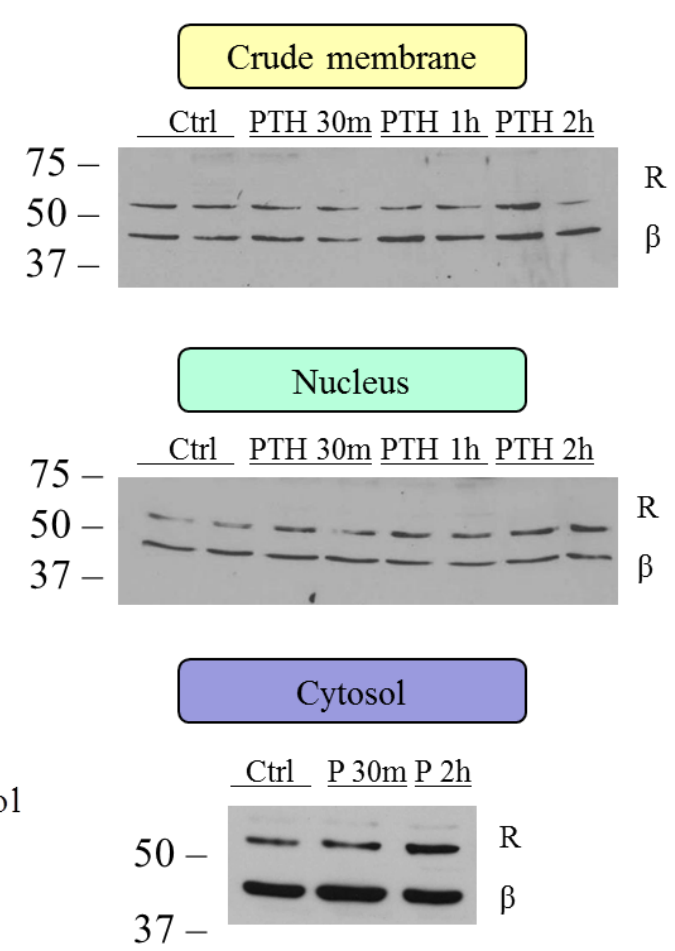

(B)

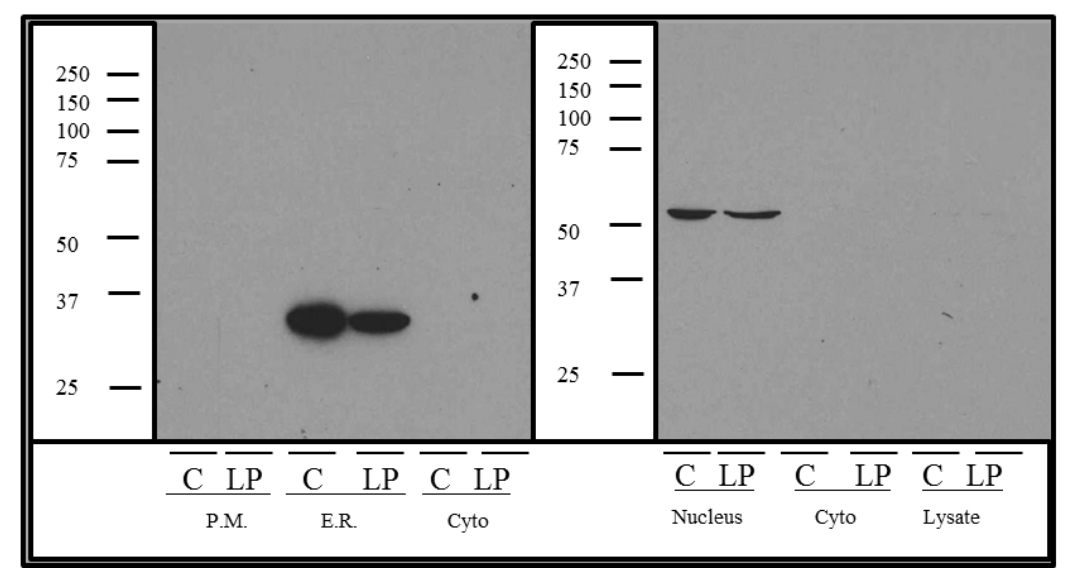

Figure 21: Roquin-2 expression in the proximal tubule. (A) Crude membrane, crude nucleus, and cytosol fractions were prepared from OK cells and Western blot analyses performed for Roquin-2 (top band, "R") and $\beta$-actin (bottom band, “ $\beta$ ”). Images shown are representative $(\mathrm{n}=4)$. * is indicative of $P<0.05$ versus control $(0 \mathrm{~h})$, as determined by one-way ANOVA. Percentages shown indicate relative changes of expression within each cell compartment. (B) Plasma membrane (P.M.), endoplasmic reticulum (E.R.), nucleus, and cytosol fractions were prepared from OK cells treated with regular or low phosphate media. Fractions were separated by sucrose density gradient centrifugation, separated by SDS-PAGE, and Western blotted for Roquin-2. 
mRNA. Therefore, to determine whether KHSRP and/or Roquin-2 interact with Npt2a mRNA, we next performed RNA-binding protein immunoprecipitation (RIP) assays, wherein an intact mRNP complex is pulled down with an antibody specific for an RBP, followed by RNA isolation and RT-qPCR to identify transcripts associated with the RBP. As shown in Figure 22A, immunoprecipitation of KHSRP pulled down Npt2a mRNA in comparison to isotype IgG IP. Immunoprecipitation of Roquin-2 also showed that Npt2a mRNA was pulled down with Roquin-2 (Figure 22B). While immunoprecipitation of KHSRP and Roquin-2 from PTH-treated cells showed less fold enrichment for Npt2a mRNA than in KHSRP and Roquin-2 IPs from control cells, normalization of Npt2a mRNA from the immunoprecipitation to Npt2a mRNA in the input will be necessary to determine whether more or less Npt2a mRNA is associated with the two RBPs in response to PTH treatment. 


\section{FIGURE 22}

(A)

Npt2a mRNA expression following

IP with KHSRP

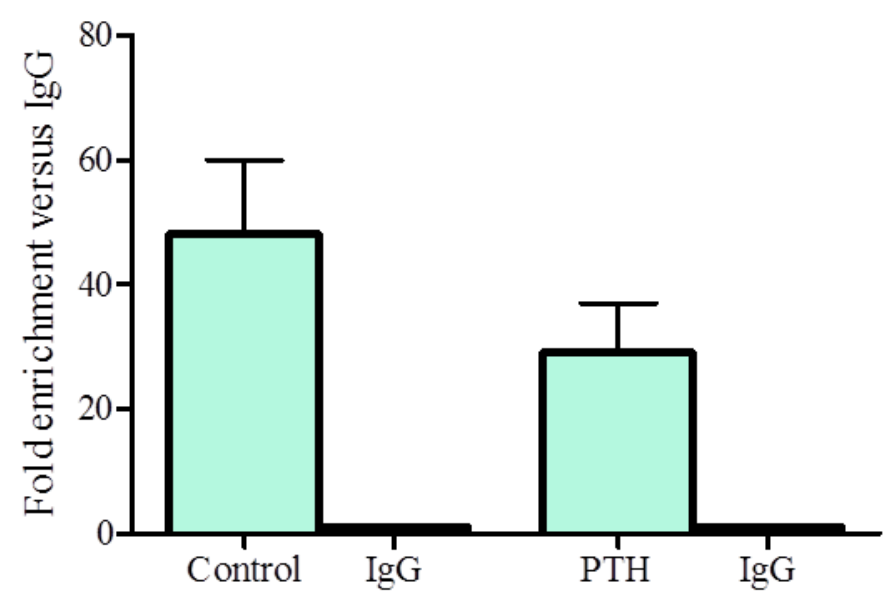

(B)

Npt2a mRNA expression following

IP with Roquin-2

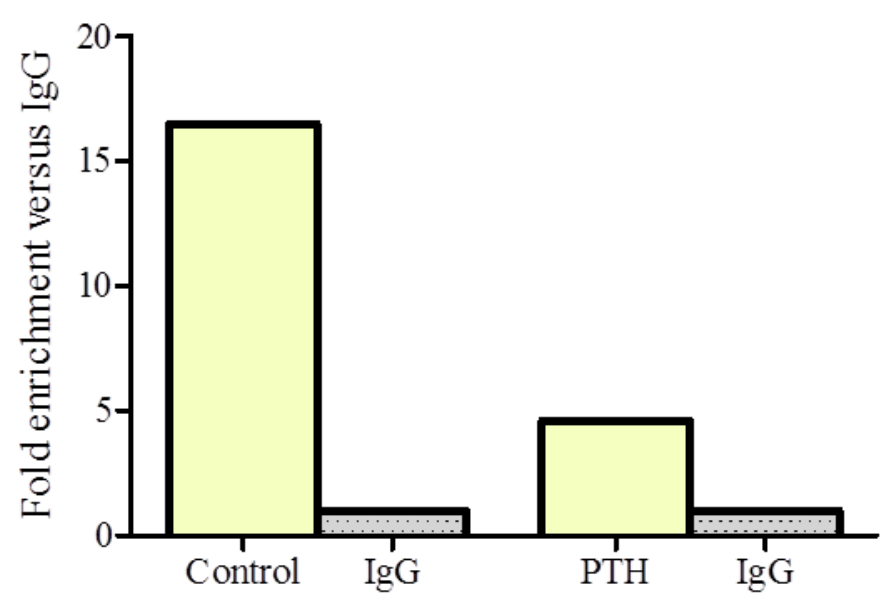

Figure 22: Interaction of KHSRP and Roquin-2 with Npt2a mRNA. IP of KHSRP or Roquin-2 followed by isolation of RNA and RT-qPCR was performed as described in Methods. Immunoprecipitation of (A) KHRSP ( $\mathrm{n}=3)$ and $(\mathrm{B})$ Roquin-2 $(\mathrm{n}=1)$ showed that Npt2a mRNA was pulled down with both RBPs. 


\section{$\underline{\text { DISCUSSION }}$}

For the first time, we have characterized the cell-wide phosphorylation events that occur in response to PTH. We were able to categorize 1139 out of the 1182 proteins that underwent significant changes in phosphorylation over the course of $2 \mathrm{~h}$ PTH treatment, and we discovered that $\mathrm{PTH}$, along with being a major regulator of proximal tubule ion transport, is also a major regulator of both transcription and translation. Almost one-fifth of the downstream targets of PTH are proteins involved in the process of transcription or its regulation. This category of biological processes altered by PTH was by far the most affected biological process, distantly followed by the categories of intracellular signaling cascade $(8.3 \%)$, phosphate metabolic process $(6.9 \%)$, and protein localization $(6.5 \%)$. As expected in characterizing the PTH-responsive phosphoproteome, we also found that PTH was also a major regulator of protein phosphorylation.

We found that PTH was also a major regulator of translation, as we identified 68 RNA-binding proteins whose phosphorylation status changed in response to PTH. Because DAVID was only able to map 812 of the submitted 1182 proteins to a molecular function, the number of RBPs regulated by PTH may be higher. Some of the RBPs modified by PTH-mediated signaling include members of the translation complex, such as EIF4G3, EIF2A, and EIF4A1. EIF4G3 is the linker protein between the 3' and 5' ends of mRNA, forming a closed-loop configuration that protects the mRNA from degradation by exonucleases ${ }^{161}$. PTH induces a transient dephosphorylation of EIF4G3, potentially affecting the stability of the closed-loop configuration and overall stability of the mRNP complex. 
The destabilization of Npt2a mRNA in response to PTH is a specific event, and likely related to modified RBP interaction with the 3'UTR. The destabilizing effect of PTH on Npt2a mRNA is a rapid process, as PTH decreases the level of Npt2a mRNA expression by $50 \%$ from over 8 hours under basal conditions to just over 2 hours. We focused on exploring the roles of Roquin-2 and KHSRP in mediating this process, as both were predicted to interact with the Npt2a 3'UTR.

KHSRP (hnRNPK-homology ( $\underline{\mathrm{KH}})$-type $\underline{\text { Splicing }} \underline{\text { Regulatory }}$ Protein) is a multifunctional RBP, and is involved not only in regulating mRNA stability, but also in mRNA localization and trafficking, pre-mRNA splicing, and miRNA maturation ${ }^{184}$. KHSRP mediates the rapid shortening of the poly(A) tail accompanied by 3' -5 ' endonucleolytic decay by recruiting exosome machinery, including the enzymes poly(A) RNase (PARN), exosome component EXOSC2, and DCP2, the decapping enzyme $^{183,185,186}$. KHSRP contains four KH domains, and all four domains are necessary for interaction with the decay-promoting exosome ${ }^{187}$. It is through these $\mathrm{KH}$ domains that KHSRP recognizes AREs in 3'UTRs of transcripts ${ }^{184}$. While the 3'UTR of Npt2a mRNA lacks the canonical AUUUA element, it nonetheless has type III AREs within U-rich regions, and two predicted binding sites for KHSRP. Both predicted binding sites for KHSRP within the 3' UTR of Npt2a mRNA consist of the sequence GUCC, with one of the elements starting at base 7 (from the $3^{\prime}$ end) and the other starting at base $176^{172}$. The first binding site is situated very closely to the $3^{\prime}$ end of the mRNA (Figure 18C), which is predicted to be right next to the poly(A) tail of the mature transcript. This proximity in theory would facilitate the actions of recruited deadenylases to promote mRNA decay, 
although it remains to be seen whether the 3 ' binding motif is important for promoting destabilization.

Much less is known about the function of Roquin-2 as an mRNA decay factor in comparison to the studies that have examined KHSRP-mediated RNA degradation. Loss of function mutations in Roquin are known to lead to a severe lupus-like autoimmune syndrome in mice ${ }^{188,189}$. Roquin- 2 and its paralog Roquin- 1 have redundant functions in $\mathrm{T}$ cells, and Roquin-2 can fully compensate for the loss of Roquin- $1^{189}$. Because of this prominent systemic role of Roquin-2, the vast majority of Roquin-2 studies have focused on its effects on the immune system, and no studies to date have examined the role of Roquin-2 in the kidney. However, several studies have examined the mechanisms of RNA binding and promotion of decay by Roquin-2, and these mechanisms of mRNA regulation are likely to be conserved in other tissues. Roquin-2 recognizes and binds 3'UTR cis-elements referred to as constitutive decay elements (CDEs), which are a conserved class of stem-loop motifs typically found in regulators of development and inflammation ${ }^{181}$. Upon binding, Roquin-2 induces mRNA decay by promoting a deadenylase complex ${ }^{181}$. Roquin- 2 has two binding sites for RNA - one that recognizes stem-loop motifs, and another that recognizes double-stranded RNA - and both are required for promoting RNA decay ${ }^{190}$. We could not identify the conserved CDE stemloop motif in the opossum 3' UTR of Npt2a, but we did find a CDE motif in the mouse 3'UTR. While interaction with RNA requires a preserved CDE consensus in a stem-loop motif, recent studies suggest that Roquin-RNA binding is more dependent on secondary structures and non-sequence-specific contacts, implying that a broader spectrum of RNAs than previously thought may interact with the decay factor ${ }^{191}$. 
The studies within this chapter provide evidence that KHSRP and Roquin-2 are potential modulators of Npt2a mRNA stability. We showed that Npt2a mRNA was pulled down with both KHSRP and Roquin-2, which strongly suggests that both RBPs interact with the Npt2a transcript. Additionally, KHSRP and Roquin-2 expression is modulated by known regulators of Npt2a expression. Western blot showed a 10-fold increase in the nuclear expression of KHSRP after $2 \mathrm{~h}$ PTH. This increase was also observed in the nuclei of PTH-D1 mouse proximal tubules in comparison to age-matched control mice, as determined through immunohistochemistry. While PTH treatment increased the nuclear expression of KHSRP, treatment of OK cells with low phosphate media, a stimulus for Npt2a upregulation, decreased both the plasma membrane and endoplasmic reticulum expression of KHSRP, and increased the cytosolic expression. Perhaps more interesting than the changes in expression are the differences in the molecular weight of KHSRP dependent on cellular location. Normally a $75 \mathrm{kDa}$ protein, the predominant KHSRP band in both the plasma membrane and cytosolic compartments was around 40 $\mathrm{kDa}$. In the cytosolic fraction, low phosphate treatment stimulated an increase in KHSRP, and the appearance of a doublet in only the low-phosphate-treated condition suggests an increase in KHSRP phosphorylation. We also observed a shift in molecular weight in the plasma membrane compartment in response to low phosphate, from $40 \mathrm{kDa}$ up to $75 \mathrm{kDa}$. These differences in the molecular weight of KHSRP likely indicate the expression of KHSRP splice variants, with potentially varying functions based on cell distribution. In the human genome, there are 10 known protein-coding splice variants of KHSRP ${ }^{177,192}$. However, further studies are required to properly interpret the functional significance of these changes in the OK cell. 
The changes in Roquin-2 expression in response to PTH and low phosphate differed slightly from those of KHSRP. Whereas PTH induced only nuclear increases in KHSRP expression, Roquin- 2 expression in response to $2 \mathrm{~h}$ PTH increased in both the cytosolic and nuclear compartments by $342 \%$ and $207 \%$, respectively. Separation of OK cell compartments by sucrose density gradient centrifugation following low phosphate treatment showed decreased expression of Roquin- 2 in the E.R. and nucleus in response to 24h low phosphate. As observed with KHSRP, different molecular weights for Roquin- 2 were found in the E.R. and nucleus. The typical band for Roquin-2 in the OK cell is approximately $55 \mathrm{kDa}$, which is the size found in the nucleus, but E.R. Roquin-2 is approximately $35 \mathrm{kDa}$. Again, these results may imply that Roquin- 2 is expressed as several different splice variants, with differential functions of the Roquin-2 variants based on organelle location. Human Roquin-2 is known to be expressed as 7 different proteincoding splice variants ${ }^{177,192}$, and it is likely that opossum Roquin-2 is expressed as several splice variants as well.

The studies regarding KHSRP and Roquin-2 expression in response to stimuli that regulate $\mathrm{Npt} 2 \mathrm{a}$ expression are intriguing, and warrant further study to determine whether the interaction of those two RBPs with Npt2a mRNA serves to regulate Npt2a expression. Both KHSRP and Roquin-2 display an overall increase in expression in response to PTH and an overall decrease in expression in response to low phosphate. This inverse relationship to Npt2a expression indicates that KHSRP and Roquin-2 may act as destabilizing trans-factors for the Npt2a transcript. Interestingly, KHSRP modulates PTH mRNA decay rates in the parathyroid gland in response to changes in serum calcium or phosphate. This occurs through the interaction of KHSRP with type III AU-rich elements 
in the PTH 3'UTR, which does not contain the classical AUUUA pentameric motif ${ }^{164,182}$. These are the same putative KHSRP-binding motifs found in the 3'UTR of Npt2a, and this relationship may indicate that KHSRP has a broader systemic role in the regulation of calcium and phosphate homeostasis.

We have shown that KHSRP and Roquin-2 are responsive to PTH, with changes in both phosphorylation and expression. However, protein phosphorylation can either increase or decrease the activity of a protein, and we cannot make any conclusive statements about whether phosphorylation of Roquin-2 and KHSRP by PTH signaling upregulates or downregulates their decay-promoting function. Nothing is currently known about how post-translational modifications such as phosphorylation affect Roquin-2 activity. Several studies have, however, examined KHSRP activity in response to phosphorylation events. Known KHSRP phosphorylation events are induced by both the MAPK p38 and Akt/PKB pathways ${ }^{193}$. Phosphoproteomic analysis showed that PTH induces KHSRP phosphorylation at S181. S181 is within the K1 domain, one of the four $\mathrm{KH}$ domains required for binding RNA and recruiting exosomes. Although the functional significance of this particular modification is unknown, there are several studies that have addressed the effect of the phosphorylation of the neighboring S193 residue on KHSRP function. Akt mediates S193 phosphorylation, which promotes the unfolding of the K1 domain, leading to decreased ARE binding and accumulation in the nucleus ${ }^{193-195}$. Although we have not addressed the change in KHSRP function induced by PTH, the phosphorylation of S181, which is also within the K1 domain, potentially has the same destabilizing effect on the domain structure as the phosphorylation of S193. Therefore, PTH may decrease KHSRP activity. While this action would typically stabilize KHSRP 
target transcripts, several studies have shown that KHSRP can also mediate mRNA stabilization. Because we have not performed in vitro degradation assays of Npt2a mRNA in the presence and absence of KHSRP, we cannot currently make any conclusive statements about the role of KHSRP in regulating Npt2a mRNA stability. Further experiments are required to define the exact function of KHSRP and Roquin-2 in promoting Npt2a mRNA decay.

\section{CONCLUSION}

We conclude that PTH is a major regulator of transcription and translation in the proximal tubule. The RNA-binding proteins KHSRP and Roquin-2 interact with Npt2a mRNA, and are potential mediators of PTH-induced mRNA destabilization. 


\section{CHAPTER VII}

\section{DISCUSSION: BROADENED BASES AND FUTURE DIRECTIONS}

The major impact of the work described within this dissertation lies in the establishment and description of an additional and alternative pathway for the regulation of Npt2a expression by PTH in the renal proximal tubule. As Npt2a expression is a major determinant of renal phosphate handling, total body phosphate homeostasis, and serum phosphate levels, the elucidation of newer mechanisms for the regulation of Npt2a by PTH holds promise for novel applications in clinical medicine. Because serum phosphate levels correlate with cardiovascular risk, understanding the determinants of serum phosphate may have important implications for the prevention and treatment of cardiovascular disease, the major cause of death in this country.

In Chapter IV, we discovered that PTH post-transcriptionally regulates Npt2a expression through destabilization of the Npt2a transcript, an effect that was dependent on both transcription and translation. In Chapter V, we observed that both the PTHinduced PKA and PKC pathways contribute to PTH-mediated Npt2a mRNA destabilization. PKA activation appears to produce early changes in downstream effectors that provide sustained downregulation of Npt2a mRNA, and PKC activation produces later effects that promote mRNA decay after the initial period of PKA-induced destabilization. Chapter VI described the cell-wide phosphorylation events that occur in 
response to PTH. We were able to identify two PTH-responsive RBPs, Roquin-2 and KHSRP, that bind to Npt2a mRNA and potentially regulate its stability in response to PTH.

From these studies, we propose the following model of PTH-induced Npt2a destabilization (Figure 23): PTH stimulation of the PTH receptor activates both the PKA and PKC signaling pathways. PKA actions produce early and sustained downstream effects that lead to Npt2a mRNA destabilization, whereas PKC signaling contributes downstream signaling that acts during the latter phase of destabilization to sustain the phosphaturic effects of PTH. These signaling events lead to the increased expression and phosphorylation of Roquin-2 and KHSRP, which target Npt2a mRNA to expose the 3'UTR and alter the stability of the basal Npt2a mRNP complex. Binding of KHSRP and/or Roquin-2 destabilizes the complex and promotes decay, potentially through exosome or decapping enzyme recruitment, followed by either deadenylation, exonucleolytic cleavage, endonucleolytic cleavage, or some combination thereof. Thus, we conclude that PTH post-transcriptionally regulates Npt2a expression through PKAand PKC-dependent alteration of the stability of the Npt2a mRNP complex, promoting mRNA decay.

Although we have provided evidence to support this conclusion, there are additional experiments that will be necessary to confirm our findings. While we have data that show that both Roquin-2 and KHSRP bind to Npt2a mRNA, and that both RBPs are responsive to physiological stimuli that regulate phosphate reabsorption, we have not conclusively shown that they mediate Npt2a mRNA decay in response to PTH. To determine their contribution to PTH-mediated Npt2a mRNA destabilization, knockdown 
studies of Roquin-2 and KHSRP should be performed. If either or both RBPs are involved in regulating Npt2a mRNA decay in response to PTH, then silencing their expression should abolish or at least diminish PTH-mediated Npt2a mRNA destabilization.

\section{FIGURE 23}

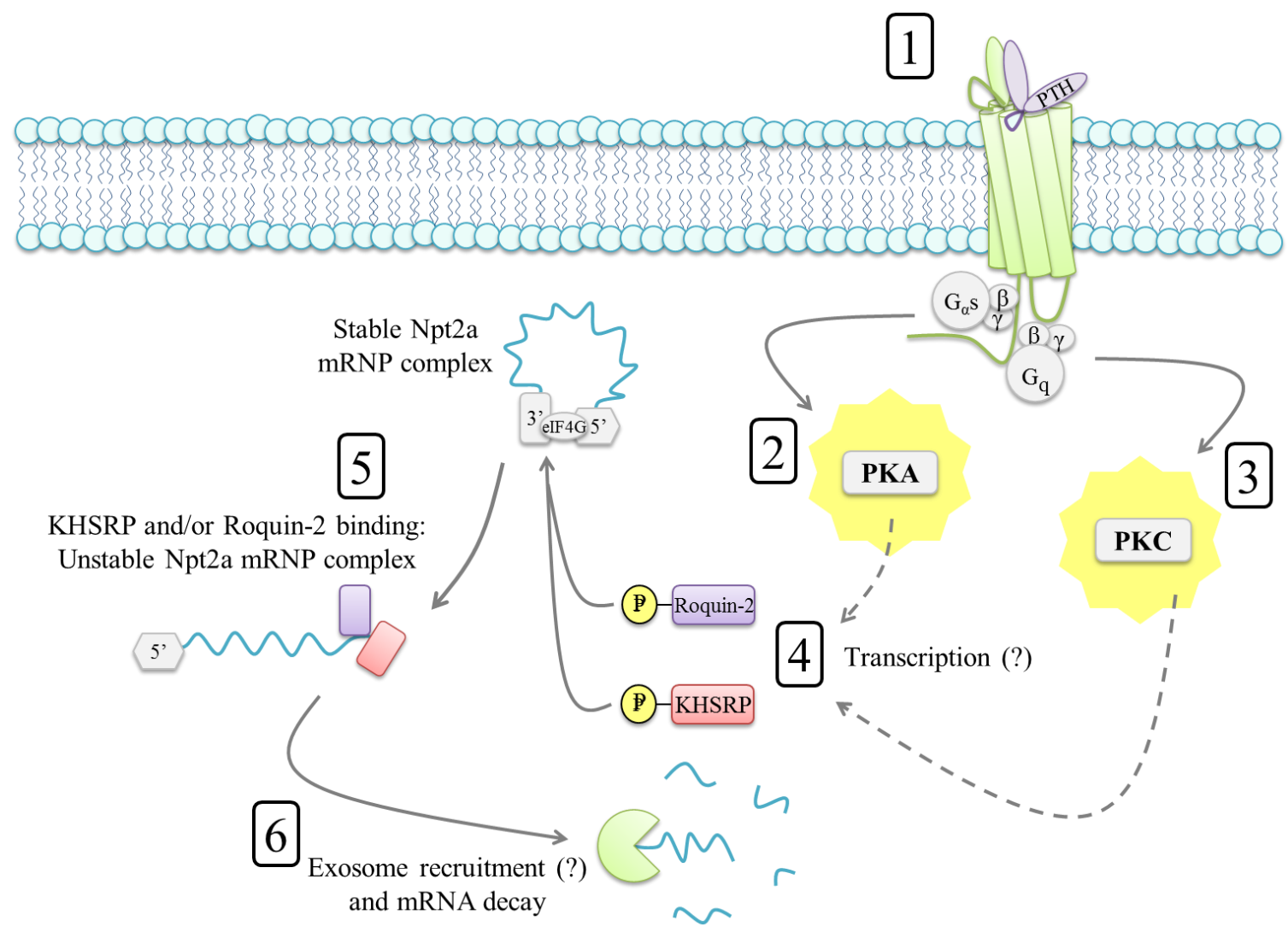

Figure 23: Proposed model of PTH-mediated Npt2a mRNA destabilization. (1) PTH(1-34) binds the PTH receptor. (2) PTHR1 stimulation activates PKA, which produces early phosphaturic responses to PTH. (3) PKA activation is followed by PKC activation, which produces later effects to sustain PTH-induced phosphaturia. (4) Activation of second messenger signaling cascades alters the expression and phosphorylation status of the RBPs Roquin-2 and KHSRP. (5) Altered RBP composition of the Npt2a mRNP complex through differential binding/recruitment activities of Roquin-2 and/or KHSRP interrupts the closed-loop conformation, producing an unstable Npt2a mRNA complex, which leads to (6) exosome recruitment (potentially) and Npt2a mRNA decay. 
Also of interest in further exploring the contribution of Roquin-2 and KHSRP to the suppression of Npt2a expression are the changes in the cellular distribution of the two RBPs, and how those changes affect mRNA decay. We observed significant nuclear expression of KHSRP in response to PTH, and significant cytosolic and nuclear expression of Roquin-2. While some studies suggest that phosphorylation of KHSRP at different sites from the PTH-responsive serine residue promotes nuclear retention of the RBP and decreased mRNA destabilization, in neither OK cells nor mouse proximal tubules we did not observe a coordinate decrease in the cytosolic expression of KHSRP in response to $\mathrm{PTH}$, suggesting that $\mathrm{PTH}$-induced phosphorylation may produce different effects on KHSRP activity than has been previously reported. Roquin-2 expression, on the other hand, displayed a time-dependent increase in both the cytosol and the nucleus. Nothing is currently known about the effects of phosphorylation on Roquin-2 activity, and thus it would be beneficial to construct phosphorylation-deficient and -mimicking mutants of both KHSRP and Roquin-2 and examine how that alters the PTH responses regarding Npt2a mRNA in the proximal tubule.

In addition to looking at the functional significance of Roquin-2 and KHSRP phosphorylation, future studies directed towards identifying the kinase responsible for their phosphorylation in response to PTH will further clarify the contribution of the PKA and PKC pathways to Npt2a mRNA decay. Our current studies suggest that the rapid and sustained stimulation of Roquin-2 phosphorylation may be in response to early PKA activity, whereas the later activation of KHSRP may be due to the prolonged signaling generated by PKC. Phosphoproteomic analysis identified residue S181 on KHSRP and residues T543 and S549 on Roquin-2 as being phosphorylated in response to PTH. Using 
ScanSite to identify canonical kinase motifs within KHSRP and Roquin- $2^{196}$, we found several kinases that were predicted to phosphorylate the particular residues responsive to PTH. Within Roquin-2, T543 is a canonical binding site for both Akt and 14-3-3 mode 1, whereas S549 is a putative phosphorylation site for GSK3 kinase, Erk1 kinase, and p38 MAP kinase. Within KHSRP, S181 is predicted to be phosphorylated by calmodulindependent kinase 2, CDK motif 1, and CDK motif 2. Although neither PKA nor PKC were predicted to directly modify the PTH-responsive sites on the RBPs, it is possible that PTH stimulates phosphorylation of other residues within the protein that were not detected by mass spectrometry, as both PKA and PKC were predicted to phosphorylate other residues within Roquin-2 and KHSRP. It is also possible that PTH stimulates the indirect activation of the aforementioned kinases through the activation of PKA and PKC. Further clarification would come from exploring the contribution of other PTHactivated signaling pathways, both dependent and independent of PKA and PKC activation, to the overall effect on RBP activity and Npt2a mRNA stability.

As we identified KHSRP as a potential mediator of PTH-induced Npt2a mRNA degradation, it is interesting to note that KHSRP has been previously characterized as a regulator of PTH mRNA stability in the parathyroid gland. The further exploration of the role of KHSRP in regulating proximal tubular phosphate transport may allow for the discovery of common mechanisms for the regulation of mineral metabolism throughout multiple tissues in the body. While we chose to initially study the involvement of Roquin-2 and KHSRP in PTH-mediated Npt2a mRNA destabilization, other RBPs in the PTH-responsive phosphoproteome were also categorized by DAVID analysis as regulators of mRNA stability. These proteins include ZFP36L1, HNRNPD, and 
HNRNPU. While HNRNPD and HNRNPU are ubiquitously expressed and not likely to be specific for PTH-induced Npt2a mRNA regulation, ZFP36L1 is another candidate protein that could possibly be involved in the regulation of Npt2a mRNA. Like KHSRP, the phosphorylation status of ZFP36L1 was increased after 2 hours of PTH stimulation, and it was also predicted by RPISeq to interact with the 3' UTR of Npt2a mRNA. Thus, future experiments examining the potential role of ZFP36L1 in modulating Npt2a mRNA stability may yield promising results. Although we have already identified two PTHresponsive RBPs that may regulate Npt2a mRNA stability, there are multiple RBPbinding sites within the 3'UTR of Npt2a mRNA, and differential regulation of several RBP interactions, not just one, may be required for the full decay effect.

The fact that over 200 regulators and effectors of transcription are altered in response to PTH lends credence to our model of PTH-induced protein expression mediating Npt2a mRNA destabilization. While the decay response is rapid, PTHmediated downregulation of Npt2a mRNA and protein expression appears to reach a steady state. Chronic PTH stimulation greatly diminishes the apical expression of Npt2a, but it does not eliminate it entirely. Similarly, chronic PTH stimulation greatly diminishes Npt2a mRNA levels, but again, this effect reaches maximal suppression around 12h, achieving a new steady state of expression. We have identified the cellular responses that mediate the initial reduction in Npt2a mRNA, but further work remains to elucidate the chronic changes in the Npt2a mRNP complex that contribute to a rate of Npt2a mRNA decay equal to the rate of Npt2a mRNA synthesis.

We have answered several questions about the mechanisms behind the chronic downregulation of proximal tubular phosphate reabsorption by $\mathrm{PTH}$, but we have also 
generated many more. A century's worth of research on the processes underlying the regulation of phosphate homeostasis has brought us to our current understanding, and yet the field of knowledge still remains incomplete. Within these chapters, however, we have expanded that knowledge base through the identification of a novel mechanism of Npt2a regulation by $\mathrm{PTH}$, and we have provided a basis for continuing to explore the physiological mechanisms underlying proximal tubular phosphate reabsorption. 


\section{REFERENCES}

1. Bowler MW, Cliff MJ, Waltho JP, Blackburn GM. Why did Nature select phosphate for its dominant roles in biology? New J. Chem. 2010;34(5):784. doi:10.1039/b9nj00718k.

2. Penido MGMG, Alon US. Phosphate homeostasis and its role in bone health. Pediatr. Nephrol. 2012;27(11):2039-48. doi:10.1007/s00467-012-2175-z.

3. Moz Y, Silver J, Naveh-Many T. Protein-RNA Interactions Determine the Stability of the Renal NaPi-2 Cotransporter mRNA and Its Translation in Hypophosphatemic Rats. J. Biol. Chem. 1999;274:25266-25272.

4. Taylor CW, Tovey SC. From parathyroid hormone to cytosolic $\mathrm{Ca} 2+$ signals. Biochem. Soc. Trans. 2012;40(1):147-52. doi:10.1042/BST20110615.

5. Faroqui S, Levi M, Soleimani M, Amlal H. Estrogen downregulates the proximal tubule type IIa sodium phosphate cotransporter causing phosphate wasting and hypophosphatemia. Kidney Int. 2008;73(10):1141-50. doi:10.1038/ki.2008.33.

6. Haussler MR, Whitfield GK, Kaneko I, et al. The role of vitamin D in the FGF23, klotho, and phosphate bone-kidney endocrine axis. Rev. Endocr. Metab. Disord. 2012;13(1):57-69. doi:10.1007/s11154-011-9199-8.

7. Baker SB, Worthley LIG. The essentials of calcium, magnesium and phosphate metabolism: part I. Physiology. Crit. Care Resusc. 2002;4(4):301-6. Available at: http://www.ncbi.nlm.nih.gov/pubmed/16573443.

8. Hedbäck G, Odén a. Increased risk of death from primary hyperparathyroidism--an update. Eur. J. Clin. Invest. 1998;28(4):271-6. Available at:

http://www.ncbi.nlm.nih.gov/pubmed/9615902.

9. Raue F. Increased incidence of cardiovascular diseases in primary hyperparathyroidism--a cause for more aggressive treatment? Eur. J. Clin. Invest. 1998;28(4):277-8. Available at: http://www.ncbi.nlm.nih.gov/pubmed/9615903. 
10. Conzo G, Perna A, Candela G, et al. Long-term outcomes following "presumed" total parathyroidectomy for secondary hyperparathyroidism of chronic kidney disease. G Chir 2012;33:379-382.

11. Bansal VK. Serum Inorganic Phosphorus. In: Walker HK, Hall WD, Hurst JW, eds. Clinical Methods: The History, Physical, and Laboratory Examinations. 3rd Edition. 3rd ed. Boston; 1990:895-899.

12. Menon MC, Ix JH. Dietary phosphorus, serum phosphorus, and cardiovascular disease. Ann. N. Y. Acad. Sci. 2013;1301:21-6. doi:10.1111/nyas.12283.

13. Frazão JM, Braun J, Messa P, Dehmel B, Mattin C, Wilkie M. Is serum phosphorus control related to parathyroid hormone control in dialysis patients with secondary hyperparathyroidism? BMC Nephrol. 2012;13:76. doi:10.1186/14712369-13-76.

14. Shroff R. Phosphate is a vascular toxin. Pediatr. Nephrol. 2013;28(4):583-93. doi:10.1007/s00467-012-2347-x.

15. Chen NX, O’Neill KD, Duan D, Moe SM. Phosphorus and uremic serum upregulate osteopontin expression in vascular smooth muscle cells. Kidney Int. 2002;62(5):1724-31. doi:10.1046/j.1523-1755.2002.00625.x.

16. Shioi A, Nishizawa Y, Jono S, Koyama H, Hosoi M, Morii H. BetaGlycerophosphate Accelerates Calcification in Cultured Bovine Vascular Smooth Muscle Cells. Arterioscler. Thromb. Vasc. Biol. 1995;15(11):2003-2009. doi:10.1161/01.ATV.15.11.2003.

17. Takeda E, Taketani Y, Sawada N, Sato T, Yamamoto H. The regulation and function of phosphate in the human body. Biofactors 2004;21((1-4)):345-355.

18. Allen DG, Westerblad H. Role of phosphate and calcium stores in muscle fatigue. J. Physiol. 2001;536(Pt 3):657-65. Available at:

http://www.pubmedcentral.nih.gov/articlerender.fcgi?artid=2278904\&tool=pmcen trez\&rendertype $=$ abstract.

19. Yang HT, Yim H, Cho YS, et al. Change of serum phosphate level and clinical outcome of hypophosphatemia in massive burn patient. J. Trauma Acute Care Surg. 2012;73(5):1298-302. doi:10.1097/TA.0b013e3182701e09.

20. Renkema KY, Alexander RT, Bindels RJ, Hoenderop JG. Calcium and phosphate homeostasis: concerted interplay of new regulators. Ann. Med. 2008;40(2):82-91. doi:10.1080/07853890701689645. 
21. Bagga A, Hari P, Vasudev AS, Sharma A, Srivastava RN. Hypophosphatemic Rickets with Hypercalciuria. Indian Pediatr. 1995;32(November).

22. Mason J. Vitamins, trace minerals, and other micronutrients. In: Goldman L, Ausiello D, eds. Cecil Medicine. 24th ed. Philadelphia, PA: Saunders Elsevier; 2011.

23. Ikeda K, Takeshita S. Factors and mechanisms involved in the coupling from bone resorption to formation: how osteoclasts talk to osteoblasts. J. bone Metab. 2014;21(3):163-7. doi:10.11005/jbm.2014.21.3.163.

24. Agus ZS, Puscttrr JB, Senesky D, Goldberg M. Mode of Action of Parathyroid Hormone and cyclic adenosine 3',5'-monophosphate on Renal Tubular Phosphate Reabsorption in the Dog. J. Clin. Invest. 1971;50:617-626.

doi:10.1172/JCI106532.Mode.

25. Collins JF, Bai L, Ghishan FK. The SLC20 family of proteins: dual functions as sodium-phosphate cotransporters and viral receptors. Pflugers Arch. 2004;447(5):647-52. doi:10.1007/s00424-003-1088-x.

26. Nishimura M, Naito S. Tissue-specific mRNA Expression Profiles of Human Solute Carrier Transporter Superfamilies. Drug Metab. Pharmacokinet. 2008;23(1):22-44.

27. Villa-Bellosta R, Ravera S, Sorribas V, et al. The Na+-Pi cotransporter PiT-2 (SLC20A2) is expressed in the apical membrane of rat renal proximal tubules and regulated by dietary Pi. Am. J. Physiol. Renal Physiol. 2009;296(4):F691-9. doi:10.1152/ajprenal.90623.2008.

28. Tenenhouse HS. Phosphate transport: molecular basis, regulation and pathophysiology. J. Steroid Biochem. Mol. Biol. 2007;103(3-5):572-7. doi:10.1016/j.jsbmb.2006.12.090.

29. Forster IC, Hernando N, Biber J, Murer H. Proximal tubular handling of phosphate: A molecular perspective. Kidney Int. 2006;70(9):1548-59. doi:10.1038/sj.ki.5001813.

30. Tenenhouse HS. Regulation of phosphorus homeostasis by the type iia na/phosphate cotransporter. Annu. Rev. Nutr. 2005;25:197-214. doi:10.1146/annurev.nutr.25.050304.092642.

31. Pfister MF, Hilfiker H, Forgo J, Lederer ED, Biber J, Murer H. Cellular mechanisms involved in the acute adaptation of $\mathrm{OK}$ cell $\mathrm{Na} / \mathrm{Pi}$-cotransport to highor low-Pi medium. Pflugers Arch. 1998;435(5):713-719. 
32. Weinman EJ, Steplock D, Shenolikar S, Blanpied TA. Dynamics of PTH-induced disassembly of Npt2a / NHERF-1 complexes in living OK cells. 2011:231-235. doi:10.1152/ajprenal.00532.2010.

33. Khan SR, Canales BK. Ultrastructural investigation of crystal deposits in Npt2a knockout mice: are they similar to human Randall's plaques? J. Urol. 2011;186(3):1107-13. doi:10.1016/j.juro.2011.04.109.

34. Beck L, Karaplis a C, Amizuka N, Hewson a S, Ozawa H, Tenenhouse HS. Targeted inactivation of $\mathrm{Npt} 2$ in mice leads to severe renal phosphate wasting, hypercalciuria, and skeletal abnormalities. Proc. Natl. Acad. Sci. U. S. A. 1998;95(9):5372-7. Available at:

http://www.pubmedcentral.nih.gov/articlerender.fcgi?artid=20268\&tool=pmcentre $\mathrm{z} \&$ rendertype $=$ abstract.

35. Iwaki T, Sandoval-Cooper MJ, Tenenhouse HS, Castellino FJ. A missense mutation in the sodium phosphate co-transporter Slc34a1 impairs phosphate homeostasis. J. Am. Soc. Nephrol. 2008;19(9):1753-62. doi:10.1681/ASN.2007121360.

36. Myakala Sarah Murer, Heini Wagner, Carsten A. Koesters, Robert Biber, Jürg Hernando, Nati KM. Renal-specific and inducible depletion of NaPi-IIc/Slc34a3, the cotransporter mutated in HHRH, does not affect phosphate or calcium homeostasis in mice. Am. J. Physiol. - Ren. Physiol. 2014;306(8):F833-F843. Available at: http://ajprenal.physiology.org/content/306/8/F833.abstract.

37. Murer H, Hernando N, Forster I, Biber J. Proximal tubular phosphate reabsorption: molecular mechanisms. Physiol. Rev. 2000;80(4):1373-409. Available at: http://www.ncbi.nlm.nih.gov/pubmed/11396609.

38. Stechman MJ, Loh NY, Thakker R V. Genetic causes of hypercalciuric nephrolithiasis. Pediatr. Nephrol. 2009;24(12):2321-32. doi:10.1007/s00467-0080807-0.

39. Tenenhouse HS. Phosphate transport: molecular basis, regulation and pathophysiology. J. Steroid Biochem. Mol. Biol. 2007;103(3-5):572-7. doi:10.1016/j.jsbmb.2006.12.090.

40. Prié D, Beck L, Friedlander G, Silve C. Sodium-phosphate cotransporters, nephrolithiasis and bone demineralization. Curr. Opin. Nephrol. Hypertens. 2004;13(6):675-81. Available at: http://www.ncbi.nlm.nih.gov/pubmed/15483460.

41. Magen D, Berger L, Coady M, et al. A Loss-of-Function Mutation in NaPi-IIa and Renal Fanconi’s Syndrome. N. Engl. J. Med. 2010;362:1102-1109. Available at: 
http://www.nejm.org/doi/full/10.1056/NEJMoa0905647. Accessed October 5, 2014.

42. Rajagopal a, Débora B, James TL, et al. Exome sequencing identifies a novel homozygous mutation in the phosphate transporter SLC34A1 in hypophosphatemia and nephrocalcinosis. J. Clin. Endocrinol. Metab. 2014;(October):jc20141517. doi:10.1210/jc.2014-1517.

43. Kenny J, Lees MM, Drury S, et al. Sotos syndrome, infantile hypercalcemia, and nephrocalcinosis: a contiguous gene syndrome. Pediatr. Nephrol. 2011;26(8):1331-4. doi:10.1007/s00467-011-1884-z.

44. Kronenberg HM. NPT2a - The Key to Phosphate Homeostasis. N. Engl. J. Med. 2002;347(13):1022-1024.

45. Weinman EJ, Lederer ED. NHERF-1 and the regulation of renal phosphate reabsoption: a tale of three hormones. Am. J. Physiol. Renal Physiol. 2012;303(3):F321-7. doi:10.1152/ajprenal.00093.2012.

46. Silver J, Naveh-Many T. Phosphate and the parathyroid. Kidney Int. 2009;75(9):898-905. doi:10.1038/ki.2008.642.

47. Bergwitz C, Jüppner H. Regulation of phosphate homeostasis by PTH, vitamin D, and FGF23. Annu. Rev. Med. 2010;61:91-104. doi:10.1146/annurev.med.051308.111339.

48. Caniggia A, Lore F, di Cairano G, Nuti R. Main endocrine modulators of vitamin D hydroxylases in human pathophysiology. J Steroid Biochem 1987;27(4-6):81524.

49. Wang W, Li C, Kwon T, et al. Reduced expression of renal Na+ transporters in rats with PTH-induced hypercalcemia. Am J Physiol Ren. Physiol 2004;286:534545.

50. Courbebaisse M, Leroy C, Bakouh N, et al. A new human NHERF1 mutation decreases renal phosphate transporter NPT2a expression by a PTH-independent mechanism. PLoS One 2012;7(4):e34764. doi:10.1371/journal.pone.0034764.

51. Guo J, Song L, Liu M, et al. Activation of a non-cAMP/PKA signaling pathway downstream of the PTH/PTHrP receptor is essential for a sustained hypophosphatemic response to PTH infusion in male mice. Endocrinology 2013;154(5):1680-9. doi:10.1210/en.2012-2240. 
52. Tomoe $\mathrm{Y}$, Segawa H, Shiozawa K, et al. Phosphaturic action of fibroblast growth factor 23 in Npt2 null mice. Am. J. Physiol. Renal Physiol. 2010;298(6):F1341-50. doi:10.1152/ajprenal.00375.2009.

53. Tenenhouse HS. Regulation of phosphorus homeostasis by the type iia na/phosphate cotransporter. Annu. Rev. Nutr. 2005;25:197-214. doi:10.1146/annurev.nutr.25.050304.092642.

54. Nitta K, Nagano N, Tsuchiya K. Fibroblast Growth Factor 23/Klotho Axis in Chronic Kidney Disease. Nephron. Clin. Pract. 2014;23(c). doi:10.1159/000365787.

55. Marsell R, Krajisnik T, Göransson H, et al. Gene expression analysis of kidneys from transgenic mice expressing fibroblast growth factor-23. Nephrol. Dial. Transplant 2008;23(3):827-33. doi:10.1093/ndt/gfm672.

56. Olauson H, Lindberg K, Amin R, et al. Targeted deletion of Klotho in kidney distal tubule disrupts mineral metabolism. J. Am. Soc. Nephrol. 2012;23(10):164151. doi:10.1681/ASN.2012010048.

57. Andrukhova O, Zeitz U, Goetz R, Mohammadi M, Lanske B, Erben RG. FGF23 acts directly on renal proximal tubules to induce phosphaturia through activation of the ERK1/2-SGK1 signaling pathway. Bone 2012;51(3):621-8. doi:10.1016/j.bone.2012.05.015.

58. Cunningham R, Biswas R, Brazie M, Steplock D, Shenolikar S, Weinman EJ. Signaling pathways utilized by PTH and dopamine to inhibit phosphate transport in mouse renal proximal tubule cells. Am. J. Physiol. Renal Physiol. 2009;296(2):F355-61. doi:10.1152/ajprenal.90426.2008.

59. Yamamoto H, Tani Y, Kobayashi K, et al. Alternative promoters and renal cellspecific regulation of the mouse type IIa sodium-dependent phosphate cotransporter gene. Biochim. Biophys. Acta 2005;1732(1-3):43-52. doi:10.1016/j.bbaexp.2005.11.003.

60. Silver J, Russell J, Sherwood LM. Regulation by vitamin D metabolites of messenger ribonucleic acid for preproparathyroid hormone in isolated bovine parathyroid cells. Proc. Natl. Acad. Sci. U. S. A. 1985;82(12):4270-3. Available at: http://www.pubmedcentral.nih.gov/articlerender.fcgi?artid=397979\&tool=pmcentr ez\&rendertype $=$ abstract.

61. Silver J, Yalcindag C, Sela-Brown A, Kilav R, Naveh-Many T. Regulation of the parathyroid hormone gene by vitamin $\mathrm{D}$, calcium and phosphate. Kidney Int. 
1999;73(Suppl. 73):S2-7. Available at:

http://www.ncbi.nlm.nih.gov/pubmed/10633456.

62. Barthel TK, Mathern DR, Whitfield GK, et al. 1,25-Dihydroxyvitamin D3/VDRmediated induction of FGF23 as well as transcriptional control of other bone anabolic and catabolic genes that orchestrate the regulation of phosphate and calcium mineral metabolism. J. Steroid Biochem. Mol. Biol. 2007;103(3-5):381-8. doi:10.1016/j.jsbmb.2006.12.054.

63. Friedlaender MM, Wald H, Dranitzki-Elhalel M, Zajicek HK, Levi M, Popovtzer MM. Vitamin D reduces renal NaPi-2 in PTH-infused rats: complexity of vitamin D action on renal Pi handling. Am J Physiol Ren. Physiol 2001;281:428-433.

64. Christov M, Koren S, Yuan Q, Baron R, Lanske B. Genetic ablation of sfrp4 in mice does not affect serum phosphate homeostasis. Endocrinology 2011;152(5):2031-6. doi:10.1210/en.2010-1351.

65. Sizova D, Velazquez H, Sampaio-Maia B, Quelhas-Santos J, Pestana M, Desir G V. Renalase regulates renal dopamine and phosphate metabolism. Am. J. Physiol. Renal Physiol. 2013;305(6):F839-44. doi:10.1152/ajprenal.00616.2012.

66. Wang M, Kurokawa K. Renal gluconeogenesis: axial and internephron heterogeneity and the effect of parathyroid hormone. Am J Physiol 1984;246(1):F59-66.

67. Kempson S, Kowalski J, Puschett J. Inhibition of renal brush border phosphate transport and stimulation of renal gluconeogenesis by cyclic AMP and parathyroid hormone. Biochem. Pharmacol. 1983;32(9):1533-1537.

68. Chobanian M, Hammerman M. Parathyroid hormone stimulates ammoniagenesis in canine renal proximal tubular segments. Am J Physiol 1988;255(5):F847-52. Available at: http://ajprenal.physiology.org/content/ajprenal/255/5/F847.full.pdf. Accessed October 6, 2014.

69. Puschett J, Zurbach P, Sylk D. Acute effects of parathyroid hormone on proximal bicarbonate transport in the dog. Kidney Int. 1976;9(6):501-510.

70. Jaeger P, Jones W, Kashgarian M, Segre G V, Hayslett J. Parathyroid hormone directly inhibits tubular reabsorption of bicarbonate in normocalcaemic rats with chronic hyperparathyroidism. Eur. J. Clin. Invest. 1987;17(5):415-420.

71. Khundmiri S, Lederer E. PTH and DA regulate Na-K ATPase through divergent pathways. Am J Physiol Ren. Physiol 2002;282:F512-522. Available at: 
http://ajprenal.physiology.org/content/282/3/F512.short. Accessed October 6, 2014.

72. Luigi P, Chiara FM, Laura Z, et al. Arterial Hypertension, Metabolic Syndrome and Subclinical Cardiovascular Organ Damage in Patients with Asymptomatic Primary Hyperparathyroidism before and after Parathyroidectomy: Preliminary Results. Int. J. Endocrinol. 2012;2012:408295. doi:10.1155/2012/408295.

73. Sprague SM, Moe SM. The case for routine parathyroid hormone monitoring. Clin. J. Am. Soc. Nephrol. 2013;8(2):313-8. doi:10.2215/CJN.04650512.

74. Hulter H, JC P. Acid-base homeostasis during chronic PTH excess in humans. Kidney Int. 1985;28(2):187-92.

75. Lind L, Hvarfner A, Palmer M, Grimelius L, Akerstrom G, Ljunghall S. Hypertension in primary hyperparathyroidism in relation to histopathology. Eur $J$ Surg 1991;157(8):457-9.

76. Weinman EJ, Steplock D, Wang Y, Shenolikar S. Characterization of a Protein Cofactor that Mediates Protein Kinase A Regulation of the Renal Brush Border Membrane Na+-H+ Exchanger. J. Clin. Invest. 1995;95(May):2143-2149. doi:10.1172/JCI117903.0.1.

77. Weinman EJ, Biswas RS, Peng G, et al. Parathyroid hormone inhibits renal phosphate transport by phosphorylation of serine 77 of sodium-hydrogen exchanger regulatory factor-1. J. Clin. Invest. 2007;117(11):3412-20. doi:10.1172/JCI32738.

78. Mahon MJ, Segre G V. Stimulation by parathyroid hormone of a NHERF-1assembled complex consisting of the parathyroid hormone I receptor, phospholipase Cbeta, and actin increases intracellular calcium in opossum kidney cells. J. Biol. Chem. 2004;279(22):23550-8. doi:10.1074/jbc.M313229200.

79. Hernando N, Deliot N, Gisler SM, et al. PDZ-domain interactions and apical expression of type IIa Na/Pi cotransporters. Proc. Natl. Acad. Sci. U. S. A. 2002;99(18):11957-11962.

80. Mahon MJ, Cole J a, Lederer ED, Segre G V. Na+/H+ exchanger-regulatory factor 1 mediates inhibition of phosphate transport by parathyroid hormone and second messengers by acting at multiple sites in opossum kidney cells. Mol. Endocrinol. 2003;17(11):2355-64. doi:10.1210/me.2003-0043.

81. Kempson S, Lötscher M, Kaissling B, Biber J, Murer H, Levi M. Parathyroid hormone action on phosphate transporter mRNA and protein in rat renal proximal 
tubules. Am. J. Physiol. 1995;268(4 Pt 2):F784-91. Available at: http://www.ncbi.nlm.nih.gov/pubmed/7733336.

82. Pfister MF, Ruf I, Stange G, et al. Parathyroid hormone leads to the lysosomal degradation of the renal type II Na/Pi cotransporter. Proc. Natl. Acad. Sci. U. S. A. 1998;95(4):1909-14. Available at:

http://www.pubmedcentral.nih.gov/articlerender.fcgi?artid=19212\&tool=pmcentre $\mathrm{z} \&$ rendertype=abstract.

83. Pfister MF, Lederer E, Forgo J, et al. Parathyroid Hormone-dependent Degradation of Type II Na+/Pi Cotransporters. J. Biol. Chem. 1997;272(32):20125-20130. doi:10.1074/jbc.272.32.20125.

84. Yang LE, Maunsbach AB, Leong PKK, McDonough A a. Differential traffic of proximal tubule $\mathrm{Na}+$ transporters during hypertension or PTH: NHE3 to base of microvilli vs. NaPi2 to endosomes. Am. J. Physiol. Renal Physiol. 2004;287(5):F896-906. doi:10.1152/ajprenal.00160.2004.

85. Murray RD, Holthouser K, Clark BJ, et al. Parathyroid hormone (PTH) decreases sodium-phosphate cotransporter type IIa (NpT2a) mRNA stability. Am. J. Physiol. Renal Physiol. 2013;304(8):F1076-85. doi:10.1152/ajprenal.00632.2012.

86. Collazo R, Fan L, Hu MC, Zhao H, Wiederkehr MR, Moe OW. Acute regulation of $\mathrm{Na}+\mathrm{H}+$ exchanger NHE3 by parathyroid hormone via NHE3 phosphorylation and dynamin-dependent endocytosis. J. Biol. Chem. 2000;275(41):31601-8. doi:10.1074/jbc.M000600200.

87. Gates F, Grant J. Experimental Observations on Irradiated, Normal, and Partially Parathyroidectomized Rabbits. J. Exp. Med. 1927;45(1):125-137.

88. Liu Y, Yang C, Li Z, et al. Safety, tolerability, pharmacokinetics, and pharmacodynamics of recombinant human parathyroid hormone (1-34) in healthy Chinese subjects. Clin. Ther. 2014;36(6):940-52. doi:10.1016/j.clinthera.2014.03.015.

89. Geer LY, Marchler-Bauer A, Geer RC, et al. The NCBI BioSystems database. Nucleic Acids Res. 2010;38(Database issue):D492-6. doi:10.1093/nar/gkp858.

90. Hartmann CM, Hewson a S, Kos $\mathrm{CH}$, et al. Structure of murine and human renal type II Na+-phosphate cotransporter genes (Npt2 and NPT2). Proc. Natl. Acad. Sci. U. S. A. 1996;93(14):7409-14. Available at: http://www.pubmedcentral.nih.gov/articlerender.fcgi?artid=38998\&tool=pmcentre $\mathrm{z} \&$ rendertype=abstract. 
91. Taketani Y, Miyamoto KI, Tanaka K, et al. Gene structure and functional analysis of the human Na+/phosphate co-transporter. Biochem. J. 1997;324 ( Pt 3:927-34. Available at:

http://www.pubmedcentral.nih.gov/articlerender.fcgi?artid=1218510\&tool=pmcen trez\&rendertype $=$ abstract.

92. Taketani Y, Segawa H, Chikamori M, et al. Regulation of Type II Renal Na+dependent Inorganic Phosphate Transporters by 1,25-Dihydroxyvitamin D3: IDENTIFICATION OF A VITAMIN D-RESPONSIVE ELEMENT IN THE HUMAN NAPI-3 GENE. J. Biol. Chem. 1998;273(23):14575-14581. doi:10.1074/jbc.273.23.14575.

93. Masuda M, Yamamoto H, Kozai M, et al. Regulation of renal sodium-dependent phosphate co-transporter genes (Npt2a and Npt2c) by all-trans-retinoic acid and its receptors. Biochem. J. 2010;429(3):583-92. doi:10.1042/BJ20100484.

94. Khundmiri SJ, Ahmad A, Bennett RE, et al. Novel regulatory function for NHERF-1 in Npt2a transcription. Am. J. Physiol. Renal Physiol. 2008;294(4):F840-9. doi:10.1152/ajprenal.00180.2007.

95. Ishiguro M, Yamamoto H, Masuda M, et al. Thyroid hormones regulate phosphate homoeostasis through transcriptional control of the renal type IIa sodiumdependent phosphate co-transporter (Npt2a) gene. Biochem. J. 2010;427(1):161-9. doi:10.1042/BJ20090671.

96. Biber RG, Jehle AW, Hilfiker H, et al. Type II Na-P i cotransport is regulated transcriptionally by ambient bicarbonate / carbon dioxide tension in OK cells. Am. J. Physiol. Ren. Physiol. 1999;276(1):F46-F53.

97. Xiao L, Naganawa T, Lorenzo J, Carpenter TO, Coffin JD, Hurley MM. Nuclear isoforms of fibroblast growth factor 2 are novel inducers of hypophosphatemia via modulation of FGF23 and KLOTHO. J. Biol. Chem. 2010;285(4):2834-46. doi:10.1074/jbc.M109.030577.

98. Levi M, Lötscher M, Sorribas V, et al. Cellular mechanisms of acute and chronic adaptation of rat renal $\mathrm{P}(\mathrm{i})$ transporter to alterations in dietary $\mathrm{P}(\mathrm{i})$. Am. J. Physiol. 1994;267(5 Pt 2):F900-8. Available at: http://www.ncbi.nlm.nih.gov/pubmed/7977794.

99. Tenenhouse HS, Martel J, Biber J, Murer H. Effect of P(i) restriction on renal $\mathrm{Na}(+)-\mathrm{P}(\mathrm{i})$ cotransporter mRNA and immunoreactive protein in X-linked Hyp mice. Am. J. Physiol. 1995;268(6 Pt 2):F1062-9. Available at: http://www.ncbi.nlm.nih.gov/pubmed/7611447. 
100. Werner a, Kempson S a, Biber J, Murer H. Increase of Na/Pi-cotransport encoding mRNA in response to low Pi diet in rat kidney cortex. J. Biol. Chem. 1994;269(9):6637-9. Available at: http://www.ncbi.nlm.nih.gov/pubmed/8120016.

101. Saxena S, Allon M. The Role of Cytosolic Calcium in Chronic Adaptation to Phosphate Depletion in Opossum Kidney Cells. J. Biol. Chem. 1996;271(7):39023906. doi:10.1074/jbc.271.7.3902.

102. Noronha-Blob L, Sacktor B. Inhibition by glucocorticoids of phosphate transport in primary cultured renal cells. J. Biol. Chem. 1986;261(5):2164-9. Available at: http://www.ncbi.nlm.nih.gov/pubmed/3944131.

103. Levi M, Shayman J a, Abe a, et al. Dexamethasone modulates rat renal brush border membrane phosphate transporter mRNA and protein abundance and glycosphingolipid composition. J. Clin. Invest. 1995;96(1):207-16. doi:10.1172/JCI118022.

104. Loffing J, Lötscher M, Kaissling B, et al. Renal Na/H exchanger NHE-3 and NaPO4 cotransporter NaPi-2 protein expression in glucocorticoid excess and deficient states. J. Am. Soc. Nephrol. 1998;9:1560-1567. Available at: http://jasn.asnjournals.org/content/9/9/1560.short. Accessed September 17, 2014.

105. Xu L, Dixit MP, Chen R, Dixit NM, Collins JF, Ghishan FK. Effects of angiotensin II on NaPi-IIa co-transporter expression and activity in rat renal cortex. Biochim. Biophys. Acta 2004;1667(2):114-21. doi:10.1016/j.bbamem.2004.09.005.

106. Ikeda S, Yamamoto H, Masuda M, et al. Downregulation of renal type IIa sodiumdependent phosphate cotransporter during lipopolysaccharide-induced acute inflammation. Am. J. Physiol. Renal Physiol. 2014;306(7):F744-50. doi:10.1152/ajprenal.00474.2013.

107. Kilav R, Silver J, Biber J, Murer H, Naveh-Many T. Coordinate regulation of rat renal parathyroid hormone receptor mRNA and Na-Pi cotransporter mRNA and protein. Am. J. Physiol. 1995;268(6 Pt 2):F1017-22. Available at: http://www.ncbi.nlm.nih.gov/pubmed/7611444.

108. Moe SM, Radcliffe JS, White KE, et al. The pathophysiology of early-stage chronic kidney disease-mineral bone disorder (CKD-MBD) and response to phosphate binders in the rat. J. Bone Miner. Res. 2011;26(11):2672-81. doi:10.1002/jbmr.485.

109. Hilfiker H, Hartmann CM, Stange G, Murer H. Characterization of the $5 \mathrm{~J}$ flanking region of OK cell type II Na-P i cotransporter gene. 1998;12(10):197-204. 
110. Lederer ED, Sohi SS, Mathiesen JM, Klein J. Regulation of expression of type II sodium-phosphate cotransporters by protein kinases A and C. Am J Physiol Ren. Physiol 1998;275(2):270-277.

111. Imanishi Y, Hosokawa Y, Yoshimoto K, et al. Primary hyperparathyroidism caused by parathyroid-targeted overexpression of cyclin D1 in transgenic mice. $J$. Clin. Invest. 2001;107(9):1093-102. doi:10.1172/JCI10523.

112. Mallya SM, Gallagher JJ, Wild YK, et al. Abnormal parathyroid cell proliferation precedes biochemical abnormalities in a mouse model of primary hyperparathyroidism. Mol. Endocrinol. 2005;19(10):2603-9. doi:10.1210/me.2005-0116.

113. Koyama H, Goodpasture C, Miller M, Teplitz R, Riggs A. Establishment and characterization of a cell line from the American opossum (Didelphys virginiana). In Vitro 1978;14(3):239-46.

114. Malström K, Stange G, Murer H. Identification of proximal tubular transport functions in the established kidney cell line, $\{\mathrm{OK}\}$. Biochim. Biophys. Acta Biomembr. 1987;902(2):269-277. doi:http://dx.doi.org/10.1016/00052736(87)90305-1.

115. Werner A, Moore ML, Mantei N, Biber J, Semenza G, Murer H. Cloning and expression of cDNA for a Na/Pi cotransport system of kidney cortex. Proc. Natl. Acad. Sci. U. S. A. 1991;88(21):9608-12. Available at: http://www.pubmedcentral.nih.gov/articlerender.fcgi?artid=52767\&tool=pmcentre $\mathrm{z} \&$ rendertype $=$ abstract.

116. Hilfiker H, Kvietikova I, Hartmann CM, Stange G, Murer H. Characterization of the human type II Na/Pi-cotransporter promoter. Pflugers Arch. 1998;436(4):5918. Available at: http://www.ncbi.nlm.nih.gov/pubmed/9683733.

117. Moz Y, Silver J, Naveh-Many T. Characterization of cis-acting element in renal NaPi-2 cotransporter mRNA that determines mRNA stability. Am. J. Physiol. Renal Physiol. 2003;284(4):F663-70. doi:10.1152/ajprenal.00332.2002.

118. Abou-Samra a B, Jüppner H, Force T, et al. Expression cloning of a common receptor for parathyroid hormone and parathyroid hormone-related peptide from rat osteoblast-like cells: a single receptor stimulates intracellular accumulation of both cAMP and inositol trisphosphates and increases intracel. Proc. Natl. Acad. Sci. U. S. A. 1992;89:2732-6. Available at: http://www.pubmedcentral.nih.gov/articlerender.fcgi?artid=48736\&tool=pmcentre $\mathrm{z} \&$ rendertype $=$ abstract. 
119. Watson PH, Fraher LJ, Hendy GN, et al. Nuclear localization of the type 1 PTH/PTHrP receptor in rat tissues. J. Bone Miner. Res. 2000;15(6):1033-44. doi:10.1359/jbmr.2000.15.6.1033.

120. Friedman PA, Gesek FA, Morley P, Whitfield JF, Willick GE. Cell-specific signaling and structure-activity relations of parathyroid hormone analogs in mouse kidney cells. Endocrinology 1999;140(1):301-309.

121. Watson PH, Fraher LJ, Hendy GN, et al. Nuclear localization of the type 1 PTH/PTHrP receptor in rat tissues. J. Bone Miner. Res. 2000;15(6):1033-44. doi:10.1359/jbmr.2000.15.6.1033.

122. Amizuka N, Lee HS, Kwan MY, et al. Cell-Specific Expression of the Parathyroid Hormone ( PTH )/ PTH-Related Peptide Receptor Gene in Kidney from KidneySpecific and Ubiquitous Promoters. Endocrinology 1997;138(1):469-481.

123. Nagai S, Okazaki M, Segawa H, et al. Acute down-regulation of sodiumdependent phosphate transporter NPT2a involves predominantly the cAMP/PKA pathway as revealed by signaling-selective parathyroid hormone analogs. J. Biol. Chem. 2011;286(2):1618-26. doi:10.1074/jbc.M110.198416.

124. Muff R, Fischer JA, Biber J, Murer H. Parathyroid Hormone Receptors in Control of Proximal Tubule Function. Annu. Rev. Physiol. 1992;54(1):67-79. doi:10.1146/annurev.ph.54.030192.000435.

125. Khundmiri SJ, Rane MJ, Lederer ED. Parathyroid hormone regulation of type II sodium-phosphate cotransporters is dependent on an A kinase anchoring protein. $J$. Biol. Chem. 2003;278(12):10134-41. doi:10.1074/jbc.M211775200.

126. Suarez F, Silve CM. Effect of Parathyroid Hormone on Arachidonic Acid Metabolism in Mouse Osteoblasts: Permissive Action of Dexamethasone. Endocrinology 1992;130(2):592-598.

127. Cole $\mathrm{J}$ a. Parathyroid hormone activates mitogen-activated protein kinase in opossum kidney cells. Endocrinology 1999;140(12):5771-9. doi:10.1210/endo.140.12.7173.

128. Yang S, Xiao L, Li J, Liu F, Sun L, Kanwar YS. Role of guanine-nucleotide exchange factor Epac in renal physiology and pathophysiology. Am. J. Physiol. Ren. Physiol. 2013;304(7):F831-9. doi:10.1152/ajprenal.00711.2012.

129. Li Y, Konings IBM, Zhao J, Price LS, de Heer E, Deen PMT. Renal expression of exchange protein directly activated by cAMP (Epac) 1 and 2. Am. J. Physiol. Ren. Physiol. 2008;295(2):F525-33. doi:10.1152/ajprenal.00448.2007. 
130. Ostrom RS, Bogard AS, Gros R, Feldman RD. Choreographing the adenylyl cyclase signalosome: sorting out the partners and the steps. Naunyn. Schmiedebergs. Arch. Pharmacol. 2012;385(1):5-12. doi:10.1007/s00210-0110696-9.

131. Bek MJ, Zheng S, Xu J, et al. Differential expression of adenylyl cyclases in the rat nephron. Kidney Int. 2001;60(3):890-9. doi:10.1046/j.15231755.2001.060003890.x.

132. Murtazina R, Kovbasnjuk O, Zachos NC, et al. Tissue-specific regulation of sodium/proton exchanger isoform 3 activity in $\mathrm{Na}(+) / \mathrm{H}(+)$ exchanger regulatory factor 1 (NHERF1) null mice. cAMP inhibition is differentially dependent on NHERF1 and exchange protein directly activated by cAMP in ileum versus . $J$. Biol. Chem. 2007;282(34):25141-51. doi:10.1074/jbc.M701910200.

133. Ferrandon S, Feinstein TN, Castro M, et al. Sustained cyclic AMP production by parathyroid hormone receptor endocytosis. Nat. Chem. Biol. 2009;5(10):734-42. doi:10.1038/nchembio.206.

134. Ahlström M, Lamberg-Allardt C. Rapid Protein Kinase A-Mediated Activation of Cyclic AMP-Phosphodiesterase by Parathyroid Hormone in UMR-106 Osteoblast-like Cells. J. Bone Miner. Res. 1997;12(2):172-178. Available at: http://onlinelibrary.wiley.com/doi/10.1359/jbmr.1997.12.2.172/full. Accessed October 8, 2014.

135. Whitfield JF, Isaacs RJ, Chakravarthy B, et al. Stimulation of protein kinase C activity in cells expressing human parathyroid hormone receptors by $\mathrm{C}$ - and $\mathrm{N}$ terminally truncated fragments of parathyroid hormone 1-34. J. Bone Miner. Res. 2001;16(3):441-7. doi:10.1359/jbmr.2001.16.3.441.

136. Mahon MJ, Donowitz M, Yun CC, Segre G V. Na+/H+ exchanger regulatory factor 2 directs parathyroid hormone 1 receptor signalling. Nature 2002;417(6891):858-861. Available at: http://dx.doi.org/10.1038/nature00816.

137. Wang B, Yang Y, Abou-Samra AB, Friedman P a. NHERF1 regulates parathyroid hormone receptor desensitization: interference with beta-arrestin binding. Mol. Pharmacol. 2009;75(5):1189-97. doi:10.1124/mol.108.054486.

138. Alonso V, Magyar CE, Wang B, Bisello A, Friedman P a. Ubiquitinationdeubiquitination balance dictates ligand-stimulated PTHR sorting. J. bone Miner. Res. 2011;26(12):2923-34. doi:10.1002/jbmr.494. 
139. Chauvin S, Bencsik M, Bambino T, Nissenson R a. Parathyroid hormone receptor recycling: role of receptor dephosphorylation and beta-arrestin. Mol. Endocrinol. 2002;16(12):2720-32. doi:10.1210/me.2002-0049.

140. Wang B, Bisello A, Yang Y, Romero GG, Friedman P a. NHERF1 regulates parathyroid hormone receptor membrane retention without affecting recycling. $J$. Biol. Chem. 2007;282(50):36214-22. doi:10.1074/jbc.M707263200.

141. Tawfeek H, Abou-Samra A. Important role for the V-type H+-ATPase and the Golgi apparatus in the recycling of PTH/PTHrP receptor. Am J Physiol Endocrinol Metab 2004;286:704-710. Available at: http://ajpendo.physiology.org/content/286/5/E704.short. Accessed October 11, 2014.

142. Pickard BW, Hodsman AB, Fraher LJ, Watson PH. Type 1 parathyroid hormone receptor (PTH1R) nuclear trafficking: regulation of PTH1R nuclear-cytoplasmic shuttling by importin-alpha/beta and chromosomal region maintenance 1/exportin 1. Endocrinology 2007;148(5):2282-9. doi:10.1210/en.2007-0157.

143. Silverstein DM, Spitzer A, Barac-Nieto M. Parathormone sensitivity and responses to protein kinases in subclones of opossum kidney cells. Pediatr. Nephrol. 2005;20(6):721-4. doi:10.1007/s00467-005-1832-x.

144. Cole J a, Eber SL, Poelling RE, Thorne PK, Forte LR. A dual mechanism for regulation of kidney phosphate transport by parathyroid hormone. Am. J. Physiol. 1987;253(2 Pt 1):E221-7. Available at: http://www.ncbi.nlm.nih.gov/pubmed/3618772.

145. Colet JA, Forte LR, Eber S, Thorne PK, Poelling RE. Regulation of SodiumDependent Phosphate Transport by Parathyroid Hormone in Opossum Kidney Cells : 2014;122(6).

146. Fenton R a, Murray F, Dominguez Rieg J a, Tang T, Levi M, Rieg T. Renal Phosphate Wasting in the Absence of Adenylyl Cyclase 6. J. Am. Soc. Nephrol. 2014;(9151):1-13. doi:10.1681/ASN.2013101102.

147. Weinstein LS, Yu S, Warner DR, Liu J. Endocrine manifestations of stimulatory G protein alpha-subunit mutations and the role of genomic imprinting. Endocr. Rev. 2001;22(5):675-705. doi:10.1210/edrv.22.5.0439.

148. Mantovani G. Clinical review: Pseudohypoparathyroidism: diagnosis and treatment. J. Clin. Endocrinol. Metab. 2011;96(10):3020-30. doi:10.1210/jc.20111048. 
149. Carpenter T, McPhee MD, Bort R, Mitnick MA, Carnes DL. Dissociation of phosphaturia and 25(OH)D-1a-hydroxylase trophism using a novel analogue of parathyroid hormone. Am. J. Physiol. 1992;25:483-487.

150. Ranch D, Zhang MY, Portale A a, Perwad F. Fibroblast growth factor 23 regulates renal 1,25-dihydroxyvitamin D and phosphate metabolism via the MAP kinase signaling pathway in Hyp mice. J. Bone Miner. Res. 2011;26(8):1883-90. doi:10.1002/jbmr.401.

151. Jouishomme H, Whitfield JF, Gagnon L, et al. Further definition of the protein kinase $\mathrm{C}$ activation domain of the parathyroid hormone. J. Bone Miner. Res. 1994;9(6):943-949. doi:10.1002/jbmr.5650090620.

152. Whitfield J, Isaacs R, Chakravarthy B, et al. Protein Kinase C Activity in Cells Expressing Human Parathyroid Hormone Receptors by $\mathrm{C}$-and $\mathrm{N}$-Terminally Truncated Fragments of Parathyroid Hormone 1. J. Bone Miner. Res. 2001;16(3):441-447. Available at: http://onlinelibrary.wiley.com/doi/10.1359/jbmr.2001.16.3.441/full. Accessed September 4, 2014.

153. Phelps E, Bezouglaia O, Tetradis S, Nervina JM. Parathyroid hormone induces receptor activity modifying protein-3 (RAMP3) expression primarily via 3',5'cyclic adenosine monophosphate signaling in osteoblasts. Calcif. Tissue Int. 2005;77(2):96-103. doi:10.1007/s00223-004-0239-1.

154. Enserink JM, Christensen AE, de Rooij J, et al. A novel Epac-specific cAMP analogue demonstrates independent regulation of Rap1 and ERK. Nat. Cell Biol. 2002;4(11):901-6. doi:10.1038/ncb874.

155. Dutta D, Donaldson JG. Search for inhibitors of endocytosis: Intended specificity and unintended consequences. Cell. Logist. 2012;2(4):203-208.

156. Segawa H, Yamanaka S, Onitsuka A, et al. Parathyroid hormone-dependent endocytosis of renal type IIc Na-Pi cotransporter. Am. J. Physiol. Renal Physiol. 2007;292(1):F395-403. doi:10.1152/ajprenal.00100.2006.

157. Yu P, Han W, Villar VAM, et al. Dopamine D1 receptor-mediated inhibition of NADPH oxidase activity in human kidney cells occurs via protein kinase Aprotein kinase C cross talk. Free Radic. Biol. Med. 2011;50(7):832-40. doi:10.1016/j.freeradbiomed.2010.12.027.

158. Azarani A, Goltzman D, Orlowski J. Parathyroid hormone and parathyroid hormone-related peptide inhibit the apical $\mathrm{Na}+\mathrm{H}+$ exchanger NHE-3 isoform in 
renal cells $(\mathrm{OK})$ via a dual signaling cascade involving protein kinase A and C. $J$. Biol. Chem. 1995;270(34):20004-20010.

159. Honegger K, Capuano P, Winter C, et al. Regulation of sodium-proton exchanger isoform 3 (NHE3) by PKA and exchange protein directly activated by cAMP (EPAC). Proc. Natl. Acad. Sci. U. S. A. 2006;103(11):803-808. Available at: http://www.pnas.org/content/103/3/803.short. Accessed September 4, 2014.

160. Queiroz-Leite GD, Peruzzetto MC, Neri E a, Rebouças N a. Transcriptional regulation of the $\mathrm{Na}^{+} / \mathrm{H}^{+}$exchanger NHE3 by chronic exposure to angiotensin II in renal epithelial cells. Biochem. Biophys. Res. Commun. 2011;409(3):470-6. doi:10.1016/j.bbrc.2011.05.028.

161. Ghosh S, Jacobson A. RNA decay modulates gene expression and controls its fidelity. Wiley Interdiscip. Rev. RNA 2010;1(3):351-361. doi:10.1002/wrna.25.RNA.

162. Andreassi C, Riccio A. To localize or not to localize: mRNA fate is in 3 'UTR ends. Trends Cell Biol. 2009;19(9):465-74. doi:10.1016/j.tcb.2009.06.001.

163. Wang ET, Sandberg R, Luo S, et al. Alternative isoform regulation in human tissue transcriptomes. Nature 2008;456(7221):470-6. doi:10.1038/nature07509.

164. Chen C-YA, Shyu A-B. AU-rich elements: characterization and importance in mRNA degradation. Trends Biochem. Sci. 1995;20(11):465-470. doi:http://dx.doi.org/10.1016/S0968-0004(00)89102-1.

165. Peng SS, Chen CA, Shyu A-B. Functional characterization of a non-AUUUA AUrich element from the c-jun proto-oncogene mRNA : evidence for a novel class of AU-rich elements. Mol. Cell. Biol. 1996;16(4):1490-1499.

166. Halees AS, El-Badrawi R, Khabar KS a. ARED Organism: expansion of ARED reveals AU-rich element cluster variations between human and mouse. Nucleic Acids Res. 2008;36(Database issue):D137-40. doi:10.1093/nar/gkm959.

167. White EJF, Brewer G, Wilson GM. Post-transcriptional control of gene expression by AUF1: Mechanisms, physiological targets, and regulation. Biochim. Biophys. Acta - Gene Regul. Mech. 2013;1829(6-7):680-688. doi:http://dx.doi.org/10.1016/j.bbagrm.2012.12.002.

168. Moz Y. Protein-RNA Interactions Determine the Stability of the Renal NaPi-2 Cotransporter mRNA and Its Translation in Hypophosphatemic Rats. J. Biol. Chem. 1999;274(36):25266-25272. doi:10.1074/jbc.274.36.25266. 
169. Reshkin S, Wuarin F, Biber J, Murer H. Parathyroid Hormone-induced Alterations of Protein Content and Phosphorylation in Enriched Apical Membranes of Opossum Kidney Cells. J. Biol. Chem. 1990;265(25):15261-15266.

170. Gruber AR, Lorenz R, Bernhart SH, Neuböck R, Hofacker IL. The Vienna RNA websuite. Nucleic Acids Res. 2008;36(Web Server issue):W70-4. doi:10.1093/nar/gkn188.

171. Lorenz R, Bernhart SH, Höner Zu Siederdissen C, et al. ViennaRNA Package 2.0. Algorithms Mol. Biol. 2011;6:26. doi:10.1186/1748-7188-6-26.

172. Cook KB, Kazan H, Zuberi K, Morris Q, Hughes TR. RBPDB: a database of RNA-binding specificities. Nucleic Acids Res. 2011;39(Database issue):D301-8. doi:10.1093/nar/gkq1069.

173. Merchant ML. Mass Spectrometry in Chronic Kidney Disease Research. Adv. Chronic Kidney Dis. 2014;17(6):455-468. doi:10.1053/j.ackd.2010.09.003.

174. Consortium TU. Activities at the Universal Protein Resource (UniProt). Nucleic Acids Res. 2014;42(Database issue):D191-8. doi:10.1093/nar/gkt1140.

175. Huang DW, Sherman BT, Lempicki R a. Bioinformatics enrichment tools: paths toward the comprehensive functional analysis of large gene lists. Nucleic Acids Res. 2009;37(1):1-13. doi:10.1093/nar/gkn923.

176. Huang DW, Sherman BT, Lempicki R a. Systematic and integrative analysis of large gene lists using DAVID bioinformatics resources. Nat. Protoc. 2009;4(1):4457. doi:10.1038/nprot.2008.211.

177. Flicek P, Amode MR, Barrell D, et al. Ensembl 2014. Nucleic Acids Res. 2014;42(Database issue):D749-55. doi:10.1093/nar/gkt1196.

178. Muppirala UK, Lewis BA, Dobbs D. Computational Tools for Investigating RNAProtein Interaction Partners. J. Comput. Sci. Syst. Biol. 2013;06(04):182-187. doi:10.4172/jcsb.1000115.

179. Muppirala UK, Honavar VG, Dobbs D. Predicting RNA-protein interactions using only sequence information. BMC Bioinformatics 2011;12(1):489. doi:10.1186/1471-2105-12-489.

180. Utz VM, Beight CD, Marino MJ, Hagstrom SA, Traboulsi EI. Autosomal Dominant Retinitis Pigmentosa Secondary to Pre-mRNA Splicing-Factor Gene PRPF31 (RP11): Review of Disease Mechanism and Report of a Family with a Novel 3-Base Pair Insertion. Ophthalmic Genet. 2013;34(4):183-188. doi:10.3109/13816810.2012.762932. 
181. Leppek K, Schott J, Reitter S, Poetz F, Hammond MC, Stoecklin G. Roquin promotes constitutive mRNA decay via a conserved class of stem-loop recognition motifs. Cell 2013;153(4):869-81. doi:10.1016/j.cell.2013.04.016.

182. Nechama M, Ben-Dov IZ, Briata P, Gherzi R, Naveh-Many T. The mRNA decay promoting factor $\mathrm{K}$-homology splicing regulator protein post-transcriptionally determines parathyroid hormone mRNA levels. FASEB J. 2008;22(10):3458-68. doi:10.1096/fj.08-107250.

183. Briata P, Chen C-Y, Ramos A, Gherzi R. Functional and molecular insights into KSRP function in mRNA decay. Biochim. Biophys. Acta 2013;1829(6-7):689-94. doi:10.1016/j.bbagrm.2012.11.003.

184. Eliscovich C, Buxbaum AR, Katz ZB, Singer RH. mRNA on the move: the road to its biological destiny. J. Biol. Chem. 2013;288(28):20361-8. doi:10.1074/jbc.R113.452094.

185. Chen C-Y, Gherzi R, Ong S-E, et al. AU Binding Proteins Recruit the Exosome to Degrade ARE-Containing mRNAs. Cell 2001;107(4):451-464. doi:10.1016/S0092-8674(01)00578-5.

186. Gherzi R, Lee K-Y, Briata P, et al. A KH Domain RNA Binding Protein, KSRP, Promotes ARE-Directed mRNA Turnover by Recruiting the Degradation Machinery. Mol. Cell 2014;14(5):571-583. doi:10.1016/j.molcel.2004.05.002.

187. Chou C, Mulky A, Maitra S, et al. Tethering KSRP , a Decay-Promoting AU-Rich Element-Binding Protein , to mRNAs Elicits mRNA Decay. Mol. Cell. Biol. 2006;26(10):3695-3706. doi:10.1128/MCB.26.10.3695.

188. Bertossi A, Aichinger M, Sansonetti P, et al. Loss of Roquin induces early death and immune deregulation but not autoimmunity. J. Exp. Med. 2011;208(9):174956. doi:10.1084/jem.20110578.

189. Vogel KU, Edelmann SL, Jeltsch KM, et al. Roquin paralogs 1 and 2 redundantly repress the Icos and Ox40 costimulator mRNAs and control follicular helper T cell differentiation. Immunity 2013;38(4):655-68. doi:10.1016/j.immuni.2012.12.004.

190. Tan D, Zhou M, Kiledjian M, Tong L. The ROQ domain of Roquin recognizes mRNA constitutive-decay element and double-stranded RNA. Nat. Struct. Mol. Biol. 2014;21(8):679-85. doi:10.1038/nsmb.2857.

191. Schlundt A, Heinz G a, Janowski R, et al. Structural basis for RNA recognition in roquin-mediated post-transcriptional gene regulation. Nat. Struct. Mol. Biol. 2014;21(8):671-8. doi:10.1038/nsmb.2855. 
192. Quarles K a, Sahu D, Havens M a, et al. Ensemble analysis of primary microRNA structure reveals an extensive capacity to deform near the Drosha cleavage site. Biochemistry 2013;52(5):795-807. doi:10.1021/bi301452a.

193. Briata $\mathrm{P}$, Chen C-Y, Giovarelli M, et al. KSRP, many functions for a single protein. Front. Biosci. 2011;16:1787-1796.

194. Díaz-Moreno I, Hollingworth D, Frenkiel T a, et al. Phosphorylation-mediated unfolding of a KH domain regulates KSRP localization via 14-3-3 binding. Nat. Struct. Mol. Biol. 2009;16(3):238-46. doi:10.1038/nsmb.1558.

195. Gherzi R, Trabucchi M, Ponassi M, et al. The RNA-binding protein KSRP promotes decay of beta-catenin mRNA and is inactivated by PI3K-AKT signaling. PLoS Biol. 2006;5(1):e5. doi:10.1371/journal.pbio.0050005.

196. Obenauer JC, Cantley LC, Yaffe MB. Scansite 2.0: proteome-wide prediction of cell signaling interactions using short sequence motifs. Nucleic Acids Res. 2003;31(13):3635-3641. doi:10.1093/nar/gkg584. 


\section{APPENDIX}

\section{$\underline{\text { Proteins }}$}

KHSRP - hnRNPK-homology (KH)-type Splicing Regulatory Protein

$\mathrm{Na}^{+} / \mathrm{K}^{+}$-ATPase - sodium potassium ATPase

NBC1 - sodium-bicarbonate cotransporter isoform 1

NHE3 - sodium-hydrogen exchanger isoform 3

NHERF-1 - sodium-hydrogen exchanger regulatory factor isoform 1

Npt2a - type IIa sodium-phosphate cotransporter

$\mathrm{Npt} 2 \mathrm{c}$ - type IIc sodium-phosphate cotransporter

PiT-2 - inorganic phosphate transporter isoform 2

PTHR1 - PTH receptor type I

RC3H2 - Roquin-2

ZFP36L1 - Zinc Finger Protein 36, C3H1 Type-Like 1

\section{Cell signaling}

AC - adenylyl cyclase

AKAP79/150 - A kinase anchor protein

$\mathrm{Akt}$ - serine/threonine protein kinase; also referred to as Protein Kinase B

cAMP - cyclic adenosine monophosphate

Epac - exchange protein activated by cAMP

ERK1/2 - extracellular-signal-regulated kinase

FGF23 - fibroblast growth factor isoform 23

GPCR - G-protein-coupled receptor

$\mathrm{IP}_{3}$ - inositol triphosphoate 
MAPK - mitogen-activated protein kinase

PDE - phosphodiesterase

PKA - protein kinase A

$\mathrm{PKC}$ - protein kinase $\mathrm{C}$

PLC - phospholipase C

PTH - parathyroid hormone

PTHrP - PTH-related peptide

\section{$\underline{\text { Molecular biology }}$}

RBP - RNA-binding protein

$\mathrm{CDE}$ - constitutive decay element

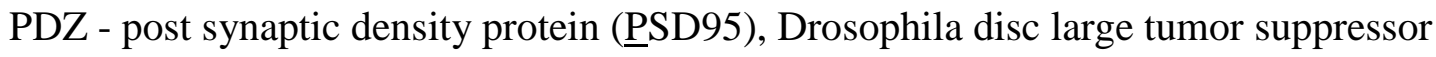
(Dlg1), and zonula occludens-1 protein (Z्Z-1)

UTR - untranslated region mRNP - ribonucleoprotein complex composed of mRNA and associated RBPs

$\mathrm{PABP}-\operatorname{poly}(\mathrm{A})$ binding protein

eIF4G - eukaryotic translation initiation factor 4 gamma

eIF4E - eukaryotic translation initiation factor $4 \mathrm{E}$

ncRNA - non-coding RNA

siRNA - small interfering RNA

miRNA - micro RNA

RISC - RNA-induced silencing complex

ARE - AU-rich element

ARE-BP - AU-rich element binding protein

$\mathrm{HuR}$ - Human antigen $\mathrm{R}$

ELAVL1 - embryonic lethal abnormal vision-like 1; synonym for HuR

RIP - RNA-binding protein immunoprecipitation

IP - immunoprecipitation

eIF4A1 - eukaryotic translation initiation factor 4A1 


\section{$\underline{\text { Methods \& Reagents }}$}

8-Br-cAMP - 8-bromo-cyclic adenosine monophosphate

Act D - actinomycin D

ANOVA - analysis of variance

$\mathrm{BBM}$ - brush-border membrane

BLM - basolateral membrane

BSA - bovine serum albumin

Chx - cycloheximide

$\mathrm{CM}$ - crude membrane

$\mathrm{CPM}$ - counts per minute

$\mathrm{CPZ}$ - chlorpromazine

DAB - 3,3'-Diaminobenzidine

DAG - diacylglycerol

DAVID - The Database for Annotation, Visualization and Integrated Discovery

FVB - background strain of transgenic PTH-D1 mice

H-89 - pharmacologic inhibitor of PKA

HRP - horse radish peroxidase

IHC - immunohistochemistry

OK - opossum kidney

$\mathrm{PKCi}$ - PKC inhibitor peptide

PMA - phorbol myristate acetate

PTH-D1 - transgenic mouse strain featuring parathyroid-specific cyclin D1 overexpression

RF - Random Forest

RPISeq - RNA-Protein Interaction Prediction

RT-qPCR - reverse transcription quantitative polymerase chain reaction

SDS-PAGE - sodium dodecyl sulfate polyacrylamide gel electrophoresis

SVM - Support vector machine

TTBS - Tween-tris-buffered saline 


\section{CURRICULUM VITAE Rebecca Murray, Ph.D.}

Department of Physiology and Biophysics

University of Louisville, Louisville, KY

(812) 493 - 9794

rebecca.murray@louisville.edu

\section{EDUCATION}

UNIVERSITY OF LOUISVILLE - LOUISVILLE, KY

M.S. in Physiology \& Biophysics earned December 2013

Ph.D. in Physiology \& Biophysics earned December 2014

Cumulative GPA 4.0

NORTHWESTERN UNIVERSITY - EVANSTON, IL

B.A. in Psychology

Cumulative GPA 3.587

\section{HONORS AND AWARDS}

- 2012 - 2013 University of Louisville Faculty Favorite

- Student-nominated award for outstanding professorship.

- Caroline tum Suden/Frances Hellebrandt Professional Opportunity Award, American Physiological Society

- Travel funding to the 2013 Experimental Biology meeting in Boston, MA.

- American Physiological Society Epithelial Transport Group travel award

- Travel funding to attend the 2013 Experimental Biology meeting in Boston, MA.

- Research!Louisville $2012,3^{\text {rd }}$ place

○ Master's graduate student division. 


\section{SKILLS ACQUIRED}

Molecular biology techniques: $\quad$ Cell culture:

Light microscopy

Confocal microscopy

Immunohistochemistry

Immunoprecipitation

Western blotting

Reverse transcription

Quantitative PCR

Radioactivity assays

Luciferase reporter assays
Plasmid transfection

Aseptic techniques

Instrumentation:

Biotek Synergy 2 plate reader

Applied Biosystems 7500 Real Time PCR System Olympus Fluoview FV1000 confocal microscope Syngene PXi 6 multi-application imaging system

Processing techniques:

Membrane preparation from tissue and cell culture

Sucrose density gradient centrifugation for organelle isolation

Plasmid cloning, enzyme restriction

Bacterial transformation and plasmid isolation

RNA isolation from tissue and cell culture

Tissue paraffin block sectioning

\section{TEACHING EXPERIENCE}

\begin{tabular}{llll}
\hline Date & Department & Course & Description \\
\hline $\begin{array}{l}\text { July 2012 } \\
\text { July 2014 }\end{array}$ & Medicine & $\begin{array}{l}\text { Pre-matriculation summer } \\
\text { program, Physiology } \\
\text { section }\end{array}$ & Lecturer \\
$\begin{array}{l}\text { Spring 2013- } \\
\text { present }\end{array}$ & Medicine & $\begin{array}{l}\text { PHZB 801, Medical } \\
\text { Physiology }\end{array}$ & Tutor \\
$\begin{array}{l}\text { Spring 2013 } \\
\text { Spring 2015 }\end{array}$ & $\begin{array}{l}\text { Physiology \& } \\
\text { Biophysics }\end{array}$ & $\begin{array}{l}\text { PHZB 609, Integrated } \\
\text { Systemic Physiology }\end{array}$ & Facilitator \\
$\begin{array}{l}\text { January 2013 }- \\
\text { present }\end{array}$ & Dentistry & BMSC 804, Biochemistry & Tutor \\
$\begin{array}{l}\text { September 2013 }- \\
\text { present }\end{array}$ & Dentistry & $\begin{array}{l}\text { BMSC 805, Systemic } \\
\text { Physiology }\end{array}$ & Tutor \\
\hline
\end{tabular}




\section{JOURNAL ARTICLES}

Murray RD, Holthouser K, Clark B, Salyer S, Barati MT, Khundmiri SJ, Lederer ED. "Parathyroid hormone decreases sodium-phosphate type IIa (NpT2a) mRNA stability." Am J Physiol Renal Physiol 304: F1076-F1085, 2013.

Khundmiri SJ, Salyer SA, Farmer B, Qipshidze N, Murray RD, Clark BJ, Xie Z, Pressley TA, Lederer ED. "Structural determinants for the ouabain-stimulated increase in Na-K ATPase activity." Biochimica et Biophysica Acta - Molecular Cell Research 1843(6): 1089-1102, 2014.

Khundmiri SJ, Murray RD, Lederer ED. "PTH and Vitamin D." Submitted to Comprehensive Physiology, October 2014.

Lederer ED, Khundmiri SJ, Clark BJ, Murray RD, Weinman EJ. "Role of the sodium hydrogen exchanger regulatory factor 1 (NHERF1) in forward trafficking of the type IIa sodium phosphate cotransporter (Npt2a)." In submission. December 2014.

\section{ORAL PRESENTATIONS}

- "Phosphate: It's what's for dinner."

○ University of Louisville Physiology \& Biophysics seminar series. September 16, 2014.

- "The fate of phosphate in the proximal tubule."

○ UofL Kidney Disease Program research conference. February 7, 2014.

- "Signaling mechanisms in post-transcriptional regulation of type IIa sodiumphosphate cotransporter mRNA by parathyroid hormone."

- Southern Salt and Water Kidney Club annual meeting, Sarasota, FL. December 6, 2013.

- University of Louisville Physiology \& Biophysics seminar series. September 17, 2013.

- "Post-transcriptional regulation of type IIa sodium-phosphate cotransporter mRNA by parathyroid hormone."

- Lake Cumberland Biological Transport Group annual meeting, Lake Cumberland, KY. June 16, 2013.

- UofL Kidney Disease Program research conference. May 31, 2013. 
- "Parathyroid hormone decreases mRNA stability of the Type IIa sodiumphosphate cotransporter."

- Epithelial Transport Group session at Experimental Biology, Boston, MA. April 20, 2013.

- "Post-transcriptional regulation of renal proximal tubule phosphate reabsorption by parathyroid hormone."

- Invited presentation, University of Louisville Bioinformatics Journal Club. November 14, 2012.

- "PTH and renal phosphate transport: Decoding the message."

- Lake Cumberland Biological Transport Group annual meeting, Lake Cumberland, KY. June 19, 2012.

- "Regulation of renal proximal tubule phosphate reabsorption in response to chronic hyperparathyroidism."

○ University of Louisville Physiology \& Biophysics seminar series. March 6, 2012.

\section{POSTER PRESENTATIONS}

- "Characterization of the PTH-responsive phosphoproteome in the proximal tubule"

- American Society of Nephrology, Kidney Week 2014, Philadelphia, PA. November 14, 2014.

- 2014 Midwest Membrane Trafficking and Signaling Symposium, Louisville, KY. October 3, 2014.

- Research!Louisville annual symposium, Louisville, KY. September 16, 2014.

- "Signaling mechanisms for post-transcriptional regulation of the type IIa sodiumphosphate cotransporter by parathyroid hormone"

- Experimental Biology annual meeting, San Diego, CA. April 28, 2014.

- Kentucky Physiological Society annual meeting, Louisville, KY. March 31, 2014.

- "Parathyroid hormone inhibits type IIa sodium phosphate cotransporter mRNA expression through both protein kinase A- and protein kinase C-dependent mechanisms."

- American Society of Nephrology, Kidney Week, Atlanta, GA, November 4, 2013.

- Research!Louisville annual symposium, Louisville, KY. September 10, 2013. 
- "Parathyroid hormone decreases mRNA stability of the type IIa sodiumphosphate cotransporter."

○ Experimental Biology annual meeting, Boston, MA. April 24, 2013.

○ Kentucky Physiological Society annual meeting, Lexington, KY. March 25, 2013.

- "The type IIa sodium-phosphate cotransporter is transcriptionally regulation by parathyroid hormone."

○ American Society of Nephrology, Kidney Week 2012, San Diego, CA. November 3, 2012.

- Research!Louisville annual symposium, Louisville, KY. September 18, 2012.

- "Renal proximal tubule ion transporters are discrepantly regulated by parathyroid hormone in acute versus chronic hyperparathyroidism."

○ Experimental Biology, San Diego, CA. April 23, 2012.

- "Parathyroid hormone differentially regulates the expression of proximal tubule ion transporters in acute versus chronic hyperparathyroidism."

○ Research!Louisville annual symposium, Louisville, KY. October 11, 2011.

\section{LEADERSHIP POSITIONS}

- Session chair, Lake Cumberland Biology Transport Group

○ "Effect of carbon nanotubes on gramicidin channels in artificial lipid membranes"

○ Lake Cumberland, KY, June 9, 2014

- Graduate student panel, Department of Physiology and Biophysics

○ "Finding a Mentor"

- Served on a panel of senior graduate students on selecting a mentor for beginning master's students

- September 2013

- Graduate Student Council, University of Louisville

○ 2013 term

- Served as department representative

- Physiology department student president, University of Louisville

○ 2014 term

- Served as department representative for graduate student affairs 


\section{COMMUNITY INVOLVEMENT}

- Ballard High School Science Fair - judge

$2012-2014$

- Louisville Regional Science Fair - judge

$2013-2014$

- PhUn Week (Physiology Understanding)

October 2013

- St. Bernard School, Louisville, KY

- Outreach program sponsored by the American Physiological Society

- Worked with $8^{\text {th }}$ grade students on their presentations about cardiovascular health and healthy living

- DuPont Manual High School Science Olympiad - judge

March 2012

○ Created, administered, and scored "Food Science" and "Disease Detectives" tests for middle and high school categories

\section{PAST AND CURRENT TRAINEES}

\section{$\underline{\text { Master's students }}$}

- Clayton Connor

- Previous Physiology \& Biophysics master's student

- Current $1^{\text {st }}$ year medical student at University of Louisville

○ November 2013 - July 2014

- "Ouabain-induced association between Na-K ATPase and $A_{1} T_{1}$ "

- Corey Ketchem

○ Physiology \& Biophysics master's student

○ Matriculating into University of Louisville School of Medicine July 2015

- November 2013 - present

- "Role of NHERF-1 in the forward trafficking of Npt2a"

\section{$\underline{\text { Undergraduate students }}$}

- Lauren Grant

- Current senior at the University of Louisville

- Summer 2013 (Rising junior)

- "Role of NHERF-1 in proximal tubule protein expression"

- Co-mentor during Summer Research Opportunity Program 
- Madelyn DuPlessis

○ Previous undergraduate student at Centre College, Danville, KY

- Current $1^{\text {st }}$ year dental student at University of Kentucky School of Dentistry

- Summer 2013 (Rising senior)

- "Ouabain-induced association between Na-K ATPase and $A_{1} T_{1}$ "

- Co-mentor during Kentucky Biomedicial Research Infrastructure Network (KBRIN) program

- Brandon Farmer

○ Current junior at Western Kentucky University, Bowling Green, KY.

- Summer 2012 (Rising freshman)

- "Ouabain-stimulated associated between Na-K-ATPase and NHE1: A TIRF and FRET analysis."

- Co-mentor during KBRIN summer program

$\underline{\text { High school students }}$

- Steven St. Clair

- Current senior at Ballard High School, Louisville, KY

- August 2013 - January 2014

- Science fair project co-mentor

- "Cancer cell metastasis: Role of Vacuolar ATPase"

- Emmanuel Flemister

- Current senior at Ballard High School, Louisville, KY

- Science fair project co-mentor

○ 2012 - 2013 (sophomore year)

- "Cancer cells: A tale of two proteins."

○ 2011-2012 (freshman year)

- "Environmental effects on bacterial growth." 